\title{
12. TRACE-ELEMENT GEOCHEMISTRY OF VOLCANIC ROCKS FROM SITE 786: THE IZU-BONIN FOREARC ${ }^{1}$
}

\author{
Bramley J. Murton, ${ }^{2}$ David W. Peate, ${ }^{3}$ Richard J. Arculus, ${ }^{4}$ Julian A. Pearce, ${ }^{3}$ and Sieger van der Laan ${ }^{5}$
}

\begin{abstract}
Eocene-Oligocene volcanic rocks drilled at Site 786 in the Izu-Bonin forearc cover a wide range of compositions from primitive boninites to highly evolved rhyolites. K-Ar dating reveals at least two distinct episodes of magmatism; one at $41 \mathrm{Ma}$ and a later one at $35 \mathrm{Ma}$. The early episode produced low-Ca boninites and bronzite andesites that form an oceanic basement of pillow lavas and composite intrusive sheets, overlain by flows and intrusive sheets of intermediate-Ca boninites and bronzite-andesites and a fractionated series of andesites, dacites, and rhyolites. The later episode produced high-Ca boninites and intermediate-Ca boninites, exclusively as intrusive sheets.
\end{abstract}

Trace element data indicate that all of the evolved chemical groups at Site 786 can be related by fractionation and/or accumulation of the observed mineral phases back to the three boninite groups, which represent distinct parental magmas. The boninites have very low abundances of $\mathrm{Ti}, \mathrm{Y}$, and $\mathrm{HREE}$ relative to MORB, consistent with an origin from a depleted source, and consideration of $\mathrm{Cr}$-Y-Ti melting systematics and major element data indicates that the low-Ca boninites came from a source more depleted than the high-Ca boninite source. The boninites show enrichment in LIL elements, LREE and selected HFS ( $\mathrm{Zr}, \mathrm{Hf}$ ) relative to $\mathrm{Ti}, \mathrm{Y}$, HREE which reflect the addition of a "subduction" component to the boninite source region. The distinctive enrichment of $\mathrm{Zr}$ is a feature not found in typical arc-related volcanics, but it has been recognized in several other boninite suites. The fractionation of $\mathrm{Zr}$ from $\mathrm{Sm}$ and Ti suggests an important role for amphibole in any petrogenetic model to explain the genesis of these boninites. Possibilities include the addition of a melt derived from subducted amphibolitized ocean crust and the interaction of an OIB-like melt with amphibole stabilized in the mantle wedge.

The multiple episodes of boninite magmatism at Site 786 imply a recurrence of conditions for boninite genesis over an extended period of time (at least 7 m.y.). This refutes the idea that boninites are generated solely at the initiation of subduction. However, the predominance of LREE and $\mathrm{Zr}, \mathrm{Ta}, \mathrm{Nb}$ enrichment in the Eocene boninite groups does imply a genetic relationship with the onset of subduction and may be explained by early hydrous melting of amphibolitized forearc lithospheric mantle combined with mobilization of a pre-existing OIB-like component.

\section{INTRODUCTION}

The Izu-Bonin region has evolved as a complex system of intraoceanic arcs, basins, and trenches since westward subduction of the Pacific Plate started in the early-middle Eocene (Ben-Avraham and Uyeda, 1983; Karig, 1975; Ogawa and Naka, 1984). The Pacific Plate is presently being subducted to the northwest at a rate of 8-10 $\mathrm{cm} \mathrm{yr}^{-1}$ beneath the active Izu-Bonin arc. The plate boundary is

\footnotetext{
' Fryer, P., Pearce, J. A., Stokking, L. B., et al., 1992. Proc. ODP. Sci. Results, 125: College Station, TX (Ocean Drilling Program).

${ }_{2}$ Institute of Oceanographic Sciences, Wormley, Surrey, GU8 5 UB, United Kingdom.

${ }^{3}$ Department of Geological Sciences, University of Durham, DH1 3LE, United Kingdom.

${ }^{4}$ Department of Geology, University of New England, Armidale, N.S.W. 23581. Australia.

${ }^{5}$ Hawaii Institute of Geophysics, University of Hawaii at Manoa, Honolulu, HI 96822 ,
} U.S.A. marked by the north-south striking Izu-Bonin Trench which is separated from the active volcanic arc by a $150-200 \mathrm{~km}$ wide forearc basin. The forearc basin is filled with volcaniclastic and hemipelagic sediments that lie to the arcward side of an outerarc high (Honza and Tamaki, 1985).

During ODP Leg 125, this outerarc high was drilled at Site 786, situated in the centre of the Izu-Bonin forearc $\left(31^{\circ} 52^{\prime} \mathrm{N}, 141^{\circ} 13.6^{\prime}\right.$ E) about $192 \mathrm{~km}$ east of Myojin Sho island in the active arc (Fig. 1). Four lithologic units were recovered from the two Holes (786A and 786B) drilled at this site. Units I, II, and III are recent to early-middle Eocene sediments found exclusively in Hole 786A. Unit IV represents igneous basement found at the base of Hole 786A and throughout Hole 786B. The drilling of Hole 786B was a major achievement of Leg 125 because it penetrated over $700 \mathrm{~m}$ into the Izu-Bonin forearc basement. Detailed examination of Unit IV has allowed a division into 34 subunits on the basis of lithological contrasts (dikes and sills, breccias, flows, sedimentary and tectonized horizons) and geochemistry (see van der Laan et al., this volume; Arculus et al., this volume). Arculus et al. identified eight major compositional groups within the igneous rocks based on petrological and geochemical characteristics. The resulting lithostratigraphy for the igneous basement at Site 786 is summarized in Figure 2.

This stratigraphy consists of a basal sequence of low-Ca boninite intrusive sheets overlain by low-Ca boninite pillow lavas. These in turn are overlain by intermediate-Ca bronzite-andesites, low-Ca bronzite-andesites, andesites, dacites, and rhyolites, which occur as a sequence of volcaniclastic breccias, lava flows, pyroclastic flows (including welded tuffs), and intrusive sheets. Clastic sediments, which were deposited in a shallow marine to subaerial environment (McCoy and LaGabrielle, this volume), are interbedded with the volcanic extrusives and breccias. Potassium/argon dating gives an isochron age of $41.3 \pm 0.5 \mathrm{Ma}$ age for this sequence (Mitchell et al., this volume), which is consistent with the middle Eocene nannofossil assemblages found both in the immediately overlying sediments and in those sediments intercalated within the lavas (Xu and Wise, this volume; Milner, this volume). The ca. $41 \mathrm{Ma}$ old intrusive sheets and overlying pillow lavas that form the base of the hole have been interpreted as an oceanic forearc basement of low-Ca boninite and low $\mathrm{Ca}$ bronzite-andesite composition ( $\mathrm{Ar}$ culus et al., this volume). Built upon this is a low- and intermediate-Ca boninite to rhyolite volcanic edifice of similar age. The Eocene forearc basement and edifice is cut by a suite of intermediate- $\mathrm{Ca}$ and high- $\mathrm{Ca}$ boninite intrusive sheets which give a K-Ar isochron age of $34.8 \pm 1.0 \mathrm{Ma}$ (Mitchell et al., this volume). The low sedimentation rate and low volcaniclastic input recorded in the Oligocene sediments of $35 \mathrm{Ma}$ age overlying the basement at Site 786 suggest that these late Oligocene dikes probably were 
A

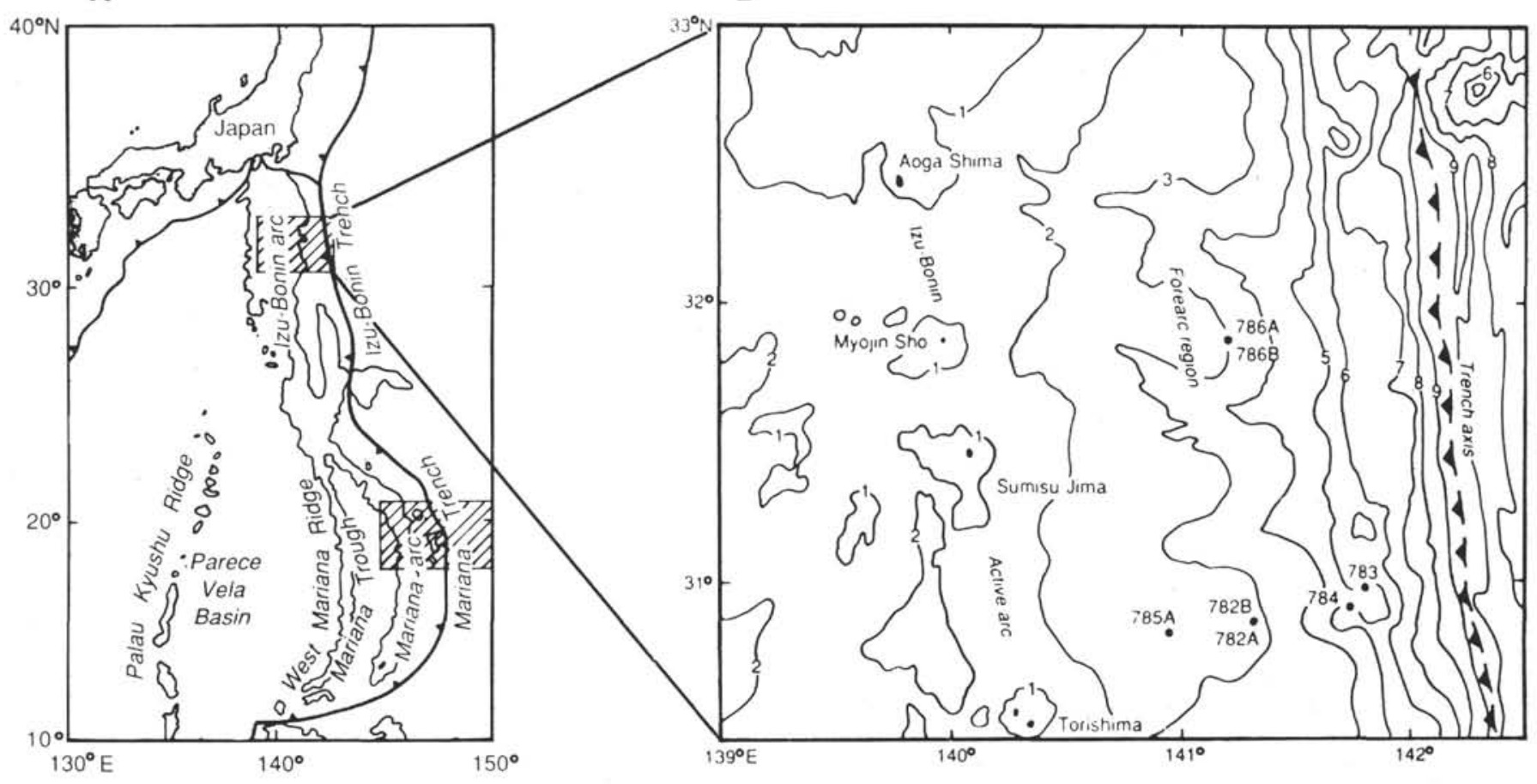

Figure 1. Location of ODP Leg 125 Site 786. A. Two areas studied by the JOIDES Resolution in the Mariana-Izu-Bonin (Ogasawara) region. B. Detailed setting of Site 786 within the northern Izu-Bonin forearc terrane. Bathymetric contours are in kilometers below sea level.

not locally derived but emplaced laterally over a considerable distance from outside the forearc region.

The igneous rocks encountered at Site 786 cover a remarkably diverse spectrum of compositions, ranging from boninites and bronzite andesites to andesites, dacites and rhyolites (see Fig. 3). The eight major chemical groups recognized by Arculus et al. (this volume) were distinguished primarily by the abundance levels of the majorelement oxides, $\mathrm{SiO}_{2}, \mathrm{MgO}$, and $\mathrm{CaO}$. In order of increasing $\mathrm{SiO}_{2}$ and decreasing $\mathrm{MgO}$ contents, the eight lithologic groups are as follows: (1) low-Ca boninite, (2) intermediate-Ca boninite, (3) high-Ca boninite, (4) low-Ca bronzite-andesite, (5) intermediate-Ca bronziteandesite, (6) andesite, (7) dacite, and (8) rhyolite. The main lithological, petrological, and geochemical characteristics of each chemical group are summarized in Table 1.

In this contribution we examine the trace-element characteristics of these chemical groups in order, (1) to verify the robustness of this classification scheme, and (2) to investigate possible relationships between the groups and the underlying petrogenetic causes. We then use the trace-element data to place constraints on the nature and composition of the sub-forearc mantle. Finally we discuss the results in the context of models for the evolution of the Izu-Bonin forearc basement.

\section{ANALYTICAL TECHNIQUES}

Approximately 150 samples were selected from representative lithologies in the Site 786 cores. Analyses were performed using $\mathrm{X}$-ray fluorescence (XRF) for major-elements and selected traceelements either onboard the JOIDES Resolution or at the University of New England, Australia. Samples prepared onboard ship were milled in a tungsten carbide barrel whereas those prepared in New England were ground in agate barrels. Full details of the XRF technique employed in both laboratories and an inter- laboratory comparison of analytical results are given in Arculus et al. (this volume). The XRF major and trace-element data are presented in Table 2.

A subset of 52 samples were analyzed by inductively coupled plasma mass spectrometry (ICP-MS) at the University of Durham, U.K. This technique allows data on a wide range of up to 34 traceelements to be obtained, including all rare-earth-elements, and $\mathrm{Ta}, \mathrm{Hf}$, $\mathrm{Pb}, \mathrm{Th}$, and $\mathrm{U}$. The overlap in several elements ( $\mathrm{Rb}, \mathrm{Sr}, \mathrm{Y}, \mathrm{Zr}, \mathrm{Nb}$, and many transition-elements) determined by both XRF and ICP-MS provide a check on data quality, and Figure 4 shows a comparison of $\mathrm{XRF}$ and ICP-MS data for three elements $\mathrm{Zr}$, Sr, and Y. The greater sensitivity of the ICP-MS technique is critical for these samples with inherently low incompatible trace-element abundances. $0.1 \pm 0.001 \mathrm{~g}$ of sample was weighed and digested by an $\mathrm{HF} / \mathrm{HNO}_{3}$ mixture and then by $\mathrm{HClO}_{4}$ in PTFE-ware. Samples were spiked by $\mathrm{Rh}, \mathrm{Re}$, and $\mathrm{Bi}$ internal standards, and run in a dilute nitric acid matrix at a $0.1 \mathrm{~g}$ $50 \mathrm{ml}^{-1}$ concentration of dissolved solids (i.e., total dilution factor of 500 ). Samples were run on a VG PQ1 instrument. A multi-element skipscan analytical program was chosen that had a dwell time of 320 $\mathrm{ms}$, and a total of $250 \mathrm{scans}$, for each mass number determined. The correction procedure included reagent blank and wash subtraction, drift monitoring, and correction for oxide/hydroxide interferences and isotopic overlaps. An initial calibration was performed using synthetic standards and finalized with a set of laboratory and international standards. Detection limits for all elements are 0.01-0.02 ppm (at $3 \mathrm{~s}$ of background) except for $\mathrm{Zr}, \mathrm{Nd}, \mathrm{Sm}, \mathrm{Gd}(0.05 \mathrm{ppm})$ and $\mathrm{Ba}$, $\mathrm{La}$, and $\mathrm{Pb}(0.1-0.2 \mathrm{ppm})$. Precision typically ranges from $15 \%$ at less than $10 \times$ detection limit, to about $3 \%$ at $100 \times$ detection limit. The ICP-MS trace element data are given in Table 3.

A small group of 21 samples was also analyzed for eight rare-earth elements ( $\mathrm{La}, \mathrm{Ce}, \mathrm{Nd}, \mathrm{Sm}, \mathrm{Eu}, \mathrm{Tb}, \mathrm{Yb}, \mathrm{Lu})$ and several other traceelements (Th, U, Ta, Hf, Sc, Co, Cs) by instrumental neutron activa- 
Table 1. Summary of lithological occurrences, mineralogical, features, ages, and selected chemical characteristics of the different groups distinguished at Site 786.

\begin{tabular}{|c|c|c|c|c|c|}
\hline Name, age, lithogical occurrence & Summary of phenocryst assemblage & & $\begin{array}{l}\text { or element } \\
\text { acteristics }\end{array}$ & $\mathrm{Ni}$ ar & $\mathrm{d}$ Cr abundances \\
\hline $\begin{array}{l}\text { Low-Ca boninite (LCB) } \\
\qquad 41 \mathrm{Ma} \\
\text { pillow lavas, breccias }\end{array}$ & $\begin{array}{l}5 \% \text { euhedral, fresh to altered, ol } \\
2 \%-5 \% \text { euhedral, fresh to altered, opx } \\
\text { trace clinopyroxene, Cr-spinel }\end{array}$ & $\begin{array}{l}\mathrm{SiO}_{2} \\
\mathrm{MgO} \\
\mathrm{CaO} \\
\mathrm{Al}_{2} \mathrm{O}_{3}\end{array}$ & $\begin{array}{c}55 \%-60 \% \\
10.7 \%-14.1 \% \\
3.1 \%-5.5 \% \\
11.4 \%-14.5 \%\end{array}$ & $\begin{array}{l}\mathrm{Ni} \\
\mathrm{Cr}\end{array}$ & $\begin{array}{c}190-310 \mathrm{ppm} \\
810-1200 \mathrm{ppm}\end{array}$ \\
\hline $\begin{array}{l}\text { Low-Ca bronzite - andesite (LCBA) } \\
\qquad \sim 41 \mathrm{Ma} \\
\text { dikes, pillow lavas }\end{array}$ & $\begin{array}{l}0 \%-5 \% \text { altered olivine } \\
0 \%-20 \% \text { altered orthopyroxene } \\
\text { trace clinopyroxene, } \mathrm{Cr} \text {-spinel } \\
\text { trace resorbed plagioclase }\end{array}$ & $\begin{array}{l}\mathrm{SiO}_{2} \\
\mathrm{MgO} \\
\mathrm{CaO} \\
\mathrm{Al}_{2} \mathrm{O}_{3}\end{array}$ & $\begin{array}{l}61 \%-65 \% \\
8.1 \%-12.1 \% \\
3.3 \%-6.4 \% \\
10.7 \%-12.6 \%\end{array}$ & $\begin{array}{l}\mathrm{Ni} \\
\mathrm{Cr}\end{array}$ & $\begin{array}{c}160-270 \mathrm{ppm} \\
670-1020 \mathrm{ppm}\end{array}$ \\
\hline $\begin{array}{l}\text { Intermediate-Ca bronzite (ICB) } \\
\qquad \begin{array}{c}-35 \mathrm{Ma} \text { dikes } \\
-41 \mathrm{Ma} \text { flows, breccias }\end{array}\end{array}$ & $\begin{array}{c}1 \%-5 \% \text { euhedral altered olivine } \\
5 \%-10 \% \text { euhedral orthopyroxene } \\
2 \%-4 \% \text { euhedral-corroded cpx } \\
0 \%-10 \% \text { euhedral-corroded plag } \\
\text { trace } C r \text {-spinel }\end{array}$ & $\begin{array}{l}\mathrm{SiO}_{2} \\
\mathrm{MgO} \\
\mathrm{CaO} \\
\mathrm{Al}_{2} \mathrm{O}_{3}\end{array}$ & $\begin{array}{c}53 \%-58 \% \\
8.5 \%-13.7 \% \\
6.9 \%-8.5 \% \\
12.4 \%-14.4 \%\end{array}$ & $\begin{array}{l}\mathrm{Ni} \\
\mathrm{Cr}\end{array}$ & $\begin{array}{l}150-370 \mathrm{ppm} \\
470-920 \mathrm{ppm}\end{array}$ \\
\hline $\begin{array}{c}\text { Intermediate-Ca bronzite-andesite (ICBA) } \\
\qquad \begin{array}{c}\sim 1 \mathrm{Ma} \\
\text { dikes, lavas, breccias }\end{array}\end{array}$ & $\begin{array}{c}0 \%-1 \% \text { altered olivine } \\
6 \%-20 \% \text { euhedral orthopyroxene } \\
3 \%-10 \% \text { euhedral clinopyroxene } \\
5 \%-15 \% \text { euhedral-resorbed plag } \\
\text { trace } \mathrm{Cr} \text {-spinel }\end{array}$ & $\begin{array}{l}\mathrm{SiO}_{2} \\
\mathrm{MgO} \\
\mathrm{CaO} \\
\mathrm{Al}_{2} \mathrm{O}_{3}\end{array}$ & $\begin{array}{c}56 \%-63 \% \\
4.8 \%-9.1 \% \\
4.6 \%-8.7 \% \\
11.9 \%-16.2 \%\end{array}$ & $\begin{array}{l}\mathrm{Ni} \\
\mathrm{Cr}\end{array}$ & $\begin{array}{c}60-220 \mathrm{ppm} \\
170-950 \mathrm{ppm}\end{array}$ \\
\hline $\begin{array}{l}\text { High-Ca boninite }(\mathrm{HCB}) \\
\sim 35 \mathrm{Ma} \\
\text { dikes, sills }\end{array}$ & $\begin{array}{l}\text { Euhedral altered olvine } \\
\text { trace euhedral orthopyroxene } \\
1 \%-3 \% \text { euhedral-corroded cpx } \\
\text { trace plag, dark-red Cr-spinel } \\
\text { glomerocrysts of opx }+ \text { cpx }+ \text { tr. plag }\end{array}$ & $\begin{array}{l}\mathrm{SiO}_{2} \\
\mathrm{MgO} \\
\mathrm{CaO} \\
\mathrm{Al}_{2} \mathrm{O}_{3}\end{array}$ & $\begin{array}{c}50 \%-56 \% \\
6.2 \%-12.2 \% \\
9.4 \%-14.4 \% \\
12.0 \%-15.5 \%\end{array}$ & $\begin{array}{l}\mathrm{Ni} \\
\mathrm{Cr}\end{array}$ & $\begin{array}{c}170-380 \mathrm{ppm} \\
330-1010 \mathrm{ppm}\end{array}$ \\
\hline $\begin{array}{c}\text { Andesite (AND) } \\
\sim 41 \mathrm{Ma} \\
\text { dikes, breccias }\end{array}$ & $\begin{array}{l}0 \% \text {-trace orthopyroxene } \\
1 \%-3 \% \text { clinopyroxene } \\
3 \%-5 \% \text { euhedral plagioclase } \\
\text { some phenocryst magnetite }\end{array}$ & $\begin{array}{l}\mathrm{SiO}_{2} \\
\mathrm{MgO} \\
\mathrm{CaO} \\
\mathrm{Al}_{2} \mathrm{O}_{3}\end{array}$ & $\begin{array}{c}56 \%-63 \% \\
1.8 \%-5.7 \% \\
5.4 \%-8.2 \% \\
15.1 \%-18.5 \%\end{array}$ & $\begin{array}{l}\mathrm{Ni} \\
\mathrm{Cr}\end{array}$ & $\begin{array}{l}10-50 \mathrm{ppm} \\
0-100 \mathrm{ppm}\end{array}$ \\
\hline $\begin{array}{c}\text { Dacite (DAC) } \\
-41 \mathrm{Ma} \\
\text { dikes, flows, breccias }\end{array}$ & $\begin{array}{c}0 \%-5 \% \text { euhedral-corroded opx } \\
\text { trace-2\% euhedral-resorbed cpx } \\
3 \%-10 \% \text { plagioclase } \\
\text { phenocryst magnetite }\end{array}$ & $\begin{array}{l}\mathrm{SiO}_{2} \\
\mathrm{MgO} \\
\mathrm{CaO} \\
\mathrm{Al}_{2} \mathrm{O}_{3}\end{array}$ & $\begin{array}{c}65 \%-67 \% \\
0.6 \%-3.4 \% \\
4.0 \%-5.8 \% \\
14.9 \%-15.8 \%\end{array}$ & $\begin{array}{l}\mathrm{Ni} \\
\mathrm{Cr}\end{array}$ & $\begin{array}{l}0-30 \mathrm{ppm} \\
0-50 \mathrm{ppm}\end{array}$ \\
\hline $\begin{array}{c}\text { Rhyolite (RHY) } \\
\sim 41 \mathrm{Ma} \\
\text { dikes, flows }\end{array}$ & $\begin{array}{c}0 \% \text {-trace euhedral orthopyroxene } \\
0 \% \text {-trace euhedral-anhedral cpx } \\
\text { trace- } 3 \% \text { resorbed plagioclase } \\
\text { trace quartz } \\
\text { phenocryst magnetite common }\end{array}$ & $\begin{array}{l}\mathrm{SiO}_{2} \\
\mathrm{MgO} \\
\mathrm{CaO} \\
\mathrm{Al}_{2} \mathrm{O}_{3}\end{array}$ & $\begin{array}{c}71 \%-77 \% \\
0.0 \%-1.5 \% \\
0.5 \%-3.5 \% \\
12.2 \%-14.2 \%\end{array}$ & $\begin{array}{l}\mathrm{Ni} \\
\mathrm{Cr}\end{array}$ & $\begin{array}{l}0-15 \mathrm{ppm} \\
0-30 \mathrm{ppm}\end{array}$ \\
\hline
\end{tabular}

tion analysis (INAA) at the Open University, U.K. Analytical details can be found in Potts et al. (1985). The INAA data are presented in Table 4. Three samples analysed by both INAA and ICP-MS have been plotted for comparison on a chondrite-normalized rare-earth element diagram (Fig. 5) in order to illustrate the good agreement between the two techniques.

\section{CHARACTERISTICS OF THE CHEMICAL GROUPS}

The XRF trace-element data are presented as $\mathrm{SiO}_{2}$ variation diagrams in Figure 6. It can be seen from these plots that the chemical groups proposed on major-element and petrological grounds can also be resolved as distinct groups on the abundances of many trace-elements, notably $\mathrm{Ni}$ and $\mathrm{Cr}$ (see also Table 1) and $\mathrm{Zr}$ and $\mathrm{Sr}$. However, the range in $\mathrm{P}_{2} \mathrm{O}_{5}$ and $\mathrm{Y}$ contents of most groups overlap and are quite variable, with several samples exhibiting a marked enrichment in both elements which exceeds that attributable to primary magmatic variations. Although $\mathrm{P}$ and $\mathrm{Y}$ are usually considered to behave as immobile elements during alteration processes, it seems that they can be mobilized during the alteration of boninite glass (cf. Taylor et al., in press). This is discussed in more detail in the alteration section below. The following sections summarize the stratigraphic, major and trace-element, and petrological characteristics of the eight chemical groups.

\section{Boninites and Bronzite-Andesites}

\section{Low-Ca Boninites}

Low-Ca boninites (LCB) of ca. $41 \mathrm{Ma}$ age occur as dikes at the base of Hole 786B, and as pillow lavas in Cores 57R to 63R, where they are interbedded with low-Ca bronzite-andesites. They form a distinct group together with the low-Ca bronzite andesites on most element variation diagrams (Fig. 6). They have similar Ni contents (150-300 ppm) to the other boninite groups but are displaced to higher $\mathrm{SiO}_{2}$ (55.4-57.7 wt\%). Cr contents for the LCB's range from 625 to $1200 \mathrm{ppm}$ (average $986 \mathrm{ppm}$ ), which is higher than both other boninite types. They have the lowest $\mathrm{TiO}_{2}$ of all the boninite groups (average of $0.21 \mathrm{wt} \%$ ). $\mathrm{Rb}$ (and $\mathrm{K}_{2} \mathrm{O}$ ) are quite variable in the $\mathrm{LCB}$

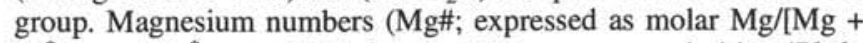
$\mathrm{Fe}^{*}$ ]; note: $\mathrm{Fe}^{*}$ as total $\mathrm{Fe}$ ) for the $\mathrm{LCB}$ group are primitive (72.674.8 ), and are in equilibrium with mantle olivine of composition $\mathrm{Fo}_{89-90}$ (Roeder and Emslie, 1970). They contain 2-5 modal percent of phenocrysts of olivine (composition $\mathrm{FO}_{89-90}$, with chromium-spinel inclusions), and enstatite and/or clinoenstatite.

\section{Low-Ca-Bronzite-Andesites}

Low-Ca bronzite-andesites (LCBA) of $41 \mathrm{Ma}$ age occur with LCB's as dikes at the base of Hole 786B (Cores 68R-72R) and 


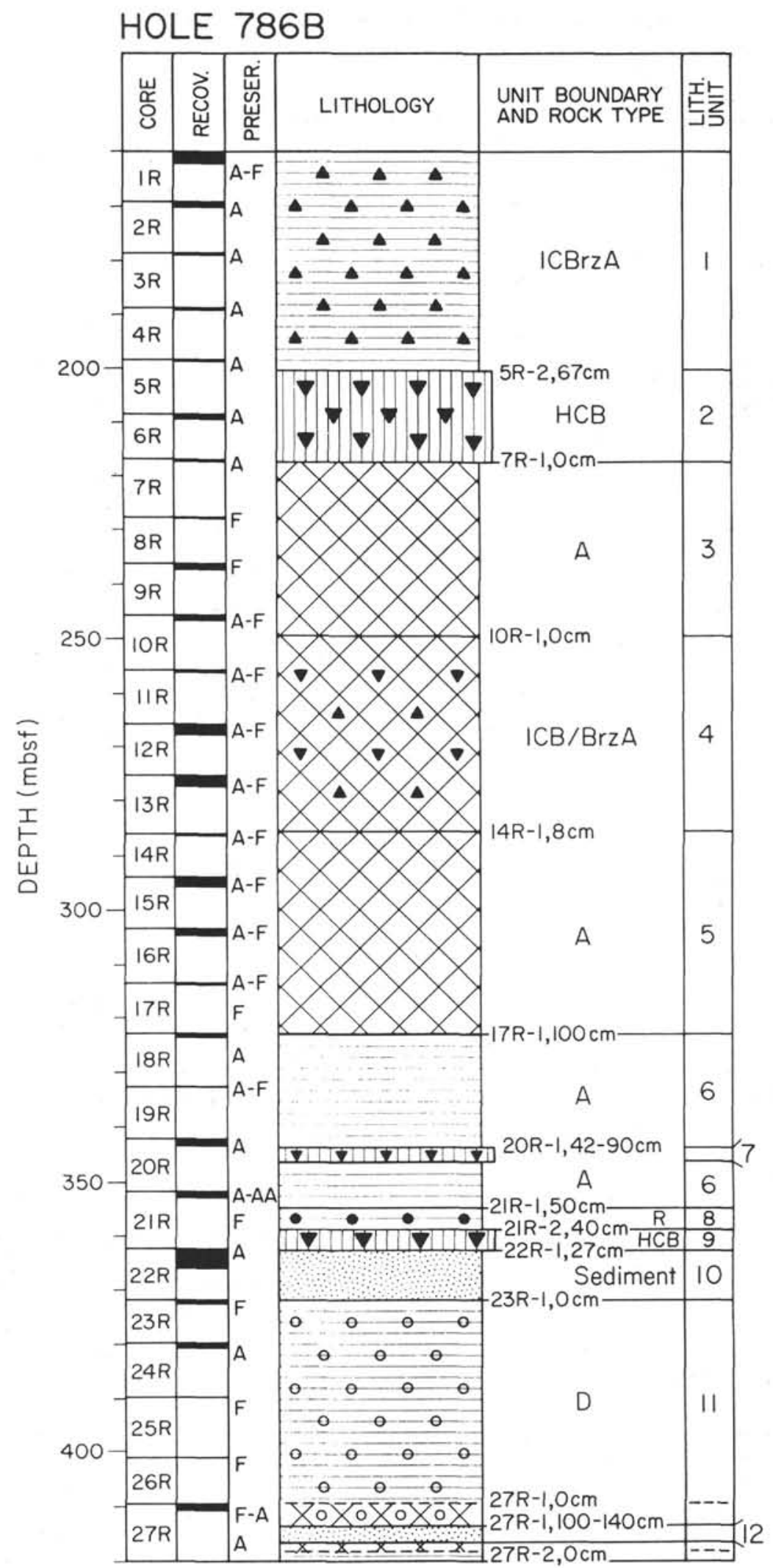

Figure 2. Simplified summary of lithostratigraphy recovered at Site 786. 
HOLE 786B (continued)

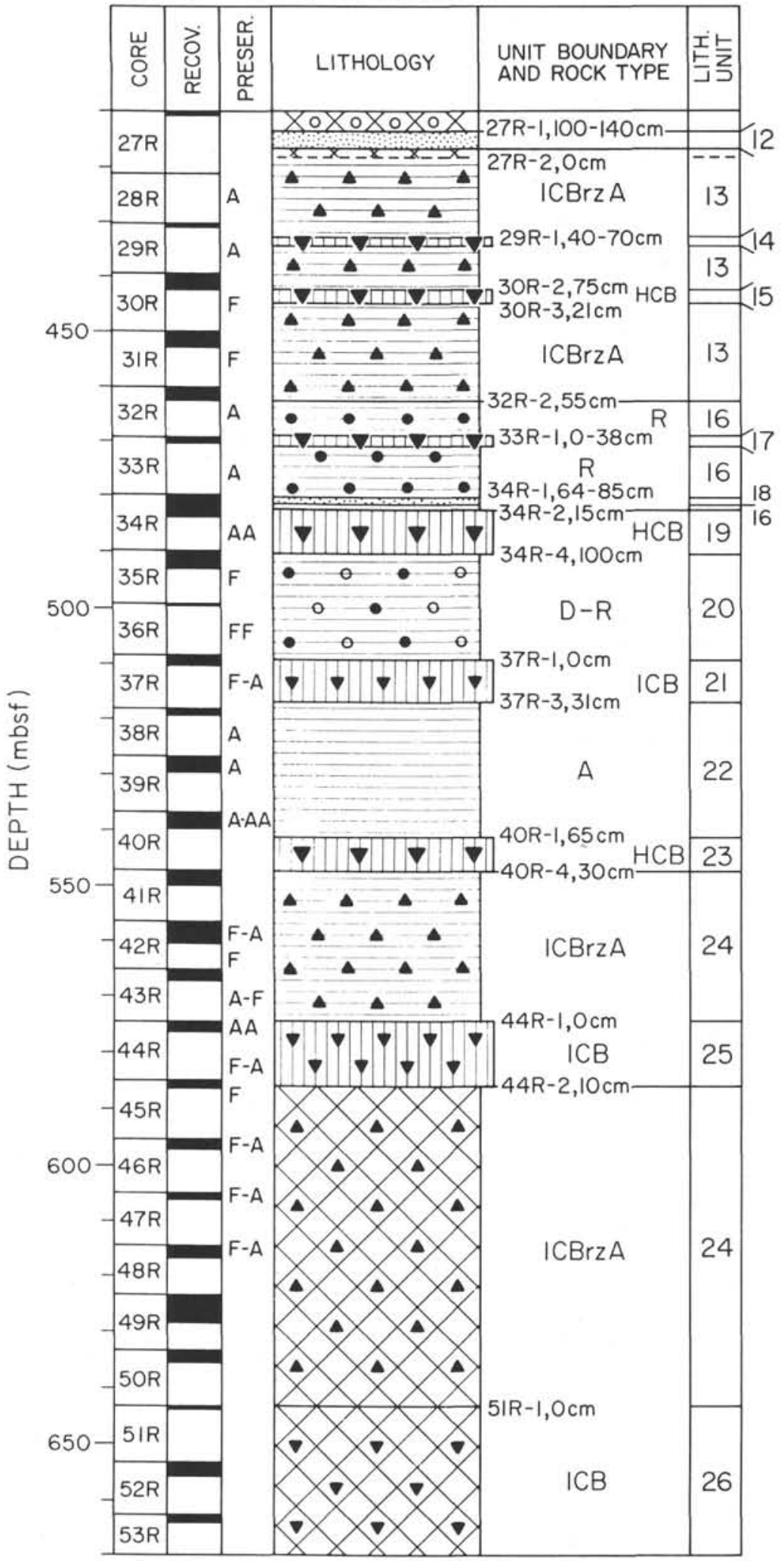

Figure 2 (continued). 


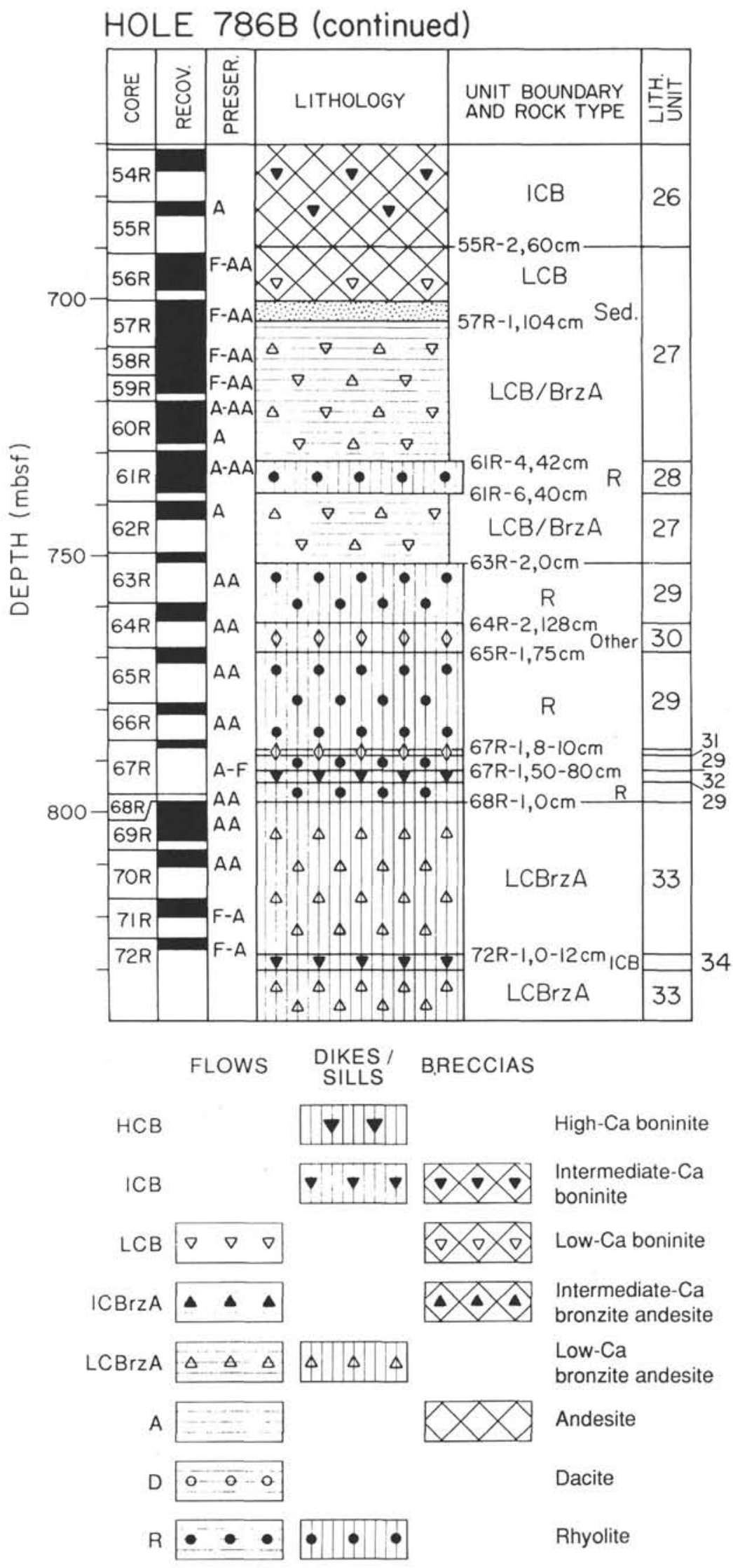

Figure 2 (continued). 


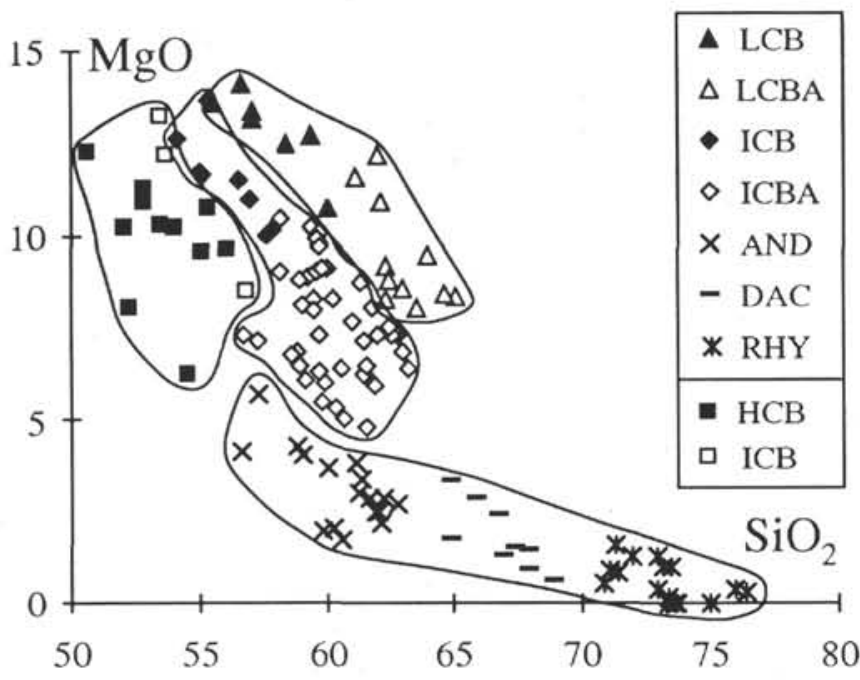

Figure $3 . \mathrm{MgO}$ vs. $\mathrm{SiO}_{2}$ co-variation diagram for the eight main lithological groups recovered from Site 786. $\mathrm{LCB}=$ low-Ca boninite, $\mathrm{LCBA}=$ low-Ca bronzite-andesite, $\mathrm{ICB}=$ intermediate-Ca boninite, ICBA $=$ intermediate-Ca bronzite-andesite, $\mathrm{AND}=$ andesite, $\mathrm{DAC}=$ dacite, $\mathrm{RHY}=$ rhyolite, $\mathrm{HCB}=$ high-Ca boninite. Square symbols represent late-stage dikes.

interbedded pillow lavas in cores 57R-63R. Compared to the LCB's, they have lower $\mathrm{MgO}$ (8.1-12.2 wt\%), $\mathrm{Mg} \#(67.5-74.0 \mathrm{wt} \%)$, and $\mathrm{CaO}$ (3.3-6.4 wt\%), and higher $\mathrm{SiO}_{2}(62.4-64.1 \mathrm{wt} \%)$. They are less primitive than any of the boninites, with lower Ni contents (103-269 ppm), but have similar $\mathrm{Cr}$ contents (676-1017 ppm; average 805 ). Although they have similar $\mathrm{TiO}_{2}(0.18-0.24$; average $0.22 \mathrm{wt} \%)$ to the LCB's, V is lower in the LCBA's. Abundances of $\mathrm{Zr}$ (31-61 ppm), $\mathrm{Y}(5-10 \mathrm{ppm})$, and $\mathrm{P}_{2} \mathrm{O}_{5}(0.6-1.3 \mathrm{wt} \%)$ in the LCBA's increase with increasing $\mathrm{wt} \% \mathrm{SiO}_{2}$, and are also greater than those in the LCB group. $\mathrm{Rb}$ contents are the lowest of all the groups $(<6 \mathrm{ppm})$, but are variable like $\mathrm{Ba}$, probably as a result of alteration. The LCB's and LCBA's show a trend of gradually increasing $\mathrm{Sr}$ as $\mathrm{SiO}_{2}$ increases that is displaced to lower $\mathrm{Sr}$ contents $(100-150 \mathrm{ppm})$ relative to the other boninite and bronzite-andesite groups. The LCBA's are crystal rich with 20-35 modal percent phenocrysts of: olivine ( $0 \%-1 \%$ : composition $\mathrm{Fo}_{86.6-87.1}$, with chromium-spinel inclusions and orthopyroxene jackets), orthopyroxene $(2 \%-20 \%)$, augite $(0 \%-10 \%)$, and plagioclase $(0 \%-3 \%)$.

\section{Intermediate-Ca Boninites}

Some intermediate-Ca boninites (ICB) occur as Oligocene-age dikes of $35 \mathrm{Ma}$, and breccias of indeterminate origin and/or age interbedded with intermediate-Ca-bronzite-andesites, and are treated together with the HCB dikes. The rest of the ICB group is part of the Eocene edifice and is considered here. Compared with the LCB's, the ICB's form a distinct group with a lower range in $\mathrm{MgO}$ (from 6.8 to $13.3 \mathrm{wt} \%$ ), low $\mathrm{SiO}_{2}(53.7-59.3 \mathrm{wt} \%$ ), low $\mathrm{Mg}$ \# (from 60.7 to 71.5 ), and high $\mathrm{CaO}$ (from 3.06 to $8.2 \mathrm{wt} \%$ ). They are slightly less primitive than the LCB's, with lower Ni contents (150-220 ppm), lower $\mathrm{Cr}$ contents (393-921 ppm; average 653), and correspondingly higher $\mathrm{TiO}_{2}$ (average of $0.25 \mathrm{wt} \%$ ). They also have higher $\mathrm{Zr}$ and $\mathrm{Sr}$ contents than the other boninite groups ( $\mathrm{Zr} 30-40 \mathrm{ppm}, \mathrm{Sr} 140-190 \mathrm{ppm}$ ). They commonly contain 5-17 modal percent phenocrysts of enstatite, augite and olivine (composition $\mathrm{Fo}_{86-87.1}$, with chromium-spinel inclusions and enstatite jackets).

\section{Intermediate-Ca Bronzite-Andesites}

Intermediate-Ca bronzite-andesites (ICBA) of ca. 41 Ma age occur as flows (Cores 1R-5R and 27R-32R) and as interbedded breccia with ICB and dacite (Cores $10 \mathrm{R}-14 \mathrm{R}$ and $45 \mathrm{R}-51 \mathrm{R}$ ). Compared to the LCBA's, they have lower MgO (4.8-9.6 wt\%), $\mathrm{SiO}_{2}$ (59.0-62.8 wt\%) and $\mathrm{Mg \#} \mathrm{(52.9-62.0),} \mathrm{and} \mathrm{higher} \mathrm{CaO}(4.5-10.3$ wt\%). They are less primitive than the LCBA's with low Ni contents (61-144 ppm), low Cr contents (165-564 ppm, average 398) and high $\mathrm{TiO}_{2}(0.12-0.41 \mathrm{wt} \%)$. Like the ICB's, the ICBA's have elevated $\mathrm{Zr}$ and $\mathrm{Sr}$ contents (28-48 ppm and 130-190 ppm respectively) but are displaced to higher $\mathrm{SiO}_{2}$. Crystal contents vary from 0 to 13 modal percent of: orthopyroxene $(0 \%-3 \%)$, augite $(0 \%-3 \%)$, and plagioclase $(1 \%-10 \%)$. Magnetite and rare amphibole are present in the groundmass.

\section{Late High-Ca and Intermediate-Ca Boninite Dikes}

The late-stage intermediate-Ca and high-Ca boninites (ICB's and HCB's) of ca. $35 \mathrm{Ma}$ age occur exclusively as dikes throughout the basement sequence. Compared with the Eocene intermediate-Ca boninites, they form a distinct group with a lower range in $\mathrm{MgO}(6.3-$ $12.2 \mathrm{wt} \%)$, relatively low $\mathrm{SiO}_{2}(50.8-56.4 \mathrm{wt} \%)$, and low $\mathrm{Mg \#}$ (55.9-70.8). They are best distinguished from the other two boninite groups by their higher $\mathrm{CaO}(9.4-14.4 \mathrm{wt} \%)$, and correspondingly high $\mathrm{TiO}_{2}$ (average of $0.3 \mathrm{wt} \%$ ). They are as primitive as the Eocene ICB's, having only slightly lower Ni contents (179-321 ppm) but
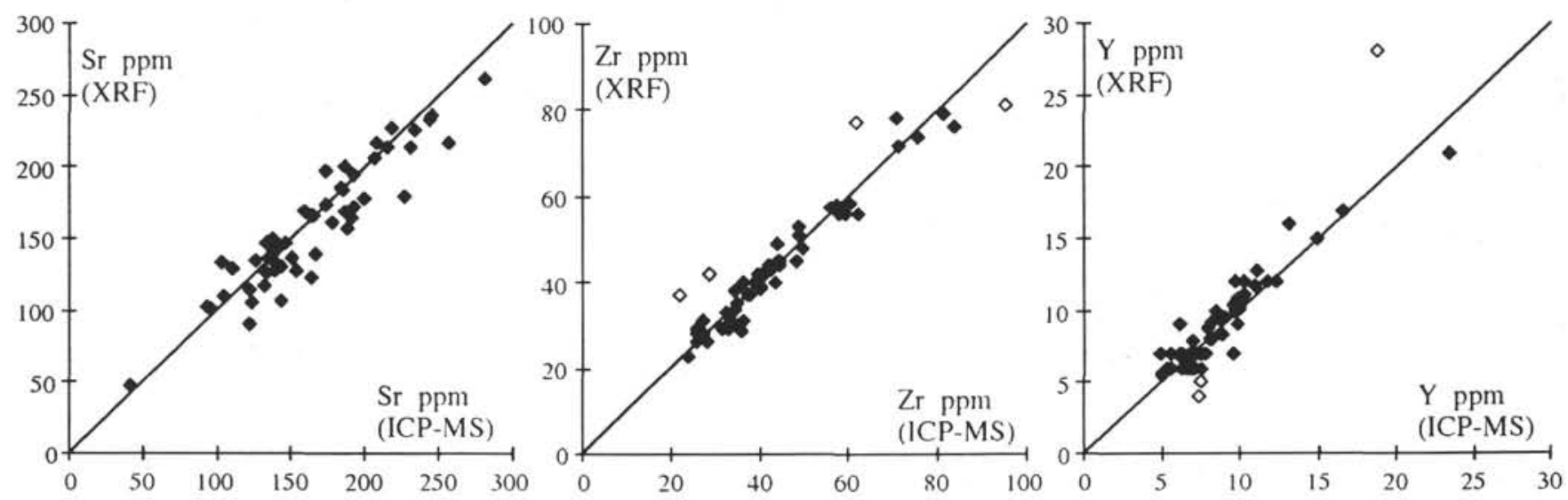

Figure 4. Comparison of XRF and ICP-MS techniques for three elements ( $\mathrm{Sr}, \mathrm{Zr}, \mathrm{Y})$ for samples from Site 786. 


\begin{tabular}{|c|c|c|c|c|c|c|c|c|c|c|c|c|c|c|c|c|c|c|c|c|c|c|c|c|c|c|c|c|c|}
\hline Hole & Core & $\begin{array}{c}\text { Interval } \\
\text { (cm) }\end{array}$ & $\begin{array}{l}\text { Depth } \\
\text { (mblsf) }\end{array}$ & Lab & $\begin{array}{l}\text { Lith, } \\
\text { unit }\end{array}$ & $\begin{array}{c}\text { Magma } \\
\text { series }\end{array}$ & $\begin{array}{l}\text { Chemical } \\
\text { group }\end{array}$ & $\mathrm{SiO}_{2}$ & $\mathrm{TiO}_{2}$ & $\mathrm{Al}_{3} \mathrm{O}_{3}$ & $\mathrm{Fe}_{2} \mathrm{O}_{3}$ & Mno & $\mathrm{MgO}$ & CaO & $\mathrm{Na}_{2} \mathrm{O}$ & $\mathrm{K}_{2} \mathrm{O}$ & $\mathrm{P}_{2} \mathrm{O}_{5}$ & $\mathbf{s}$ & LOI & $\mathrm{Ni}$ & Cr & $v$ & $\mathrm{Zn}$ & $c_{u}$ & Sc & $\mathrm{Ga}$ & $\mathrm{Rb}$ & Sr & $\mathrm{Y}$ \\
\hline & -2 & & 0.80 & odp & 2 & kes & НСB & 52,32 & 0.37 & 15.00 & 8.90 & 0.11 & $?$ & 11.34 & 2. & & & & & 170 & 3 & 237 & 63 & 63 & & & 6 & & \\
\hline $\mathrm{B}$ & $\begin{array}{l}6 \mathrm{R}-2 \\
6 \mathrm{R}-3\end{array}$ & $\begin{array}{l}128-130 \\
17-22\end{array}$ & $\begin{array}{l}210.98 \\
211.39\end{array}$ & $\begin{array}{l}\text { odp } \\
\text { une }\end{array}$ & 2 & $\begin{array}{l}\text { edykes } \\
\text { edykes }\end{array}$ & $\begin{array}{l}\text { HCB } \\
\text { HCB }\end{array}$ & $\begin{array}{r}52.82 \\
5401\end{array}$ & $\begin{array}{l}0.36 \\
0.33\end{array}$ & $\begin{array}{l}14.50 \\
14.84\end{array}$ & $\begin{array}{l}8.79 \\
8.58\end{array}$ & $\begin{array}{l}0.17 \\
0.17\end{array}$ & 21 & $\begin{array}{l}9.57 \\
942\end{array}$ & $\begin{array}{l}2.27 \\
2.19\end{array}$ & 0.26 & 0.05 & & & & 501 & 132 & 68 & 69 & & & & & \\
\hline & $2 \mid \mathrm{R}-2$ & $72-76$ & & $\begin{array}{l}\text { une } \\
\text { dodp }\end{array}$ & $\frac{2}{9}$ & dy & HCB & & 0 & $\begin{array}{l}14.84 \\
12.73\end{array}$ & 8.878 & 17 & & & 2,19 & 0 & & & & & 64 & & 67 & 54 & 32 & "I & & & \\
\hline & & 102 & 355.46 & une & 9 & dykes & HCB & 55.40 & 0.22 & 12 & 8.43 & 16 & 10.74 & 9.37 & $\begin{array}{l}2.37 \\
2.56\end{array}$ & 0.26 & $\begin{array}{l}0.08 \\
0.04\end{array}$ & & $\begin{array}{l}5.75 \\
6.02\end{array}$ & $\begin{array}{l}188 \\
275\end{array}$ & $\begin{array}{l}889 \\
1004\end{array}$ & $\begin{array}{l}185 \\
165\end{array}$ & 50 & $\begin{array}{l}40 \\
63\end{array}$ & 31 & 10 & $\begin{array}{l}8 \\
5\end{array}$ & 33 & \\
\hline & & 63 & 430.73 & & 14 & & $\mathrm{HCB}$ & & 0.21 & 12.02 & 8.17 & 20 & 10.88 & 13 & 2.17 & 0.19 & 0.20 & & 7.08 & 245 & 956 & 160 & 76 & 34 & 36 & 8 & 5 & 138 & 5 \\
\hline & $30 \mathrm{~K}$ & $136-1$ & 442.63 & une & 15 & 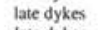 & НСB & & 0.40 & 14.95 & 8.65 & 0.017 & & 9.36 & 2.39 & 0.26 & 0.06 & & & 4 & 552 & 231 & 65 & 57 & 35 & 13 & 4 & 130 & 10 \\
\hline & $34 R-3$ & 45.47 & 481.25 & odp & 19 & edykes & HCB & 55.12 & 0.22 & 12.28 & 7.88 & 0.13 & 9.52 & 11.68 & 2.52 & 0 & 0.03 & 0 & $\begin{array}{l}5.44 \\
\end{array}$ & 277 & 769 & 160 & 55 & 61 & & & 15 & & 7 \\
\hline & 4082 & $\begin{array}{r}14-20 \\
54-58\end{array}$ & 2.84 & une & 19 & dykes & $\begin{array}{l}\mathrm{HCB} \\
\mathrm{H}\end{array}$ & & 0.21 & $\begin{array}{l}12.15 \\
1293\end{array}$ & $\begin{array}{l}8.29 \\
8863\end{array}$ & & $\begin{array}{r}9.59 \\
0.73\end{array}$ & & 2.65 & & & & & 246 & & 145 & 48 & 47 & 35 & 7 & 15 & 128 & 6 \\
\hline & $\begin{array}{l}40 R-2 \\
40-2\end{array}$ & $\begin{array}{l}54 \\
83\end{array}$ & 5 & odp & ${ }_{23}^{23}$ & $\begin{array}{l}\text { dykes } \\
\text { dykkes }\end{array}$ & $\begin{array}{l}\mathrm{HCB} \\
\mathrm{HCB}\end{array}$ & & $\begin{array}{l}0.30 \\
0.29\end{array}$ & 12 & 8 & & & & 2 & $\begin{array}{l}0 \\
0 \\
0\end{array}$ & $\begin{array}{l}04 \\
02 \\
02\end{array}$ & & $\begin{array}{l}5.46 \\
8000\end{array}$ & 374 & 0 & 193 & $\begin{array}{l}57 \\
59\end{array}$ & $\begin{array}{l}27 \\
34\end{array}$ & 38 & 10 & ${ }_{7}^{6}$ & 36 & $\begin{array}{l}9 \\
8\end{array}$ \\
\hline & $\begin{array}{l}40 X-1 \\
16 X C C\end{array}$ & & & $\begin{array}{l}\text { une } \\
\text { odp }\end{array}$ & $\begin{array}{r}23 \\
1\end{array}$ & $\begin{array}{l}\text { e dykes } \\
\text { dyykes }\end{array}$ & 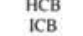 & & $\begin{array}{l}0 . \\
0 .\end{array}$ & $\begin{array}{l}13 \\
15\end{array}$ & & & & & 2 & 0 & . & & & 19 & & $\begin{array}{l}87 \\
26\end{array}$ & 68 & $\begin{array}{l}34 \\
37\end{array}$ & 38 & 10 & 3 & $\begin{array}{l}1.97 \\
147\end{array}$ & 9 \\
\hline & $x-1$ & $20-20$ & & & $i$ & dykes & ICB & & 0 & is. & o. & & & & & & & & & & & & & & & & & & \\
\hline & $20 \mathrm{R}-1$ & $43-46$ & 343.73 & & $\therefore$ & 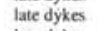 & ICB & & 0 & 12. & 8.49 & & & 8 & & & 0.01 & & & & & & & & & & & & \\
\hline & 206 & & & & , & s. & ICB & & 0. & 13 & & & & & & & & & & & & & & & & & & & \\
\hline & $37 R-3$ & 13 & 45 & une & 21 & 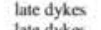 & ICB & & 0.26 & 14.59 & 8.20 & & & 8.2 & 2.8 & 0. & 0.04 & & & 193 & 24 & 196 & 69 & 21 & 31 & & & 153 & \\
\hline & $44 R-1$ & 38 & 98 & ne & 25 & 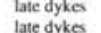 & ICB & & o, & & $\begin{array}{l}9.20 \\
0.40\end{array}$ & & & & & & & & & & & 190 & 62 & 44 & 28 & & & 117 & 8 \\
\hline & $44 R-1$ & $102-109$ & .62 & ne & 25 & & KCB & 3.48 & 0. & 13. & 9.11 & 0.22 & 8.17 & 8.0 & 2.30 & 0. & 0. & 0.00 & & 36 & 9 & 93 & 67 & 157 & 26 & 9 & 8 & 107 & \\
\hline $6 \mathrm{~B}$ & $36 \mathrm{R}-2$ & $51-56$ & 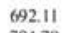 & une & 27 & 3 ser & LCB & 3 & 0.22 & 12.13 & 8.10 & 0.1 & 13.14 & 5.02 & 2.86 & 1.2 & 0.01 & 0.00 & 11.00 & 197 & 4 & 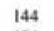 & 55 & 33 & & 11 & 15 & 19 & \\
\hline B & $57 R-4$ & 69 & 70 & 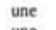 & ${ }^{27}$ & es & LCB & & 0.20 & & 8.11 & & & 5 & 3. & 0 & & & & 281 & 1200 & 134 & 61 & 18 & 29 & 9 & 9 & 106 & 4 \\
\hline & & $116-120$ & & 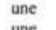 & ${ }^{27}$ & & LCB & & $\begin{array}{l}0 . \\
0 .\end{array}$ & & 8.21 & & & & & & & & & $\begin{array}{r}281 \\
289\end{array}$ & & & & & 26 & 12 & 1 & 91 & ? \\
\hline $786 \mathrm{~B}$ & $\begin{array}{l}99 \mathrm{R} \cdot 3-3 \\
2 \mathrm{R}-3\end{array}$ & $\begin{array}{l}84.9 \\
404\end{array}$ & & : & $\begin{array}{l}27 \\
27\end{array}$ & $\begin{array}{l}\text { ies } \\
\text { ies }\end{array}$ & $\begin{array}{l}\text { LCB } \\
\text { LCB }\end{array}$ & & . & $\begin{array}{l}11 \\
12\end{array}$ & $\begin{array}{l}8.46 \\
8.77\end{array}$ & & & 5 & 3. & 0. & & & & $\begin{array}{l}9 \\
6\end{array}$ & & $\begin{array}{l}144 \\
166\end{array}$ & $\begin{array}{l}61 \\
63\end{array}$ & $\begin{array}{l}29 \\
60\end{array}$ & 28 & 9 & & $\begin{array}{r}91 \\
91\end{array}$ & $\begin{array}{l}6 \\
8\end{array}$ \\
\hline 7868 & & $11-17$ & 811.32 & $\begin{array}{l}\text { opp } \\
\text { une }\end{array}$ & ${ }_{33}^{27}$ & $\begin{array}{l}\text { Bs sertes } \\
\text { B series }\end{array}$ & $\angle C B$ & & . & $\begin{array}{l}12.52 \\
14.47\end{array}$ & $\begin{array}{l}8.113 \\
8.13\end{array}$ & & & 3 & 2 & 0 & & & & $\begin{array}{l}6 \\
2 \\
\end{array}$ & 032 & $\begin{array}{l}106 \\
205\end{array}$ & $\begin{array}{l}63 \\
65\end{array}$ & $\begin{array}{l}60 \\
80\end{array}$ & 29 & 11 & & 104 & \\
\hline $786 \mathrm{~B}$ & & & & une & 27 & & LCB & & 0.32 & & $?$ & & & & & & & & & & & & 0. & 75 & & 9 & & & \\
\hline $86 \mathrm{H}$ & $\underset{58 R-2}{57 R-2}$ & $124-128$ & $\begin{array}{c}702.46 \\
7\end{array}$ & une & ${ }_{27}^{27}$ & Bseries & LCB & & 0.24 & 11.85 & 10.15 & & & 4.5 & 3.0 & 0.9 & 0. & & 12.4 & & 1014 & & 72 & 18 & & 11 & 12 & 107 & \\
\hline $786 \mathrm{~B}$ & $\begin{array}{l}58 R-3 \\
60 R-3\end{array}$ & $\begin{array}{r}62-69 \\
102-108\end{array}$ & $\begin{array}{l}721283 \\
722.77\end{array}$ & $\begin{array}{l}\text { une } \\
\text { une }\end{array}$ & $\begin{array}{l}27 \\
27\end{array}$ & $\begin{array}{l}\text { LCB Bsties } \\
\text { LCB serites }\end{array}$ & $\begin{array}{l}\text { LCBA } \\
\text { LCBA }\end{array}$ & & , & 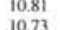 & $\begin{array}{l}6.99 \\
7.16\end{array}$ & & & 5.0 & 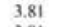 & 0.11 & & & 7.2 & & 1017 & & & & & 8 & & & \\
\hline $786 \mathrm{~B}$ & $62 R-1$ & $114-120$ & 739.54 & & ${ }_{27}^{27}$ & $\begin{array}{l}\text { ies } \\
\text { ies }\end{array}$ & LC & & 0 & & & & & & 3.8 & 0 & & & & & & 203 & 53 & 43 & 25 & 11 & & 80 & \\
\hline $786 \mathrm{~B}$ & $69 R-1$ & $65-67$ & 797.95 & & 33 & B series & LCBA & & 0 & & 6.9 & & $\begin{array}{l}1.214 \\
9.46\end{array}$ & 3. & 3. & 0 & $\begin{array}{l}0.02 \\
0.04\end{array}$ & & $\begin{array}{l}9.58 \\
4.31\end{array}$ & ${ }_{21}^{26}$ & 977 & $\begin{array}{l}121 \\
156\end{array}$ & $\begin{array}{l}58 \\
75\end{array}$ & $\begin{array}{l}145 \\
177\end{array}$ & 27 & 9 & $\begin{array}{l}6 \\
0\end{array}$ & $\begin{array}{l}88 \\
143\end{array}$ & \\
\hline $786 \mathrm{~B}$ & $\begin{array}{l}69 R-1 \\
69 R-4\end{array}$ & 68-77 & 98 & & 33 & B series & LCBA & & 0. & & 60 & 0. & 8.3 & 3.3 & 4.13 & o. & & & & 159 & & 121 & 110 & 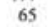 & 21 & III & 2 & 3 & 8 \\
\hline $786 \mathrm{~B}$ & $\begin{array}{l}690-4 \\
69 \mathrm{R}-5\end{array}$ & $60-64$ & $\begin{array}{l}8802.36 \\
803.07\end{array}$ & : & 33 & B series & LCBA & & 0.2 & 12. & 6.4 & 0. & 8. & 4 & 3. & 0 , & & & & & & 123 & & & & & & & \\
\hline $\begin{array}{l}786 \mathrm{~B} \\
786 \mathrm{~B}\end{array}$ & $69 \mathrm{R}-7$ & $34-42$ & 805.68 & : & $\begin{array}{l}33 \\
33\end{array}$ & $\begin{array}{l}\text { LCB seriese } \\
\text { LCB series }\end{array}$ & $\begin{array}{l}\text { LCBA } \\
\text { LCBA }\end{array}$ & & $\begin{array}{l}0.221 \\
0.22\end{array}$ & $\begin{array}{l}111 . \\
12 .\end{array}$ & $\begin{array}{l}7.3 \\
6.9\end{array}$ & 0.1 & $\begin{array}{l}8 . \\
9\end{array}$ & 3. & 3. & $\begin{array}{l}0.1 \\
0\end{array}$ & & & & 2 & & 124 & 70 & 199 & ${ }_{28}^{27}$ & 10 & I & $\begin{array}{l}0 \\
4\end{array}$ & \\
\hline $\begin{array}{l}7866 \mathrm{~B} \\
780\end{array}$ & $70 \mathrm{R}-1$ & $68-73$ & 7.58 & & 33 & 8 series & LCBA & os.. & 0.23 & 12. & 6.05 & 0.1 & 8. & 5 & 3. & & & & & 202 & & 148 & 77 & 70 & & & 2 & 7 & 9 \\
\hline $\begin{array}{l}7868 \mathrm{~B} \\
786 \mathrm{~B}\end{array}$ & $\begin{array}{l}70 \mathrm{R}-1 \\
\text { 70R-2 }\end{array}$ & $92-96$ & 7.82 & une & 33 & 8 series & LCBA & 62.4 & 0.2 & 11. & & & 8. & 6. & 3.44 & 0. & & & 10.1 & 196 & & 118 & 88 & 75 & 23 & "I & & & 8 \\
\hline $786 \mathrm{~B}$ & $\begin{array}{l}70 R-2 \\
I I R-1\end{array}$ & & 45 & & 33 & B series & LCBA & 62 & 0.2 & 12. & 6. & 0.1 & 8. & 6. & 3.42 & 0.7 & & & & 19 & & 122 & 65 & 66 & & & & 133 & \\
\hline 7 & $\begin{array}{l}\text { MR-1 } \\
\text { IRR-1 }\end{array}$ & $\begin{array}{l}1032-108 \\
122-126\end{array}$ & .52 & & $\frac{4}{4}$ & $\begin{array}{l}8 \text { series } \\
\text { series }\end{array}$ & $\begin{array}{l}\text { ICB } \\
\text { ICB }\end{array}$ & & $\begin{array}{l}0 . \\
0 .\end{array}$ & & 7.4 & & & & 2. & & & & & & & 3 & 五 & 41 & $\begin{array}{l}32 \\
32\end{array}$ & 12 & $\begin{array}{l}5 \\
5 \\
\end{array}$ & & \\
\hline 7866B & IIR-1 & $122-126$ & 257.52 & & 4 & $B$ series & ICB & & 0.22 & 13 & 8.03 & 0 & & 6. & 2 & 0. & & & & 159 & & . & 71 & 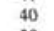 & & & & 0 & \\
\hline $786 \mathrm{~B}$ & $\begin{array}{l}12 R \\
5 R R\end{array}$ & & & & & 3 series & ICB & & 0.16 & & 8.40 & & & 7. & 2. & & & & & & & & 76 & 38 & & & & & \\
\hline & $\begin{array}{l}S \mathrm{SiR} \\
51 \mathrm{R}\end{array}$ & & & & ${ }_{26}^{26}$ & & ICH & & & 12 & 8. & & & 7 & 2 & & & & & & & & & & 31 & 11 & & & \\
\hline $\begin{array}{l}7866 \\
78654 \\
7\end{array}$ & $5 \mid \mathrm{R}=2$ & & & & ${ }_{26}^{20}$ & es & ICB & & & & & & & & & & & & & & & & 10 & & 33 & $\|$ & & & \\
\hline $886 \mathrm{~B}$ & & 50 & 70 & u & 26 & 8 & ICB & & 0 & 12 & 7.9 & 0.1 & & 7. & 2. & & & & & 2 & & 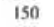 & 56 & 53 & 29 & $\because$ & 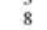 & & \\
\hline 6 & $54 R$ & & 3.54 & 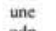 & 26 & es & ICB & & 0. & 12 & 8. & & & 7. & 2. & 0 & & & & & & 9 & z & 40 & 33 & 11 & & & \\
\hline & 5 & & & & 32 & & & & & & & & & & & & & & & & & & & & & & & & \\
\hline & & & & & & & & & & & & & & & & & & & & & & & & & & & & & \\
\hline 786 & 54 & & & 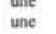 & 26 & & & & & & & & & & 2 & & & & & & & & & & & & & & \\
\hline 786 & 54 & 144 & & u & 26 & & & & & & & & & & & & & & & & & & & & & 9 & & & \\
\hline & & & & & 26 & & & & & & 8. & & & & & & & & & & & & & & & & & & \\
\hline 786 & $\begin{array}{l}54 \mathrm{R} \\
54 \mathrm{R}\end{array}$ & & & u & ${ }_{26}^{26}$ & & & & & & & & & & & & & & & & & & & & & & & & \\
\hline $\begin{array}{l}786 \\
786\end{array}$ & $\begin{array}{l}54 \mathrm{R} \\
\mathrm{IR}\end{array}$ & & & 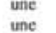 & $\begin{aligned} 26 \\
1\end{aligned}$ & & & & & & & & & & & & & & & 163 & & 138 & 00 & 114 & & & 10 & 134 & \\
\hline 786 & IR & & & & 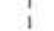 & & & & & & 7. & & & & & & & & & & & & & & & & & & \\
\hline & is & & & & 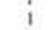 & & & & & & & & & & & & & & & & 374 & 177 & 55 & 67 & & & & 174 & \\
\hline 764 & IR & & & ut & i & & & & & & & & & & & & & & & & & & 58. & & & 9 & & & \\
\hline & 25 & & & o & I & & & & & & & & & & & & & & & & & & & & & & & & \\
\hline & & & & u & 1 & & & & & & & & & & & & & & & & & & & & 27 & 8 & & & \\
\hline 78 & ${ }_{4 \mathrm{R}}^{3 \mathrm{R}}$ & & & ur & I & & & & & & 7 & & & & & & & & & & & & & & & 9 & & & \\
\hline 786 & $\begin{array}{l}4 R \\
3 R\end{array}$ & & & of & 1 & & & & & & & & & & & & & & & & & & & & & & & & \\
\hline $78 t$ & $3 R$ & & & & 1 & & & & & & & & & & & & & & & 103 & & 700 & & & & & 23 & 153 & \\
\hline & & & & wit & i & & & & & & & & & & & & & & & & & & & & & & & & \\
\hline & & & & o & & & & & & & & & & & & & & & & & & & & 74 & & 12 & & & \\
\hline & & & & u & 4 & & & & & & 7. & & & & & & & & & & & & & & & & & & \\
\hline & & & & ur & 4 & & & & & & & & & & & & & & & & & & & & & & & & \\
\hline & 28 & & & of & 13 & & & & & & & & & & & & & & & & & & & & & & & & \\
\hline 7 & 30 & & & w & ${ }_{13}^{13}$ & & & & & & & & & & & & & & & & & & & & & & & & \\
\hline 788 & & & & 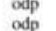 & 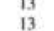 & & & & & & & & & & & & & & & & & & & & & & & & \\
\hline 78 & & & & & 1 & & & & & & & & & & & & & & & & & & & & & & & & \\
\hline & & & & & i. & & & & & & & & & & & & & & & & & & & 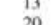 & 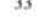 & & & & \\
\hline $78 t$ & 31 & & & u & 13 & & & & & & & & & & & & & & & & & & & 29 & 20 & 11 & 10 & & \\
\hline 86 & & & & 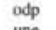 & 2 & & & & & & & & & & & & & & & 149 & & 5 & 68 & 20 & & & 13 & & \\
\hline & & & & ur & ${ }_{24}^{24}$ & & & & & & & & & & & & & & & 88 & & 1 & 52 & 39 & 27 & 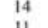 & 9 & 1 & \\
\hline & & 5 & & uit & ${ }_{24}^{24}$ & & & & & & 6 & & & & & & & & & & & & & & & & & & \\
\hline & $43 R \cdot 2$ & $43-52$ & 566.73 & une & 24 & CB series & ICBA & 59.94 & 0.22 & 14.61 & 6.43 & 0.10 & 6.00 & 8.40 & & & & 000 & (II) & & & & & & & & & 140 & \\
\hline
\end{tabular}


Table 2 (continued).

\begin{tabular}{|c|c|c|c|c|c|c|c|c|c|c|c|c|c|c|c|c|c|c|c|c|c|c|c|c|c|c|c|c|c|c|c|}
\hline Hole & Core & $\begin{array}{c}\text { Interval } \\
(\mathrm{cm})\end{array}$ & $\begin{array}{c}\text { Depth } \\
\text { (mbar) }\end{array}$ & Lab & $\begin{array}{l}\text { Lith. } \\
\text { unit }\end{array}$ & $\begin{array}{c}\text { Magma } \\
\text { series }\end{array}$ & $\begin{array}{c}\text { Chemical } \\
\text { group }\end{array}$ & $\mathrm{SiO}_{2}$ & $\mathrm{no}_{2}$ & $\mathrm{Al}_{2} \mathrm{O}_{3}$ & $\mathrm{Fe}_{2} \mathrm{O}_{3}$ & $\mathrm{MnO}$ & $\mathrm{MgO}$ & $\mathrm{CaO}$. & $\mathrm{Na}_{2} \mathrm{O}$ & $\mathrm{K}_{2} \mathrm{O}$ & $\mathrm{P}_{2} \mathrm{O}_{5}$ & $s$ & LOI & $\mathrm{Ni}$ & Cr & v & $\mathrm{Zn}$ & $\mathrm{Cu}_{\mathrm{u}}$ & Sc & Ga & $\mathrm{Rb}$ & Sr & $Y$ & $\mathrm{Zr}$ & $\mathrm{Ba}$ \\
\hline & $45 R-1$ & $41-49$ & 584.71 & une & 24 & ICB series & ICBA & 61.58 & 0.33 & 14.90 & 7.38 & 0.11 & 4.82 & 6.73 & 3.45 & 0.67 & 0.04 & 0.00 & 2.52 & 56 & 193 & 228 & 62 & 100 & 24 & 13 & 10 & 172 & 9 & 45 & 68 \\
\hline & & $39-43$ & & $\begin{array}{l}\text { une } \\
\text { une }\end{array}$ & 24 & Dero & ICBA & & 0.41 & 15,32 & $75^{\circ}$ & & 5.49 & & 3.48 & 0.7 & 0.06 & 0.01 & 4.52 & 61 & 178 & 264 & $\begin{array}{l}02 \\
62\end{array}$ & 76 & 27 & 13 & 9 & 77 & 13 & 46 & 46 \\
\hline $886 \mathrm{~B}$ & $46 \mathrm{R}-1$ & $119-125$ & 595.09 & $\begin{array}{l}\text { unce } \\
\text { une }\end{array}$ & 24 & ICB S & IC & & 0. & 15 & 751 & 0 & 5.32 & $\begin{array}{l}0.91 \\
6.95\end{array}$ & 3.10 & 0.89 & 0.10 & 0.01 & 2.91 & 60 & 185 & 242 & 61 & 89 & 23 & 13 & 10 & 178 & 10 & 47 & 52 \\
\hline $6 \mathrm{~B}$ & $46 \mathrm{R}-2$ & $18=0$ & & une & 24 & is & ic & & 0 . & & 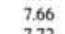 & & 6.26 & 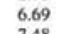 & 3.09 & 0.60 & 0.04 & 0.01 & 6.70 & 65 & 183 & 236 & 66 & 73 & 28 & 13 & 9 & 70 & 10 & 45 & 24 \\
\hline $\begin{array}{l}786 \mathrm{~B} \\
776 \mathrm{~B}\end{array}$ & $\begin{array}{l}47 \mathrm{R}-1 \\
47 \mathrm{R}-1\end{array}$ & ${ }_{112-22}^{18-6}$ & & $\begin{array}{l}\text { une } \\
\text { une }\end{array}$ & $\begin{array}{l}24 \\
24\end{array}$ & $\begin{array}{l}\text { ICB series } \\
\text { ICB serieg }\end{array}$ & $\begin{array}{l}\text { ICBA } \\
\text { ICBA }\end{array}$ & & 0 & & & 0 & $\begin{array}{l}6.04 \\
728\end{array}$ & 7. & $\begin{array}{l}3.09 \\
3.06\end{array}$ & 0.64 & 0.04 & 0.01 & $\begin{array}{r}4.50 \\
\end{array}$ & 70 & 247 & 215 & 65 & 68 & 26 & 14 & $\stackrel{8}{8}$ & 73 & 8 & 41 & 65 \\
\hline $786 \mathrm{~B}$ & $\begin{array}{l}47 \mathrm{R}-1 \\
49 \mathrm{R}-2\end{array}$ & $\begin{array}{l}11212-116 \\
92-103\end{array}$ & $\begin{array}{l}600.42 \\
624.95\end{array}$ & $\begin{array}{l}\text { une } \\
\text { une }\end{array}$ & $\begin{array}{l}24 \\
24\end{array}$ & $\begin{array}{l}\text { ICB series } \\
\text { ICB series }\end{array}$ & $\begin{array}{l}\text { ICBA } \\
\text { ICBA }\end{array}$ & & $\begin{array}{l}0.34 \\
0.41\end{array}$ & & 10 & 8 & $\begin{array}{l}7.28 \\
709\end{array}$ & 6. & $\begin{array}{l}3.06 \\
3.20\end{array}$ & $\begin{array}{l}0.98 \\
0.95\end{array}$ & $\begin{array}{l}0.02 \\
0.06\end{array}$ & $\begin{array}{l}0.02 \\
0.02\end{array}$ & $\begin{array}{l}11.20 \\
10.00\end{array}$ & $\begin{array}{l}72 \\
90\end{array}$ & $\begin{array}{l}230 \\
289\end{array}$ & $\begin{array}{l}118 \\
119\end{array}$ & $\begin{array}{l}60 \\
64\end{array}$ & $\begin{array}{l}93 \\
88\end{array}$ & 29 & 13 & $\begin{array}{l}15 \\
15\end{array}$ & 188 & $\begin{array}{l}5 \\
8\end{array}$ & $\begin{array}{l}41 \\
42\end{array}$ & 27 \\
\hline $6 \mathrm{~B}$ & & $\mid 37-141$ & & une & 24 & & ICBA & & 0.41 & & 8 & & $\begin{array}{l}7.09 \\
7.26\end{array}$ & 6.8 & 3.20 & $\begin{array}{l}0.95 \\
0.58\end{array}$ & $\begin{array}{l}0.00 \\
0.04\end{array}$ & 0.02 & $\begin{array}{r}10.00 \\
6.76\end{array}$ & & 189 & 179 & ${ }_{\infty}^{\infty}$ & 112 & ${ }_{23}^{23}$ & $\begin{array}{l}13 \\
12\end{array}$ & 9 & 50 & & $\begin{array}{l}42 \\
40\end{array}$ & ${ }^{41}$ \\
\hline & 50R-2 & 40,40 & & une & 24 & ICB & & & 0.32 & & 31 & 0 & 9.06 & 6.43 & 297 & $\begin{array}{l}0.52 \\
0.52\end{array}$ & 0.05 & $\begin{array}{l}0.02 \\
0.01\end{array}$ & $\begin{array}{l}0.70 \\
3.74\end{array}$ & & & 169 & 60 & 70 & 25 & 11 & 9 & & 6 & $\begin{array}{l}40 \\
38\end{array}$ & 62 \\
\hline & 53 & $82-8$. & 6 & une & 26 & cries & & & 078 & & 7.67 & 0 & 8.80 & & 2.47 & 0.52 & 0.06 & 0.01 & 4.35 & & 799 & 186 & 60 & 57 & 32 & 13 & 8 & & 7 & 34 & 38 \\
\hline & & $87-9$ & & une & 26 & & ic & & 0.34 & 12.81 & 8.32 & 0.27 & 7.98 & 7.69 & 2.44 & 0.62 & 0.05 & 0.02 & 5.31 & & 694 & 180 & 58 & 74 & 31 & 11 & II & 48 & 6 & 34 & 172 \\
\hline $\begin{array}{l}7868 \mathrm{~B} \\
786 \mathrm{~B}\end{array}$ & $\begin{array}{l}5 S R \cdot 2 \\
71 R-2\end{array}$ & $\begin{array}{l}11-16 \\
141-148\end{array}$ & & une & $\begin{array}{l}26 \\
33\end{array}$ & ICB series & IC & & 0. & & 7 & 0 & 8.25 & 7. & 2.38 & 0.57 & 0.04 & 0 & 6. & & & 9 & 59 & 47 & 25 & 11 & 10 & 14 & 5 & 36 & 35 \\
\hline $\begin{array}{l}7868 \mathrm{~B} \\
786 \mathrm{~B}\end{array}$ & $7 \mid R-4$ & $\begin{array}{l}1 \\
7-121 \\
7.12\end{array}$ & & $\begin{array}{l}\text { whe } \\
\text { une }\end{array}$ & 33 & $\begin{array}{l}8 \text { series } \\
\text { series }\end{array}$ & II & & 0. & & 7.36 & & $\begin{array}{l}7.13 \\
6.37\end{array}$ & 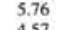 & 3.38 & 0.09 & 0 & & & & & & 101 & 04 & 32 & 10 & 0 & & & 38 & 25 \\
\hline (2000 & $7 \mid R-4$ & $74-80$ & & une & 33 & $\begin{array}{l}\text { ICB series } \\
\text { ICB series }\end{array}$ & ICBA & & 0. & & & $\stackrel{0}{0}$ & $\begin{array}{l}6.37 \\
7.28\end{array}$ & 4. & 3.04 & 0.43 & $\begin{array}{l}0.03 \\
0.03\end{array}$ & 0 & 6 & & & & 107 & 7 & 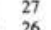 & & & & & 38 & 58 \\
\hline 7868 & $72 \mathrm{R}-1$ & 3. & & une & 34 & ICB series & ICBA & $\begin{array}{l}62.03 \\
62.42\end{array}$ & $\begin{array}{l}0.24 \\
0.33\end{array}$ & & $\begin{array}{l}6.411 \\
7.28\end{array}$ & $\begin{array}{l}0.06 \\
0.06\end{array}$ & $\begin{array}{l}7.28 \\
8.47\end{array}$ & $\begin{array}{l}5.49 \\
1.53\end{array}$ & $\begin{array}{l}3.41 \\
3.28\end{array}$ & $\begin{array}{l}0.11 \\
0.95\end{array}$ & $\begin{array}{l}0.03 \\
0.06\end{array}$ & $\begin{array}{l}0 . \\
2\end{array}$ & $\begin{array}{l}6 \\
7\end{array}$ & & 8 & & 139 & 22 & & & 8 & & 7 & $\begin{array}{l}38 \\
37\end{array}$ & ${ }_{66}^{26}$ \\
\hline & $8 R-1$ & 43-44 & & une & 3 & ADR series & AND & & 03 & & 7.76 & 0 & 4.34 & & & 0.63 & 0.03 & & 5 & & & 182 & & & & & & & & & \\
\hline 786 & $8 R-1$ & $45-$ & & odp & 3 & ADR series & Al & & 0. & & & 0 & 4.15 & & 3.61 & 0.68 & 0 & 0 & 2. & 4 & & & 73 & & & & & & 6 & 51 & \\
\hline $\begin{array}{l}7786 \mathrm{~B} \\
786 \mathrm{~B}\end{array}$ & $\begin{array}{l}9 \mathrm{R}-1 \\
9 \mathrm{R}-1\end{array}$ & $\begin{array}{l}10 \\
15\end{array}$ & & $\begin{array}{c}\text { odp } \\
\text { upe }\end{array}$ & 3 & A & $\begin{array}{l}\text { AND } \\
\text { AND }\end{array}$ & & & & & $\stackrel{0}{0}$ & $\begin{array}{l}4.03 \\
461\end{array}$ & & & 0.60 & 0. & 0. & 1.9 & 40 & & 239 & 72 & 101 & & & & 2 & 10 & 53 & 40 \\
\hline $6 \mathrm{~B}$ & $\begin{array}{c}9 R-1 \\
15 R \cdot 2\end{array}$ & $15-17$ & 25 & une & $\begin{array}{l}3 \\
5\end{array}$ & $\begin{array}{l}\text { R serics } \\
\text { Reries }\end{array}$ & AND & & & & 7.93 & & 4.61 & & 38 & 0.48 & & 0 & 4 & & & & & & & & & & & & \\
\hline $786 \mathrm{~B}$ & $\begin{array}{l}15 R-2 \\
16 R-2\end{array}$ & $\begin{array}{c}32-17 \\
12-17\end{array}$ & & 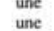 & 5 & $\begin{array}{l}\text { DKs } \\
\text { DRs }\end{array}$ & AND & & 32 & & & 0 & & & 53 & $\begin{array}{l}077 \\
076\end{array}$ & $0_{0}^{0}$ & 0 & 6 & 17 & & & 78 & 75 & & 16 & 7 & & 12 & 56 & 42 \\
\hline $786 \mathrm{~B}$ & $17 \mathrm{R}-1$ & $67-69$ & & 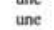 & 5 & ADR series & AND & 60.28 & 32 & 17.72 & 7 & 0 & 2.04 & 0.56 & 4.47 & 0.51 & & 0.02 & 2. & 17 & 15 & 205 & 80 & 80 & 28 & 14 & 9 & 217 & 9 & 57 & 39 \\
\hline 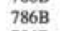 & $19 R-1$ & $10-17$ & & une & 6 & $\mathrm{ADR}$ series & AND & & 0.29 & 17.35 & 7.70 & 0.1 & 4.31 & 7.57 & 3.17 & 0.65 & 0. & 0 & 4.3 & 38 & 6 & & $\begin{array}{l}76 \\
79\end{array}$ & 82 & 27. & 13 & & 210 & & & ${ }_{20}^{\pi}$ \\
\hline $786 \mathrm{~B}$ & & & & odp & 6 & & & & & & & & & & 3.13 & 0.73 & & & & & & & 72 & 82 & & & & & & & ${ }_{31}^{20}$ \\
\hline $786 \mathrm{~B}$ & 20R-1 & $124-128$ & & une & 6 & ADR series & A) & & 0.3 & & $?$ & 0. & 2.51 & & 3.94 & 0.50 & 0 & 0 & 4. & & & & 72 & 83 & 29 & 13 & 6 & & 21 & 57 & $\begin{array}{ll}31 \\
61\end{array}$ \\
\hline $\begin{array}{l}7868 \mathrm{~B} \\
786 \mathrm{~B}\end{array}$ & $\begin{array}{l}2 I R-1 \\
2 \mid R-1\end{array}$ & $\begin{array}{l}26-29 \\
26-30\end{array}$ & 35 & $\begin{array}{c}\text { odp } \\
\text { une }\end{array}$ & $\begin{array}{l}6 \\
6\end{array}$ & $\begin{array}{l}\text { ADR series } \\
A D R \text { seres }\end{array}$ & AN & 59.82 & 0. & 17.84 & 8 & 0. & 1 & & 189 & 0.89 & 0.14 & 0 & & & & & 86 & & & & & & & 58 & $\begin{array}{l}27 \\
27\end{array}$ \\
\hline $786 \mathrm{~B}$ & $\begin{array}{l}211 . \\
37 \mathrm{R}\end{array}$ & $\begin{array}{l}26-30 \\
45-49\end{array}$ & & $\begin{array}{l}\text { une } \\
\text { dep }\end{array}$ & ${ }_{22}^{6}$ & es & & & & & & & & & 4.33 & 0 & 0. & & 5 & $i$ & 17 & & 59 & 78 & 21 & 16 & & & 28 & 56 & 54 \\
\hline 7868 & & $6-8$ & & $\begin{array}{l}\text { cop } \\
\text { odp }\end{array}$ & ${ }_{22}^{22}$ & ies & & & & & & & & & $\begin{array}{l}2.88 \\
2.96\end{array}$ & i & & & 5 & i & & & 61 & $\begin{array}{l}94 \\
66\end{array}$ & & & & & 9 & 44 & $\begin{array}{l}108 \\
54\end{array}$ \\
\hline $\begin{array}{l}786 \mathrm{~B} \\
786 \mathrm{~B}\end{array}$ & $\begin{array}{l}38 \\
30\end{array}$ & $76-83$ & & & 22 & DR series & As & & .26 & & & 0 & & & 49 & 0.16 & & & 8. & 53 & 10 & & 58 & 84 & 16 & 13 & & & 6 & 43 & $\begin{array}{l}54 \\
58\end{array}$ \\
\hline $\begin{array}{l}7868 \mathrm{~B} \\
786 \mathrm{~B}\end{array}$ & $\begin{array}{l}39 \mathrm{R}-1 \\
39 \mathrm{R}-2\end{array}$ & $47-5$ & & une & 22 & DR series & AND & 6200 & 0.28 & 17.16 & 6 & 0 & 2.44 & 7.1 & 3.85 & 0.44 & 0. & & 2. & 14 & iI & 38 & 112 & 32 & 24 & 14 & & & II & 47 & 53 \\
\hline & $\begin{array}{l}39 \mathrm{R}-2 \\
49 \mathrm{R}-4\end{array}$ & ${ }^{119-123}$ & & ne & 22 & ADR series & Af & 62.75 & & & & 0 & 2. & & 3.5 & & & & & 1 & 11 & & 67 & 108 & 26 & & & & II & & 54 \\
\hline & 651 & $\begin{array}{l}32 \\
21\end{array}$ & 61 & $\begin{array}{c}\text { opp } \\
\text { upe }\end{array}$ & $\begin{array}{l}24 \\
30\end{array}$ & $\begin{array}{l}\text { ADR seres ses } \\
A D R \text { series }\end{array}$ & $\hat{A}$ & & & & & & & & & $0_{0}^{0}$ & & & 4. & 39 & $\begin{array}{l}93 \\
18\end{array}$ & & 58 & 67 & 29 & 15 & & 0 & 11 & 45 & $\begin{array}{l}46 \\
56\end{array}$ \\
\hline & 16 & $135-138$ & & une & 5 & ADR series & D & & 0 & & & & & & 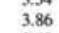 & 0.69 & 0 & & 3.43 & & & & & & & & & & & & \\
\hline & & $137-1$ & & ddp & 5 & $\mathrm{ADR}$ & D & & & & & & & & & 0 & 0 & & 2 & 9 & 6 & 9 & 65 & 91 & & & 7 & 7 & 10 & 57 & 35 \\
\hline & & & & odp & II & & & & & & & & & & & & & & & , & 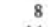 & & . & & & & & & 14 & & 84 \\
\hline & 24 & $4 !$ & & une & i1 & & D & & & & & & & & & 1 & & & 1 & 7 & 14 & & $\begin{array}{l}49 \\
55\end{array}$ & 49 & 19 & 15 & 13 & & is & 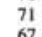 & 80 \\
\hline & $\begin{array}{l}24 R-2 \\
25 R-1\end{array}$ & $\begin{array}{c}5-9 \\
6472\end{array}$ & & $\begin{array}{l}\text { une } \\
\text { une }\end{array}$ & 临 & $\begin{array}{l}\text { ADR series } \\
A D R \text { seres }\end{array}$ & ${ }_{\mathrm{D}}^{\mathrm{D}}$ & & 0 & & & & $i$ & & $\begin{array}{l}4.44 \\
4.88\end{array}$ & 1 & a. & & & $\begin{array}{l}8 \\
6\end{array}$ & & & $\begin{array}{l}55 \\
48\end{array}$ & 40 & 13 & 14 & & & 10 & & 68 \\
\hline 78 & & 647 & & odp & i1 & & & & & & & & & & & & & & & & & & 48 & & & & & 95 & $\begin{array}{l}12 \\
12\end{array}$ & & 73 \\
\hline & & & & & 11 & & & & & & & & & & & & & & & & & & & & & & & & & & \\
\hline 786 & 35 & & & a & 20 & $A$ & & & & & & & & & & & & & & & & & & & & & & & & & \\
\hline 786 & & & & & ${ }^{20}$ & A & & & & & & & & & & & & & & & & & & & 16 & 1 & & & & & 80 \\
\hline $\begin{array}{l}788 \mathrm{~B} \\
7808\end{array}$ & & & & & 20 & & & & & & & & & & & & & & & & & & & & & & & & 0 & & 53 \\
\hline & & & & & 8 & & & & & & & & & & & & & & & & & & & & & & & & 2 & & 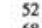 \\
\hline 70 & & & & odp & 8 & & & & & & & & & & & & & & & & & & & & 15 & & & & & & 88 \\
\hline $786 \mathrm{~B}$ & & & & & 16 & & & & & & & & & & & & & & & & & & & & & & & & & & 58 \\
\hline & & & & & 20 & & & & & & & & & & & & & & & & & & & & 12 & & & & & 4 & 80 \\
\hline 786 & 6 & & & & 28 & & & & & & & & & & & & & & & & & & & 32 & 12 & & & & & 1 & 175 \\
\hline & & & & & 28 & & & & & & & & & & & & & & & & & & & & & & & & & & 91 \\
\hline & & & & & 28 & & & & & & & & & & & & & & & & & & & & & & & & & 32 & 93 \\
\hline & & & & & & & & & & & & & & & & & & & & & & & & & & & & & & & 85 \\
\hline & & & & & 29 & & & & & & & & & & & & & & & & & & & & 13 & & & & 0 & 1 & 679 \\
\hline & & & & & 29 & & & & & & & & & & & & & & & & & & & & & & & & & & $\begin{array}{l}56 \\
73\end{array}$ \\
\hline & & & & & 29 & & & & & & & & & & & & & & & & & & & & 16 & & & & 0 & & 25 \\
\hline 788 & 6 & & & & 29 & & & & & & & & & & & & & & & & & & & & & & & & & & 32 \\
\hline 786 & & & & u & 29 & ADR series & & & & & & & & & & & & & & & & & & & & 10 & & & & & 08 \\
\hline & 67 & & & u & 29 & ADR series & & & & & & & & & & & & & & & & & 105 & & 14 & 8 & & & 2 & 7 & 30 \\
\hline 7860A & $\frac{12}{60}$ & $\begin{array}{r}140 \\
24\end{array}$ & & & 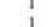 & & & & & & & & & & & & & & & & 320 & & & & & & & & 6 & 6 & 36 \\
\hline & & & & & 1 & & & & & & & & & & & & & & & & & & ${ }_{73}^{113}$ & & & & & & ${ }_{4}^{4}$ & & $\begin{array}{l}77 \\
8\end{array}$ \\
\hline & & 17 & & & i & & & & & & & & & & & & & & & & & & & & & & & & & & II \\
\hline & $13 x-C 0$ & 27.29 & 115.57 & odp & 1 & & misc. & 53. & 0.24 & 12.9 & 8.41 & 0.01 & 13.58 & 5.8 & 2.34 & 0.8 & 0.4 & 0.0 & 5.70 & 268 & 613 & 143 & 63 & 52 & & & 13 & 100 & 6 & & ${ }_{23}$ \\
\hline
\end{tabular}


Table 3. ICP-MS trace element data. Samples crushed in tungsten carbide are indicated by the letters WC in the Ta column.

\begin{tabular}{|c|c|c|c|c|c|c|c|c|c|c|c|c|c|c|c|c|c|c|}
\hline Core & $\begin{array}{c}\text { Interval } \\
(\mathrm{cm})\end{array}$ & $\begin{array}{c}\text { Chemical } \\
\text { group }\end{array}$ & & $\mathrm{Sc}$ & $\mathrm{Ti}$ & V & $\mathrm{Mn}$ & Co & $\mathrm{Ni}$ & $\mathrm{Cu}$ & $\mathrm{Rb}$ & $\mathrm{Sr}$ & $\mathrm{Y}$ & $\mathrm{Zr}$ & $\mathrm{Nb}$ & Cs & $\mathrm{Ba}$ & La \\
\hline $05 R-2$ & $70-72$ & HCB & late dykes & 25 & & & & 37 & 143 & 47 & 6.7 & 141 & 11.1 & 34.3 & 0.58 & 0.56 & 16.7 & 2.00 \\
\hline 05R-2 & $69-71$ & HCB & late dykes & 35 & 0.37 & 208 & 0.13 & 41 & 179 & 36 & 5.5 & 157 & 11.0 & 32.2 & 0.39 & 0.19 & 17.3 & 2.09 \\
\hline 06R-2 & $128-130$ & HCB & late dykes & & & & & & & & 3.9 & 127 & 10.2 & 32.8 & 0.75 & & 9.2 & 1.62 \\
\hline $06 \mathrm{R}-3$ & $17-22$ & НCB & late dykes & 26 & & 213 & & & 154 & 45 & 9.3 & 110 & 9.7 & 27.5 & 0.60 & & 11.6 & 1.53 \\
\hline $21 R-2$ & $72-76$ & НСB & late dykes & 23 & & & & 36 & & & 11.6 & 138 & 8.9 & 26.0 & 0.78 & & 20.0 & 1.82 \\
\hline $21 R-2$ & $103-107$ & HCB & late dykes & 29 & 0.20 & 143 & 0.15 & 44 & 258 & 86 & 5.8 & 139 & 6.8 & 27.4 & 0.25 & 0.20 & 16.7 & 1.45 \\
\hline $34 \mathrm{R}-3$ & $45-47$ & HCB & late dykes & & & & & & & & 14.9 & 138 & 6.5 & 36.1 & 0.60 & & 16.5 & 2.14 \\
\hline $34 \mathrm{R}-4$ & $14-20$ & HCB & late dykes & 33 & 0.23 & 152 & 0.13 & 43 & 273 & 30 & 17.6 & 140 & 6.3 & 28.4 & 0.30 & 1.23 & 16.3 & 1.29 \\
\hline $40 \mathrm{R}-2$ & $83-90$ & HCB & late dykes & 30 & 0.29 & 197 & 0.16 & 47 & 321 & 27 & 9.6 & 143 & 8.1 & 24.2 & 0.42 & 0.34 & 17.3 & 1.34 \\
\hline $20 \mathrm{R}-1$ & $72-74$ & ICB & late dykes & 33 & 0.24 & 173 & 0.19 & 76 & 258 & 40 & 7.4 & 136 & 5.2 & 30.8 & 0.27 & 0.17 & 11.2 & 0.94 \\
\hline $44 \mathrm{R}-1$ & $38-45$ & ICB & late dykes & 28 & 0.29 & 192 & 0.20 & 52 & 395 & 48 & 12.0 & 132 & 8.2 & 26.0 & 0.33 & 0.25 & 16.9 & 1.34 \\
\hline $57 R-2$ & $122-125$ & LCB? & LCB series & 30 & 0.22 & 181 & 0.17 & 41 & 276 & 13 & 21.8 & 144 & 9.5 & 32.8 & 0.66 & 0.27 & 23.1 & 2.44 \\
\hline $57 \mathrm{R}-4$ & $7-13$ & LCB & LCB series & 32 & 0.23 & & 0.14 & & & 22 & 5.8 & 124 & 7.4 & 35.5 & 0.70 & 0.03 & 18.2 & 1.75 \\
\hline $58 \mathrm{R}-1$ & $121-126$ & LCB & LCB series & 28 & 0.20 & 159 & 0.13 & 43 & 299 & 21 & 4.1 & 122 & 7.4 & 31.5 & 0.66 & 0.05 & 12.7 & 2.06 \\
\hline $62 R-3$ & $40-42$ & LCB & LCB series & 29 & 0.20 & & 0.13 & & & 67 & 9.3 & 105 & 7.0 & 34.3 & 0.65 & 0.01 & 46.1 & 1.73 \\
\hline $69 \mathrm{R}-4$ & $105-113$ & LCBA & LCB series & 23 & 0.22 & 164 & 0.12 & 32 & 213 & 95 & 7.6 & 134 & 7.8 & 41.9 & 0.82 & 0.01 & 36.6 & 2.23 \\
\hline $69 \mathrm{R}-7$ & $34-42$ & LCBA & LCB series & 22 & 0.23 & 129 & 0.11 & 33 & 218 & 81 & 2.6 & 122 & 7.1 & 39.8 & 0.63 & 0.02 & 44.8 & 1.85 \\
\hline $70 \mathrm{R}-1$ & $68-73$ & LCBA & LCB series & 20 & & & & 30 & 163 & 70 & 2.0 & 146 & 8.2 & $4+2$ & 0.77 & & 52.4 & 2.62 \\
\hline 70R-2 & $5-11$ & LCBA & LCB series & 17 & & 104 & & & 170 & 51 & 7.3 & 103 & 6.3 & 28.9 & 0.48 & & 58.6 & 2.75 \\
\hline $11 R-1$ & $122-126$ & ICB & ICB series & 25 & & & & 37 & 147 & 30 & 7.2 & 159 & 5.1 & 40.0 & 0.80 & 0.29 & 20.8 & 1.31 \\
\hline $11 \mathrm{R}-1$ & $121-123$ & ICB & ICB series & 35 & 0.27 & 168 & 0.10 & 39 & 209 & 14 & 14.5 & 161 & 10.9 & 30.1 & 0.32 & 0.82 & 18.5 & 1.66 \\
\hline 5IR-1 & $75-79$ & ICB & ICB series & 29 & 0.21 & & 0.14 & & & 51 & 8.2 & 154 & 8.5 & 34.7 & 0.62 & 0.13 & 26.5 & 2.25 \\
\hline 51R-1 & $51-55$ & ICB & ICB series & 26 & & & & 43 & 182 & 30 & 3.6 & 164 & 16.6 & 37.2 & 0.75 & 0.14 & 19.2 & 5.47 \\
\hline $67 R-1$ & $63-65$ & ICB & ICB series & 28 & & & & 42 & 253 & 63 & 3.8 & 135 & 6.1 & 25.7 & 0.43 & 0.11 & 23.7 & 1.16 \\
\hline $67 R-1$ & $56-59$ & ICB & ICB series & 34 & 0.28 & & 0.11 & & & 39 & 6.5 & 147 & 9.0 & 34.0 & 0.44 & 0.07 & 7.6 & 1.69 \\
\hline $01 R-1$ & $61-64$ & ICBA & ICB series & & & & & & & & 6.3 & 174 & 6.2 & 44.4 & & & 36.0 & 1.83 \\
\hline $01 R-1$ & $75-79$ & ICBA & ICB series & 25 & 0.21 & 171 & 0.11 & 29 & 101 & 53 & 13.0 & 165 & 5.6 & 38.6 & 0.59 & 0.16 & 38.6 & 1.69 \\
\hline $02 R-1$ & $72-76$ & ICBA & ICB series & 25 & 0.19 & 146 & 0.12 & 29 & 100 & 63 & 8.6 & 191 & 7.0 & 41.3 & 0.58 & 0.18 & 43.6 & 2.01 \\
\hline $04 \mathrm{R}-1$ & $109-111$ & ICBA & ICB series & 23 & 0.20 & 159 & 0.12 & 27 & 92 & 65 & 10.7 & 186 & 6.6 & 42.1 & 0.69 & 0.20 & 50.1 & 2.17 \\
\hline $05 R-1$ & $23-25$ & ICBA & ICB series & 21 & & 151 & & & 61 & 68 & 12.5 & 173 & 6.1 & 44.0 & 0.72 & & 22.4 & 1.70 \\
\hline $12 \mathrm{R}-2$ & $124-126$ & ICBA & ICB series & 30 & 0.19 & 171 & 0.14 & 35 & 144 & 63 & 9.3 & 188 & 5.3 & 34.9 & 0.62 & 0.12 & 34.8 & 1.82 \\
\hline 13R-2 & $134-138$ & ICBA & ICB series & 24 & 0.19 & 139 & 0.12 & 28 & 123 & 61 & 7.6 & 200 & 5.6 & 38.0 & 0.60 & 0.16 & 40.1 & 3.77 \\
\hline $30 \mathrm{R}-1$ & $30-32$ & ICBA & ICB series & 31 & 0.16 & 146 & 0.11 & 32 & 134 & 23 & 10.4 & 212 & 4.8 & 29.0 & 0.45 & 0.35 & 44.7 & 1.70 \\
\hline $30 \mathrm{R}-1$ & $29-31$ & ICBA & ICB series & & & & & & & & 20.0 & 192 & 5.0 & 33.7 & 0.84 & & 46.4 & 2.46 \\
\hline $37 \mathrm{R}-1$ & $95-98$ & ICBA & ICB series & & & & & & & & 12.3 & 185 & 8.7 & 40.3 & 0.78 & & 27.8 & 2.01 \\
\hline $42 R-4$ & $46-54$ & ICBA & ICB series & 24 & 0.24 & 167 & 0.08 & 24 & 94 & 37 & 12.8 & 227 & 6.8 & 43.6 & 0.73 & 0.18 & 51.4 & 2.46 \\
\hline $43 R-2$ & $43-52$ & ICBA & ICB series & 19 & & 183 & & & 119 & 10 & 12.5 & 166 & 7.1 & 36.2 & 0.61 & & 35.3 & 1.87 \\
\hline $45 R-1$ & $41-47$ & ICBA & ICB series & 25 & 0.34 & 258 & 0.12 & 25 & 66 & 99 & 17.8 & 193 & 9.8 & 44.7 & 0.80 & 0.23 & 57.1 & 2.39 \\
\hline $71 \mathrm{R}-4$ & $141-148$ & ICBA & ICB series & 27 & 0.26 & 203 & 0.08 & 27 & 113 & 90 & 6.8 & 178 & 7.6 & 34.3 & 0.66 & 0.06 & 27.1 & 2.04 \\
\hline $71 \mathrm{R}-4$ & $7-12$ & ICBA & ICB series & 25 & 0.24 & 182 & 0.09 & 28 & 121 & 78 & 5.9 & 150 & 7.6 & 36.4 & 0.76 & 0.06 & 43.7 & 2.36 \\
\hline $72 \mathrm{R}-1$ & $3-7$ & ICBA? & ICB series & 22 & 0.25 & 174 & 0.05 & 22 & 103 & 81 & 14.7 & 141 & 5.0 & 22.0 & 0.69 & 0.33 & 86.7 & 1.74 \\
\hline $08 \mathrm{R}-1$ & $45-47$ & AND & ADR series & 25 & 0.31 & & 0.12 & & & 65 & 10.1 & 218 & 13.1 & 48.7 & 0.82 & 0.11 & 22.3 & 3.02 \\
\hline $09 \mathrm{R}-1$ & $15-17$ & AND & ADR series & 25 & 0.32 & 207 & 0.12 & 28 & 55 & 90 & 7.9 & 234 & 9.6 & 49.0 & 0.71 & 0.22 & 21.1 & 2.34 \\
\hline $15 R-2$ & 3-8 & AND & ADR series & 22 & 0.33 & 214 & 0.12 & 19 & 20 & 77 & 13.2 & 257 & 9.7 & 58.0 & 0.99 & 0.16 & 44.9 & 3.21 \\
\hline $19 \mathrm{R}-1$ & $91-94$ & AND & ADR series & 21 & & & & 34 & 35 & 69 & 7.7 & 215 & 8.0 & 49.9 & 1.26 & 0.32 & 35.0 & 2.59 \\
\hline $21 R-1$ & $20-28$ & AND & ADR series & 22 & 0.31 & 182 & 0.08 & 12 & 17 & 82 & 15.8 & 245 & 23.3 & 57.4 & 0.89 & 0.22 & 49.5 & 7.32 \\
\hline $21 \mathrm{R}-1$ & $26-29$ & AND & ADR series & & & & & & & & 10.8 & 244 & 18.8 & 59.3 & 1.36 & & 35.4 & 6.33 \\
\hline $38 \mathrm{R}-1$ & $76-83$ & AND & ADR series & 19 & 0.26 & 194 & 0.10 & 24 & 28 & 65 & 20.9 & 281 & 7.5 & 42.8 & 0.63 & 0.74 & 82.1 & 2.34 \\
\hline $49 \mathrm{R}-4$ & $32-37$ & AND & ADR series & 25 & & & & 31 & 50 & 87 & 10.9 & 186 & 9.8 & 48.6 & 1.17 & 0.18 & 60.0 & 3.86 \\
\hline $16 \mathrm{R}-1$ & $137-141$ & DAC & ADR series & & & & & & & & 14.1 & 208 & 8.5 & 55.9 & & & 39.4 & 2.69 \\
\hline $25 R-1$ & $64-72$ & DAC & ADR series & 16 & 0.39 & 342 & 0.08 & 11 & 7 & 37 & 37.6 & 207 & 11.8 & 71.5 & 1.13 & 0.16 & 77.2 & 3.78 \\
\hline $35 R-1$ & $75-78$ & DAC & ADR series & 20 & 0.29 & 140 & 0.06 & 14 & 26 & 32 & 10.5 & 232 & 10.0 & 62.3 & 1.03 & 0.16 & 66.4 & 3.13 \\
\hline $35 R-2$ & $122-126$ & DAC & ADR series & & & & & & & & 11.7 & 187 & 9.0 & 60.3 & 1.25 & & 42.7 & 2.51 \\
\hline $21 \mathrm{R}-1$ & $129-131$ & RHY & ADR series & 8 & & & & 12 & 7 & 52 & 28.8 & 134 & 11.0 & 83.7 & 1.34 & 2.13 & 56.5 & 3.36 \\
\hline 21R-2 & $28-32$ & RHY & ADR series & 11 & 0.27 & 63 & 0.09 & 8 & 12 & 94 & 39.0 & 154 & 12.4 & 81.5 & 1.07 & 0.70 & 81.6 & 3.94 \\
\hline $61 R-4$ & $96-102$ & RHY & ADR series & 14 & 0.38 & 103 & 0.10 & 8 & 7 & 40 & 36.7 & 164 & 14.8 & 95.1 & 1.39 & 0.36 & 189 & 4.53 \\
\hline $63 R-2$ & $72-74$ & RHY & ADR series & & & & & & & & 37.0 & 42 & 11.1 & 71.1 & 1.30 & & 486 & 3.63 \\
\hline $66 \mathrm{R}-1$ & $88-90$ & RHY & ADR series & & & & & & & & 32.1 & 96 & 10.3 & 75.8 & 1.30 & 0.23 & 324 & 4.44 \\
\hline $67 \mathrm{R}-1$ & $33-41$ & RHY & ADR series & 8 & & 64 & & & 6 & 35 & 21.0 & 94 & 10.3 & 61.7 & 0.85 & & 286 & 3.06 \\
\hline $12 \mathrm{X}-1$ & $133-138$ & misc. & $786 \mathrm{~A}$ & 33 & 0.41 & 271 & 0.10 & 30 & 88 & 42 & 20.8 & 165 & 5.5 & 44.9 & 0.67 & 0.28 & 18.4 & 0.70 \\
\hline $06 \mathrm{X}-5$ & $24-27$ & misc. & $786 \mathrm{~A}$ & 41 & 0.82 & & 0.18 & & & 147 & 7.6 & 170 & 19.3 & 37.9 & 0.83 & 0.74 & 66.6 & 1.24 \\
\hline $16 \mathrm{X}-\mathrm{CC}$ & $24-29$ & misc. & $786 \mathrm{~A}$ & 31 & 0.24 & 201 & 0.14 & 41 & 277 & 74 & 9.7 & 140 & 6.3 & 27.5 & 0.41 & 0.06 & 25.0 & 1.06 \\
\hline $17 \mathrm{X}-\mathrm{CC}$ & $4-12$ & misc. & $786 \mathrm{~A}$ & 29 & 0.22 & 179 & 0.12 & 45 & 275 & 37 & 24.9 & 50 & 3.8 & 24.7 & 0.42 & 0.32 & 30.8 & 0.52 \\
\hline
\end{tabular}


Table 3 (continued).

\begin{tabular}{|c|c|c|c|c|c|c|c|c|c|c|c|c|c|c|c|c|c|}
\hline $\mathrm{Ce}$ & Pr & $\mathrm{Nd}$ & $\mathrm{Sm}$ & $\mathrm{Eu}$ & Gd & $\mathrm{Tb}$ & Dy & Ho & $\mathrm{Er}$ & $\mathrm{Tm}$ & $\mathrm{Yb}$ & $\mathrm{Lu}$ & $\mathrm{Hf}$ & $\mathrm{Ta}$ & $\mathrm{Pb}$ & $\mathrm{Th}$ & $\mathrm{U}$ \\
\hline 4.15 & 0.70 & 3.87 & 0.96 & 0.31 & 1.14 & 0.19 & 1.44 & 0.31 & 0.96 & 0.15 & 1.03 & 0.17 & 0.98 & WC & 0.69 & 0.14 & 0.16 \\
\hline 4.15 & 0.74 & 4.02 & 1.03 & 0.37 & 1.55 & 0.27 & 1.71 & 0.38 & 1.09 & 0.17 & 1.17 & 0.17 & 0.98 & 0.02 & 1.27 & 0.18 & 0.18 \\
\hline 3.53 & 0.45 & 3.52 & 0.91 & 0.38 & 1.23 & 0.23 & 1.65 & 0.33 & 1.22 & 0.19 & 1.17 & 0.17 & 1.15 & WC & 1.17 & 0.07 & \\
\hline 3.77 & 0.46 & 2.96 & 1.09 & 0.32 & 1.06 & 0.21 & 1.54 & 0.24 & 0.86 & 0.18 & 0.90 & 0.17 & 0.98 & WC & 0.81 & 0.14 & \\
\hline 2.54 & 0.47 & 2.19 & 0.66 & 0.23 & 1.01 & 0.17 & 1.20 & 0.27 & 0.77 & 0.12 & 0.97 & 0.11 & 1.22 & WC & 0.80 & 0.13 & 0.13 \\
\hline 2.86 & 0.45 & 2.17 & 0.49 & 0.21 & 0.89 & 0.17 & 0.98 & 0.27 & 0.71 & 0.14 & 0.83 & 0.12 & 0.83 & 0.01 & 0.77 & 0.19 & 0.16 \\
\hline 3.30 & 0.49 & 2.19 & 0.50 & 0.21 & 0.73 & 0.16 & 0.92 & 0.20 & 0.72 & 0.11 & 0.74 & 0.11 & 0.97 & WC & & & \\
\hline 2.81 & 0.46 & 2.01 & 0.56 & 0.21 & 0.64 & 0.10 & 0.99 & 0.19 & 0.64 & 0.13 & 0.70 & 0.11 & 0.86 & 0.02 & 1.00 & 0.18 & 0.10 \\
\hline 2.98 & 0.56 & 2.76 & 0.68 & 0.29 & 1.16 & 0.17 & 1.30 & 0.30 & 0.84 & 0.16 & 0.88 & 0.14 & 0.72 & 0.02 & 0.87 & 0.14 & 0.12 \\
\hline 2.32 & 0.34 & 1.66 & 0.45 & 0.18 & 0.74 & 0.12 & 0.93 & 0.19 & 0.58 & 0.10 & 0.59 & 0.09 & 0.96 & 0.02 & 1.02 & 0.22 & 0.10 \\
\hline 3.17 & 0.53 & 2.84 & 0.70 & 0.28 & 1.09 & 0.22 & 1.23 & 0.27 & 0.75 & 0.15 & 0.82 & 0.13 & 0.78 & 0.02 & 0.70 & 0.12 & 0.24 \\
\hline 4.50 & 0.69 & 3.65 & 0.78 & 0.33 & 1.30 & 0.19 & 1.30 & 0.31 & 0.86 & 0.14 & 0.90 & 0.14 & 0.99 & 0.05 & 1.86 & 0.29 & 0.24 \\
\hline 3.87 & 0.64 & 3.10 & 0.83 & 0.26 & 1.05 & 0.13 & 1.09 & 0.24 & 0.70 & 0.09 & 0.73 & 0.12 & 0.95 & 0.05 & 1.47 & 0.28 & 0.13 \\
\hline 3.90 & 0.62 & 3.12 & 0.82 & 0.22 & 1.07 & 0.19 & 1.08 & 0.25 & 0.71 & 0.12 & 0.72 & 0.13 & 0.89 & 0.05 & 2.22 & 0.26 & 0.16 \\
\hline 3.70 & 0.61 & 2.85 & 0.73 & 0.26 & 1.04 & 0.12 & 1.02 & 0.23 & 0.66 & 0.09 & 0.80 & 0.15 & 0.99 & WC & 1.53 & 0.26 & 0.20 \\
\hline 5.21 & 0.78 & 3.47 & 0.94 & 0.31 & 1.21 & 0.20 & 1.18 & 0.23 & 0.80 & 0.12 & 0.97 & 0.13 & 1.32 & 0.06 & 3.43 & 0.31 & 0.31 \\
\hline 4.20 & 0.64 & 3.12 & 0.79 & 0.25 & 1.03 & 0.18 & 1.14 & 0.23 & 0.67 & 0.12 & 0.78 & 0.13 & 1.28 & 0.06 & 3.36 & 0.39 & 0.31 \\
\hline 5.52 & 0.80 & 3.65 & 0.97 & 0.34 & 1.20 & 0.21 & 1.21 & 0.27 & 0.77 & 0.14 & 0.92 & 0.15 & 1.25 & WC & 4.26 & 0.33 & 0.27 \\
\hline 5.13 & 0.77 & 3.78 & 1.05 & 0.26 & 1.04 & 0.24 & 1.37 & 0.20 & 0.77 & 0.15 & 0.76 & 0.08 & 1.06 & 0.03 & 1.66 & 0.30 & \\
\hline 3.37 & 0.49 & 2.32 & 0.61 & 0.24 & 0.78 & 0.14 & 0.84 & 0.17 & 0.57 & 0.10 & 0.70 & 0.11 & 1.27 & WC & 2.55 & 0.33 & 0.14 \\
\hline 3.19 & 0.48 & 2.58 & 0.65 & 0.27 & 0.99 & 0.18 & 1.49 & 0.29 & 0.98 & 0.14 & 1.05 & 0.17 & 0.86 & 0.04 & 2.05 & 0.15 & 0.28 \\
\hline 5.18 & 0.74 & 3.46 & 1.00 & 0.37 & 1.32 & 0.19 & 1.27 & 0.26 & 0.83 & 0.11 & 0.86 & 0.14 & 1.03 & 0.04 & 2.00 & 0.34 & 0.15 \\
\hline 12.6 & 1.42 & 5.98 & 1.22 & 0.43 & 1.71 & 0.30 & 1.84 & 0.44 & 1.38 & 0.25 & 1.40 & 0.25 & 1.13 & WC & 1.99 & 0.29 & 0.15 \\
\hline 2.57 & 0.37 & 1.97 & 0.61 & 0.22 & 0.81 & 0.15 & 1.00 & 0.20 & 0.72 & 0.14 & 0.75 & 0.11 & 0.88 & WC & 0.97 & 0.09 & 0.11 \\
\hline 3.66 & 0.59 & 3.07 & 0.91 & 0.36 & 1.31 & 0.20 & 1.49 & 0.31 & 0.89 & 0.12 & 0.93 & 0.16 & 1.00 & 0.04 & 0.95 & 0.22 & 0.18 \\
\hline 3.80 & 0.46 & 2.59 & 0.71 & 0.21 & 0.85 & 0.14 & 0.97 & 0.20 & 0.67 & 0.11 & 0.67 & 0.13 & & WC & 1.53 & 0.32 & 0.15 \\
\hline 3.94 & 0.55 & 2.80 & 0.64 & 0.24 & 0.97 & 0.14 & 0.94 & 0.20 & 0.58 & 0.11 & 0.69 & 0.10 & 1.16 & 0.04 & 1.87 & 0.42 & 0.20 \\
\hline 4.18 & 0.62 & 3.00 & 0.73 & 0.28 & 0.99 & 0.17 & 1.02 & 0.23 & 0.63 & 0.12 & 0.65 & 0.11 & 1.16 & 0.04 & 2.02 & 0.32 & 0.22 \\
\hline 4.56 & 0.68 & 3.26 & 0.72 & 0.23 & 1.07 & 0.17 & 1.05 & 0.27 & 0.64 & 0.11 & 0.75 & 0.13 & 1.28 & 0.05 & 3.15 & 0.39 & 0.24 \\
\hline 4.52 & 0.67 & 3.69 & 0.95 & 0.37 & 1.11 & 0.16 & 1.05 & 0.17 & 0.57 & 0.06 & 0.59 & 0.10 & 1.55 & 0.09 & 1.90 & 0.28 & \\
\hline 3.59 & 0.55 & 2.66 & 0.73 & 0.22 & 0.90 & 0.15 & 0.81 & 0.21 & 0.53 & 0.08 & 0.58 & 0.11 & 1.00 & 0.08 & 1.87 & 0.35 & 0.16 \\
\hline 3.72 & 0.56 & 2.58 & 0.60 & 0.22 & 0.79 & 0.13 & 0.84 & 0.20 & 0.61 & 0.10 & 0.60 & 0.09 & 1.16 & 0.06 & 1.89 & 0.36 & 0.21 \\
\hline 3.34 & 0.45 & 2.27 & 0.59 & 0.21 & 0.78 & 0.12 & 0.77 & 0.15 & 0.47 & 0.08 & 0.52 & 0.08 & 0.86 & 0.05 & 1.76 & 0.33 & 0.24 \\
\hline 3.35 & 0.50 & 2.19 & 0.64 & 0.21 & 0.79 & 0.15 & 0.80 & 0.18 & 0.48 & 0.08 & 0.54 & 0.10 & 1.07 & WC & 1.76 & 0.20 & 0.30 \\
\hline 3.83 & 0.56 & 2.71 & 0.77 & 0.27 & 1.05 & 0.20 & 1.25 & 0.30 & 0.89 & 0.15 & 0.91 & 0.16 & 1.29 & wC & & & \\
\hline 4.91 & 0.68 & 3.31 & 0.69 & 0.32 & 1.09 & 0.15 & 1.01 & 0.22 & 0.66 & 0.09 & 0.79 & 0.13 & 1.12 & 0.06 & 4.08 & 0.43 & 0.23 \\
\hline 3.81 & 0.60 & 2.93 & 0.82 & 0.20 & 0.95 & 0.13 & 0.98 & 0.18 & 0.66 & 0.17 & 0.73 & 0.11 & 0.92 & 0.03 & 1.41 & 0.26 & \\
\hline 5.25 & 0.80 & 3.97 & 1.06 & 0.36 & 1.46 & 0.25 & 1.58 & 0.37 & 0.99 & 0.15 & 1.12 & 0.15 & 1.35 & 0.07 & 2.67 & 0.35 & 0.24 \\
\hline 4.58 & 0.69 & 3.53 & 0.82 & 0.30 & 0.99 & 0.17 & 1.25 & 0.25 & 0.77 & 0.13 & 0.78 & 0.11 & 1.01 & 0.08 & 1.82 & 0.28 & 0.45 \\
\hline 5.43 & 0.80 & 3.87 & 0.95 & 0.29 & 1.29 & 0.20 & 1.20 & 0.26 & 0.77 & 0.13 & 0.79 & 0.12 & 1.04 & 0.08 & 3.00 & 0.27 & 0.50 \\
\hline 3.92 & 0.52 & 2.65 & 0.66 & 0.23 & 0.61 & 0.10 & 0.83 & 0.16 & 0.50 & 0.06 & 0.50 & 0.07 & 0.58 & 0.05 & 0.43 & 0.15 & 0.08 \\
\hline 6.98 & 1.48 & 7.15 & 2.24 & 0.76 & 2.52 & 0.38 & 2.52 & 0.53 & 1.35 & 0.21 & 1.30 & 0.21 & 1.37 & WC & 2.13 & 0.44 & 0.12 \\
\hline 4.71 & 1.09 & 4.74 & 1.28 & 0.42 & 1.55 & 0.27 & 1.67 & 0.38 & 1.06 & 0.17 & 1.06 & 0.14 & 1.55 & 0.03 & 2.37 & 0.46 & 0.09 \\
\hline 7.35 & 1.02 & 5.50 & 1.27 & 0.44 & 1.75 & 0.30 & 1.62 & 0.37 & 1.07 & 0.15 & 1.09 & 0.17 & 1.65 & 0.06 & 2.59 & 0.69 & 0.25 \\
\hline 5.83 & 0.84 & 4.55 & 1.13 & 0.39 & 1.39 & 0.24 & 1.61 & 0.29 & 0.98 & 0.14 & 1.06 & 0.21 & 1.54 & WC & 1.80 & 0.46 & 0.18 \\
\hline 15.8 & 1.81 & 7.91 & 1.51 & 0.53 & 1.84 & 0.35 & 2.46 & 0.57 & 1.90 & 0.33 & 2.15 & 0.32 & 1.65 & 0.06 & 2.44 & 0.62 & 0.36 \\
\hline 11.7 & 1.35 & 7.09 & 1.65 & 0.47 & 2.19 & 0.39 & 2.23 & 0.52 & 1.67 & 0.28 & 1.71 & 0.28 & 1.99 & WC & 1.52 & 0.39 & 0.18 \\
\hline 5.37 & 0.75 & 3.74 & 1.02 & 0.34 & 1.15 & 0.20 & 1.20 & 0.27 & 0.72 & 0.16 & 0.81 & 0.13 & 1.19 & 0.04 & 6.21 & 0.48 & 0.27 \\
\hline 5.25 & 0.82 & 4.03 & 1.30 & 0.41 & 1.30 & 0.25 & 1.54 & 0.37 & 1.03 & 0.18 & 1.54 & 0.14 & 1.62 & WC & 2.32 & 0.35 & 0.27 \\
\hline 5.63 & 0.58 & 4.20 & 0.99 & 0.32 & 1.12 & 0.24 & 1.30 & 0.28 & 0.85 & 0.12 & 0.93 & 0.14 & 1.92 & WC & 2.72 & & \\
\hline 8.04 & 1.18 & 5.74 & 1.26 & 0.41 & 1.28 & 0.32 & 1.78 & 0.40 & 1.17 & 0.22 & 1.22 & 0.23 & 2.20 & 0.06 & 3.72 & 0.66 & 0.29 \\
\hline 6.72 & 0.96 & 4.79 & 1.16 & 0.37 & 1.59 & 0.25 & 1.49 & 0.32 & 1.03 & 0.18 & 1.02 & 0.13 & 1.76 & WC & 2.23 & 0.56 & 0.28 \\
\hline 5.46 & 0.56 & 3.35 & 0.97 & 0.30 & 1.18 & 0.22 & 1.28 & 0.23 & 0.86 & 0.13 & 0.97 & 0.16 & 1.92 & WC & 2.20 & & \\
\hline 7.13 & 1.09 & 5.10 & 1.31 & 0.37 & 1.57 & 0.30 & 1.71 & 0.36 & 1.16 & 0.20 & 1.22 & 0.22 & 2.74 & WC & 2.81 & 0.72 & 0.37 \\
\hline 9.44 & 1.29 & 6.12 & 1.47 & 0.43 & 1.95 & 0.32 & 1.90 & 0.43 & 1.25 & 0.20 & 1.35 & 0.23 & 2.37 & 0.07 & 3.47 & 0.76 & 0.40 \\
\hline 10.9 & 1.42 & 7.34 & 1.88 & 0.63 & 2.43 & 0.37 & 2.50 & 0.55 & 1.42 & 0.24 & 1.43 & 0.23 & 2.90 & 0.12 & 2.35 & 0.83 & 0.43 \\
\hline 7.41 & 0.81 & 4.65 & 1.84 & 0.49 & 1.82 & 0.27 & 1.57 & 0.33 & 1.01 & 0.16 & 1.06 & 0.15 & 2.43 & WC & 2.43 & 0.71 & 0.50 \\
\hline 8.28 & 1.08 & 5.41 & 1.55 & 0.41 & 1.95 & 0.33 & 1.68 & 0.35 & 1.21 & 0.18 & 1.27 & 0.23 & 2.32 & WC & 3.19 & 0.67 & 0.37 \\
\hline 6.16 & 0.81 & 4.72 & 1.35 & 0.51 & 1.42 & 0.19 & 1.53 & 0.29 & 0.92 & 0.18 & 1.16 & 0.13 & 2.22 & 0.06 & 2.46 & 0.52 & \\
\hline 3.80 & 0.33 & 1.74 & 0.56 & 0.28 & 0.80 & 0.15 & 0.99 & 0.20 & 0.58 & 0.10 & 0.61 & 0.08 & 1.28 & 0.09 & 4.80 & 0.27 & 0.21 \\
\hline 4.23 & 0.80 & 4.54 & 1.63 & 0.63 & 2.31 & 0.41 & 2.94 & 0.63 & 1.90 & 0.28 & 2.03 & 0.38 & 1.14 & WC & 3.16 & 0.07 & 0.07 \\
\hline 2.45 & 0.39 & 1.93 & 0.51 & 0.18 & 0.68 & 0.14 & 1.07 & 0.23 & 0.62 & 0.11 & 0.72 & 0.15 & 0.82 & 0.03 & 0.86 & 0.13 & 0.20 \\
\hline 1.43 & 0.21 & 1.11 & 0.40 & 0.15 & 0.52 & 0.09 & 0.69 & 0.15 & 0.41 & 0.09 & 0.56 & 0.07 & 0.75 & 0.05 & 0.68 & 0.12 & 0.26 \\
\hline
\end{tabular}




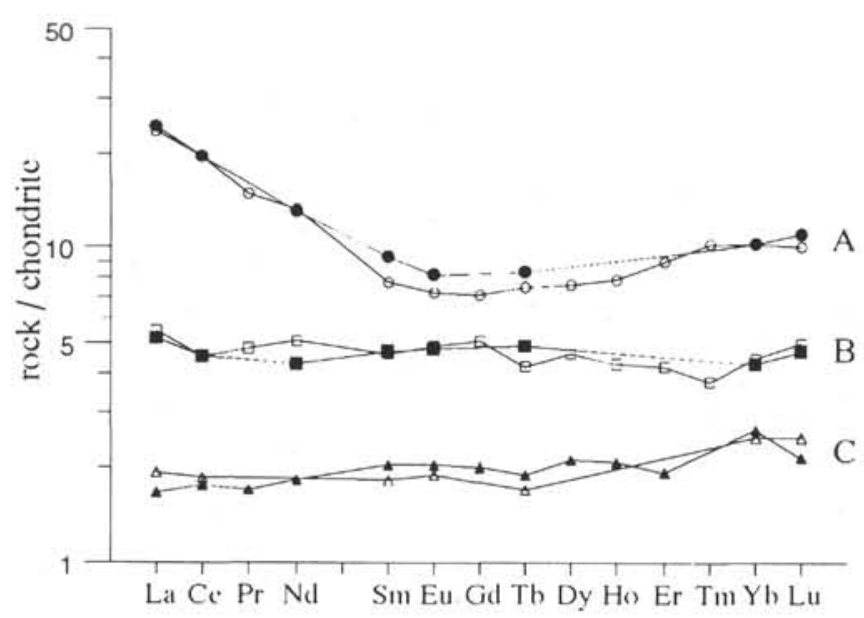

Figure 5. Chondrite-normalized REE patterns (normalizing values from Boynton, 1984) showing comparison between INAA (filled symbols) and ICP-MS (open symbols) data for three Site 786 samples. $A=786 \mathrm{~B}-21 \mathrm{R}-1$, $20-28 \mathrm{~cm} ; \mathrm{B}=786 \mathrm{~B}-67 \mathrm{R}-1,56-59 \mathrm{~cm} ; \mathrm{C}=786 \mathrm{~A}-17 \mathrm{X}-\mathrm{CC}, 4-12 \mathrm{~cm}$.

slightly higher $\mathrm{Cr}$ contents (333-1004 ppm; average 755). They also have the lowest $\mathrm{Zr}(22-33 \mathrm{ppm})$ and $\mathrm{Th}(<0.22 \mathrm{ppm})$ contents of all the groups, although Y tends to be slightly higher than for the other boninite types, and $\mathrm{Sr}$ contents (100-150 ppm) lie between those of the ICB's and LCB's. They contain 3-13 modal percent phenocrysts of: bronzite $(0 \%-8 \%)$, augite $(0 \%-3 \%)$, olivine $(3 \%-5 \%)$, and traces of euhedral chromium-spinel.

\section{Andesite-Dacite-Rhyolite Sequence}

The Andesite-Dacite-Rhyolite sequence (ADR) is clearly distinguished from the more primitive boninite and bronzite andesite groups by their low $\mathrm{Cr}$ and $\mathrm{Ni}$ contents $(<100 \mathrm{ppm}$ and $<50 \mathrm{ppm}$ respectively). $\mathrm{Zr}$ contents increase between the groups as do $\mathrm{P}_{2} \mathrm{O}_{5}$, $\mathrm{Ba}, \mathrm{Y}$, and $\mathrm{Rb}$, although these latter elements show less distinct behavior because of alteration effects. Both $\mathrm{TiO}_{2}$ and $\mathrm{V}$ show a progressive decrease from andesite through dacite to rhyolite. The three members of the ADR series are described in detail below.

\section{Andesites}

Andesites (A) of ca. $41 \mathrm{Ma}$ age occur as flows (Cores 20R-21R and 37R-40R), breccias interbedded with ICBA's and dacites (Cores 7R-10R, 14R-17R and 48R-51R), and as a dike (Cores 64R-65R): Compared with the ICBA's, they form a group that has distinctively low $\mathrm{MgO}$ (1.7-5.7 wt\%), lower Mg\# (30.8-55.7), similar $\mathrm{SiO}_{2}(56.5-$ $62.4 \mathrm{wt} \%)$ and $\mathrm{CaO}(5.4-7.9 \mathrm{wt} \%)$, and distinctly higher $\mathrm{TiO}_{2}(0.24$ $0.45 \mathrm{wt} \%$; average 0.32 ). They are less primitive than the LCBA's, with low Ni contents (13-41 ppm) and low Cr contents (7.6-67 ppm; average 21 ). $\mathrm{Zr}$ generally increases from 40 to $60 \mathrm{ppm}$. Both $\mathrm{Sr}$ and $\mathrm{Ba}$ increase with increasing $\mathrm{SiO}_{2}(160-290 \mathrm{ppm}$ and $18-80 \mathrm{ppm}$, respectively), although $\mathrm{Rb}$ is very variable. Crystal contents range from 2 to 12 modal percent of orthopyroxene (trace amounts), augite $(0 \%-5 \%)$, and plagioclase $(4 \%-10 \%)$. Magnetite and rare quartz and/or amphibole are present in the groundmass.

\section{Dacites}

Dacites (D) of ca. 41 Ma age occur as flows (Cores 17R-20R and 23R-27R and 34R-37R), breccias interbedded with andesites (Cores $7 \mathrm{R}-10 \mathrm{R}$ ), and as a dike (Cores 64R-65R). In addition to higher $\mathrm{SiO}_{2}$ (64.9-68.9 wt\%), they have distinctly low $\mathrm{MgO}(0.57-3.4 \mathrm{wt} \%), \mathrm{Mg} \#$ (16.5-49.3) and $\mathrm{CaO}(3.9-5.7 \mathrm{wt} \%)$, and similar $\mathrm{TiO}_{2}(0.29-0.34$; average $0.32 \mathrm{wt} \%$ ) compared to the andesite group. They are less primitive than the andesites, with lower $\mathrm{Ni}$ contents $(6.8-26 \mathrm{ppm}$; average 12), and lower $\mathrm{Cr}$ contents (6.8-26; average $19 \mathrm{ppm}) . \mathrm{Zr}$ and $\mathrm{Y}$ increase with $\mathrm{SiO}_{2}$ (35-71 ppm and 5.5-15 ppm, respectively). $\mathrm{Sr}$ decreases with increasing $\mathrm{SiO}_{2}(220-190 \mathrm{ppm})$, but $\mathrm{Rb}$ and $\mathrm{Ba}$ both increase (8-14 and 23-84 ppm, respectively). Crystal contents range from 3 to 20 modal percent of: orthopyroxene and augite (trace amounts), and plagioclase (4\%-20\%). Pigeonite, augite, magnetite, and rare quartz and/or amphibole are present in the groundmass.

\section{Rhyolites}

Rhyolite (R) of ca. 41 Ma age occurs as hyaloclastite bearing flows (Cores 32R-34R, Sections 21R-121R-2), massive flows interbedded with ICBA, D, and LCB (63R-68R), and as a dike (Sections 61R-4 61R-6). In addition to higher $\mathrm{SiO}_{2}$ (70.9-76.3 wt\%), they have distinctively lower $\mathrm{MgO}(0.01-1.5 \mathrm{wt} \%), \mathrm{Mg \#}(0.9-39.6)$ and $\mathrm{CaO}$ $(0.45-3.5 \mathrm{wt} \%)$, and low $\mathrm{TiO}_{2}(0.23-0.27 \mathrm{wt} \%$; average 0.24$)$ compared to the dacites. They are the most evolved lithologies in Hole $786 \mathrm{~B}$ with the lowest $\mathrm{Ni}$ contents $(1.1-11 \mathrm{ppm})$, and lowest $\mathrm{Cr}$ contents (3-33 ppm; average 15). Sr decreases rapidly with increasing $\mathrm{SiO}_{2}$, although $\mathrm{Rb}$ and $\mathrm{Ba}$ form a somewhat scattered, but greatly increasing, trend. $\mathrm{Zr}$ remains virtually constant, and $\mathrm{Y}$ shows slightly decreasing abundances with increasing $\mathrm{SiO}_{2}$ due to alteration in Unit 29 . The rhyolites also have distinctly low $\mathrm{V}$ contents $(<50 \mathrm{ppm})$. Crystal contents are low with 1-2 modal percent of plagioclase, and only trace amounts of hypersthene and augite.

\section{ALTERATION EFFECTS}

The igneous lithologies encountered at Site 786 exhibit a wide variety in the degree of alteration (up to greenschist-facies albitechlorite-epidote-quartz assemblages) largely resulting from the effects of hydrothermal alteration during cooling of the edifice/basement complex and during intrusion of the late dikes and sills. Despite this, Arculus et al. (this volume) conclude that the major elements used to classify the groups are robust with respect to this alteration, in the sense that alteration-induced variations are small relative to primary magmatic variations. However, it is apparent from the diagrams of Figure 6 that these alteration processes have disturbed the distribution of certain trace-elements. It is important to understand and take account of the effects of alteration on the trace-element composition of samples before reading any petrogenetic significance into the observed variations. A number of distinct styles of trace-element behavior can be recognized.

As noted earlier, about 12 samples show a clear anomalous enrichment of $\mathrm{P}_{2} \mathrm{O}_{5}$ and $\mathrm{Y}$ (Fig. 6). These samples are not restricted to any particular chemical group although they tend to be found only within the breccia horizons. This enrichment reaches its most extreme development in the ICBA Sample 48R-1, 48-53 cm, which contains $1.59 \mathrm{wt} \% \mathrm{P}_{2} \mathrm{O}_{5}$ and 95 ppm Y (omitted from diagrams in Fig. 6). A similar style of enrichment has also been documented from lavas at Site 793 in the Izu-Bonin forearc basin drilled during ODP Leg 126 (Taylor et al., in press). Taylor et al. attributed the anomalous behavior of P, Y, and rare earth elements (REE) relative to high field strength elements (HFSE) to post-eruption fluid-rock interaction and traceelement release associated with the breakdown of glass to smectite within the basement units.

By comparing the compositions of two samples from the same lithological subunit, one with "normal" magmatic values for $\mathrm{P}_{2} \mathrm{O}_{5}$ and $\mathrm{Y}$ and one with "elevated" $\mathrm{P}_{2} \mathrm{O}_{5}$ and $\mathrm{Y}$, we can ascertain which other trace elements are affected by this enrichment process. Figure 7 shows this comparison for an andesite (21R-1, 20-28 cm, and 19R-1, 91-94 $\mathrm{cm}$ ) and an intermediate-Ca boninite (51R-1, 5-55 cm, and 51R-1, $75-79 \mathrm{~cm}$ ) composition. The pattern of trace-element enrichment is remarkably similar in the two examples illustrated, despite their belonging to different chemical groups. In both cases, the elevated $\mathrm{P}$ and $\mathrm{Y}$ contents are accompanied by higher REE abundances, but there 
Table 4. INAA trace element data on selected samples.

\begin{tabular}{|c|c|c|c|c|c|c|c|c|c|c|c|c|c|c|c|c|c|c|}
\hline Hole & Core & $\begin{array}{l}\text { Interval } \\
(\mathrm{cm})\end{array}$ & $\begin{array}{l}\text { Chemical } \\
\text { group }\end{array}$ & Sc & $\mathrm{Co}$ & $\mathrm{Cs}$ & La & $\mathrm{Ce}$ & $\mathrm{Nd}$ & $\mathrm{Sm}$ & Eu & TbY & $\mathrm{Yb}$ & $\mathrm{Lu}$ & $\mathrm{Hf}$ & $\mathrm{Ta}$ & Th & $\mathrm{U}$ \\
\hline $786 \mathrm{~A}$ & $12 X-1$ & $133-138$ & misc. & 30.4 & 26 & & 0.9 & 3.7 & 2.1 & 0.59 & 0.29 & 0.14 & 0.61 & 0.09 & 1.16 & 0.04 & 0.39 & 0.23 \\
\hline $786 \mathrm{~A}$ & $16 \mathrm{X}-\mathrm{CC}$ & $24-29$ & misc. & 29.4 & 40 & & 1.1 & 2.4 & 2.2 & 0.65 & 0.25 & 0.15 & 0.77 & 0.12 & 0.86 & 0.04 & & 0.10 \\
\hline $786 \mathrm{~A}$ & $17 \mathrm{X}-\mathrm{CC}$ & $4-12$ & misc. & 24.8 & 41 & 0.25 & 0.6 & 1.5 & & 0.36 & 0.14 & 0.08 & 0.53 & 0.08 & 0.68 & 0.02 & & 0.31 \\
\hline $786 \mathrm{~B}$ & $05 \mathrm{R}-2$ & $69-71$ & HCB & 33.6 & 40 & 0.31 & 2.0 & 4.1 & 3.5 & 1.08 & 0.42 & 0.27 & 1.07 & 0.17 & 0.87 & 0.02 & & 0.31 \\
\hline $786 \mathrm{~B}$ & $21 R-2$ & $85-94$ & $\mathrm{HCB}$ & 28.7 & 45 & & 1.5 & 3.0 & 2.2 & 0.60 & 0.23 & 0.15 & 0.68 & 0.11 & 0.75 & 0.03 & 0.38 & 0.20 \\
\hline $786 \mathrm{~B}$ & $40 \mathrm{R}-2$ & $83-90$ & HCB & 29.8 & 45 & 0.41 & 1.4 & 3.3 & 2.7 & 0.80 & 0.33 & 0.21 & 0.86 & 0.12 & 0.67 & 0.02 & 0.30 & 0.14 \\
\hline $786 \mathrm{~B}$ & $11 R-1$ & $121-123$ & ICB & 30.9 & 36 & 0.82 & 1.7 & 3.0 & 2.2 & 0.75 & 0.29 & 0.22 & 1.01 & 0.15 & 0.74 & 0.03 & & 0.32 \\
\hline $786 \mathrm{~B}$ & $51 R-1$ & $75-79$ & ICB & 28.3 & 37 & & 2.3 & 5.3 & 3.5 & 1.04 & 0.36 & 0.23 & 0.87 & 0.14 & 0.97 & 0.04 & 0.47 & 0.17 \\
\hline $786 \mathrm{~B}$ & $67 R-1$ & $56-59$ & ICB & 33.6 & 39 & & 1.6 & 3.7 & 2.6 & 0.92 & 0.35 & 0.23 & 0.90 & 0.15 & 0.98 & 0.04 & 0.36 & 0.18 \\
\hline $786 \mathrm{~B}$ & $57 \mathrm{R}-4$ & $7-13$ & LCB & 30.9 & 42 & & 1.6 & 4.0 & 3.0 & 0.79 & 0.29 & 0.17 & 0.73 & 0.11 & 0.98 & 0.04 & 0.34 & 0.15 \\
\hline $786 \mathrm{~B}$ & $62 R-3$ & $40-42$ & LCB & 25.3 & 19 & & 2.7 & 5.2 & 3.9 & 1.13 & 0.37 & 0.22 & 0.96 & 0.15 & 1.29 & 0.04 & 0.43 & 0.27 \\
\hline $786 \mathrm{~B}$ & 0IR-1 & $75-79$ & ICBA & 25.6 & 29 & 0.20 & 2.0 & 4.6 & 2.9 & 0.84 & 0.30 & 0.18 & 0.72 & 0.11 & 1.22 & 0.05 & & 0.22 \\
\hline $786 \mathrm{~B}$ & $30 \mathrm{R}-1$ & $30-32$ & ICBA & 31.0 & 31 & 0.29 & 1.6 & 3.1 & & 0.66 & 0.24 & 0.16 & 0.57 & 0.09 & 0.86 & 0.04 & & 0.19 \\
\hline $786 \mathrm{~B}$ & $70 R-1$ & $92-97$ & LCBA & 27.6 & 32 & & 1.6 & 3.4 & 2.4 & 0.75 & 0.34 & 0.18 & 0.75 & 0.12 & 1.00 & 0.04 & 0.30 & 0.28 \\
\hline $786 \mathrm{~B}$ & 08R-1 & $50-55$ & AND & 25.3 & 24 & & 2.6 & 5.8 & 5.8 & 1.74 & 0.60 & 0.36 & 1.16 & 0.19 & 1.42 & 0.05 & 0.48 & 0.16 \\
\hline $786 \mathrm{~B}$ & $19 \mathrm{R}-1$ & $91-94$ & AND & 29.0 & 45 & & 1.9 & 3.7 & 3.1 & 0.83 & 0.26 & 0.18 & 0.79 & 0.13 & 0.90 & 0.03 & 0.20 & 0.30 \\
\hline $786 \mathrm{~B}$ & $21 R-1$ & $20-28$ & AND & 22.6 & 11 & & 7.6 & 15.7 & 7.8 & 1.82 & 0.60 & 0.39 & 2.13 & 0.35 & 1.59 & 0.07 & 0.55 & 0.42 \\
\hline $786 \mathrm{~B}$ & $49 \mathrm{R}-4$ & $32-37$ & AND & 25.0 & 22 & 0.32 & 2.6 & 4.7 & 3.9 & 1.15 & 0.38 & 0.24 & 1.07 & 0.16 & 1.20 & 0.05 & 0.36 & 0.32 \\
\hline $786 \mathrm{~B}$ & $35 \mathrm{R}-1$ & $75-78$ & DAC & 20.4 & 14 & & 2.9 & 6.2 & 4.5 & 1.24 & 3.41 & 0.23 & 1.06 & 0.16 & 1.57 & 0.06 & 0.50 & 0.23 \\
\hline $786 \mathrm{~B}$ & $21 \mathrm{R}-2$ & $28-32$ & RHY & 11.4 & 8 & 0.82 & 4.0 & 8.9 & 5.9 & 1.65 & 0.47 & 0.31 & 1.38 & 0.21 & 2.19 & 0.08 & 0.75 & 0.48 \\
\hline $786 \mathrm{~B}$ & $66 \mathrm{R}-1$ & $26-33$ & RHY & 11.8 & 6 & 0.25 & 3.6 & 8.1 & 5.9 & 1.39 & 0.46 & 0.26 & 1.17 & 0.19 & 2.10 & 0.08 & 0.70 & 0.40 \\
\hline
\end{tabular}

is preferential enrichment of both the LREE and HREE over the middle REE. This leads to higher $\mathrm{La} / \mathrm{Sm}, \mathrm{La} / \mathrm{Yb}$, and lower $\mathrm{Sm} / \mathrm{Yb}$ ratios with alteration. Immobile elements during this alteration process include the HFSE $(\mathrm{Zr}, \mathrm{Hf}, \mathrm{Ti}, \mathrm{Nb})$ as well as $\mathrm{Sr}$ and $\mathrm{Pb}$. The large-ion-lithophile (LIL) elements (such as $\mathrm{K}$ and $\mathrm{Rb}$ ) show no consistent behavior.

Because $\mathrm{Zr}$ is one of the most immobile and easily analyzed elements, it can be used as an alteration-independent index of geochemical variation. The behavior of certain other trace-elements can then be assessed by plotting the element in question against $\mathrm{Zr}$. Figure 8 shows plots of $\mathrm{Sr}$, $\mathrm{Th}, \mathrm{Pb}$, and $\mathrm{Rb}$ against $\mathrm{Zr}$. The intermediate-Ca boninites and bronzite andesites and the late-dikes plot on a trend of increasing Sr with increasing $\mathrm{Zr}$ (Fig. 8A), consistent with fractional crystallization of mafic phases. The trend continues into the andesites, dacites, and rhyolites where the appearance of plagioclase as a significant crystallizing phase leads to decreasing Sr abundances. The rhyolites show a wide range of Sr contents $(50-0 \mathrm{ppm})$ for a restricted range of $\mathrm{Zr}$ contents $(70-0 \mathrm{ppm})$, but the low values are only found in the strongly altered subunit 29 in which $\mathrm{Sr}$ may have been mobile. The alteration of this unit is discussed in more detail below. Although the scatter within these trends exceeds analytical error, many of the samples are highly porphyritic (some plagioclase-phyric) which will enhance primary scatter. The effects of plagioclase accumulation are noticeable in some andesite and intermediate-Ca bronzite-andesite samples displaced to high $\mathrm{Sr}$ contents for a given $\mathrm{Zr}$ content. The low-Ca boninites and bronzite-andesites form a trend subparallel to the other boninite/bronzite-andesite groups but displaced to lower Sr. Although the low-Ca boninite units are more altered, the fact that the dispersion of analyses is parallel to the expected crystallization vector for olivine + orthopyroxene fractionation, suggests that the low $\mathrm{Sr}$ is a primary, rather than alteration-induced, feature. Figure 7 has already shown that $\mathrm{Sr}$ was not mobilized during the process that lead to the enrichments in $\mathrm{P}, \mathrm{Y}$, and REE. This behavior of $\mathrm{Sr}$ is in contrast with its theoretically expected highly mobile nature in aqueous fluids. Taylor et al. (in press) encountered the same features in boninite samples from ODP Leg 126, and they argued that, because $\mathrm{Sr}$ is located mostly within plagioclase, it may be less mobile than many of the normally more immobile incompatible elements that reside within the much more reactive boninite glass. Thus, with the exception of the rhyolite unit at the base of Hole 786B, the primary magmatic variations of Sr appear to have remained relatively robust to the effects of alteration. All of the Site 786B samples show a significant positive correlation between $\mathrm{Th}$ and $\mathrm{Zr}$ (Fig. $8 \mathrm{~B})$. The magnitude of the scatter is consistent with pyroxene accumulation and analytical error suggesting that $\mathrm{Th}$, like $\mathrm{Zr}$, behaved as an immobile element. The $\mathrm{Pb}$ data on Figure $8 \mathrm{C}$ show a larger scatter, well in excess of analytical error. For example, $\mathrm{Pb}$ contents in the low $\mathrm{Ca}$ boninites and bronzite-andesites vary between 1.5 and 4 ppm over a range in $\mathrm{Zr}$ of just $30-45 \mathrm{ppm}$, which suggests that $\mathrm{Pb}$ has been mobilized by alteration.

The mobility of LIL elements during hydrothermal alteration processes is well documented, and the more scattered $\mathrm{Rb}$ values on Figure 8 and $\mathrm{Ba}$ values on Figure 6 reflect this. If the variation within a particular chemical group is attributed principally to the action of crystal fractionation or accumulation, then the observed range in $\mathrm{Rb}$ and $\mathrm{K}_{2} \mathrm{O}$ contents in most groups far exceeds that predicted from the variation in a less mobile incompatible element such as $\mathrm{Zr}$. For example, within the $\mathrm{HCB}$ group, $\mathrm{K}_{2} \mathrm{O}$ varies by a factor of 7 (0.19-1.3 $\mathrm{wt} \%)$ and $\mathrm{Rb}$ by a factor of 5 (3-15 ppm) whereas $\mathrm{Zr}$ is enriched by a factor of only 1.5 (23-34 ppm). However, the mobility of the alkali elements is best displayed within the rhyolite units which, despite a relatively restricted range in $\mathrm{Zr}$ contents ( $70-82 \mathrm{ppm})$, show very large enrichments in $\mathrm{K}_{2} \mathrm{O}, \mathrm{Rb}$, and $\mathrm{Ba}(1.0-5.0 \mathrm{wt} \%, 15-51 \mathrm{ppm}$, $52-756 \mathrm{ppm}$, respectively). The most intensely altered of the rhyolite units is subunit 29 , from near the base of Hole $786 \mathrm{~B}$, which contains greenschist mineral assemblages. It is rhyolites from this unit that show the greatest $\mathrm{K}, \mathrm{Rb}$, and $\mathrm{Ba}$ enrichments. Figure 9 shows the average composition for this unit, normalized against an average of samples taken from the less intensely altered rhyolite subunits. This figure illustrates that the enrichment in $\mathrm{K}, \mathrm{Rb}$, and $\mathrm{Ba}$ is accompanied by a loss of $\mathrm{Ca}, \mathrm{Na}, \mathrm{P}, \mathrm{Sr}$, and $\mathrm{Y}$.

\section{SHALLOW-LEVEL PROCESSES}

From the major and trace-element variations illustrated in Figures 3 and 6 , it can be seen that the operation of fractional crystallization processes alone is unable to account for all of the compositional variability observed. However, the chemical groups can be grouped together into a number of cogenetic series, with the samples in each series related to a distinct parental magma through the action of crystal fractionation or accumulation. The Site 786 chemical groups are divided into four series and which are discussed in turn:

1. LCB series (low-Ca boninites and bronzite-andesites).

2. ICB series (Intermediate-Ca boninite and bronzite-andesites of the 41 Ma edifice).

3. ADR series (andesites, dacites, and rhyolites).

4. Late dike series (high-Ca boninites and intermediate-Ca boninites). 

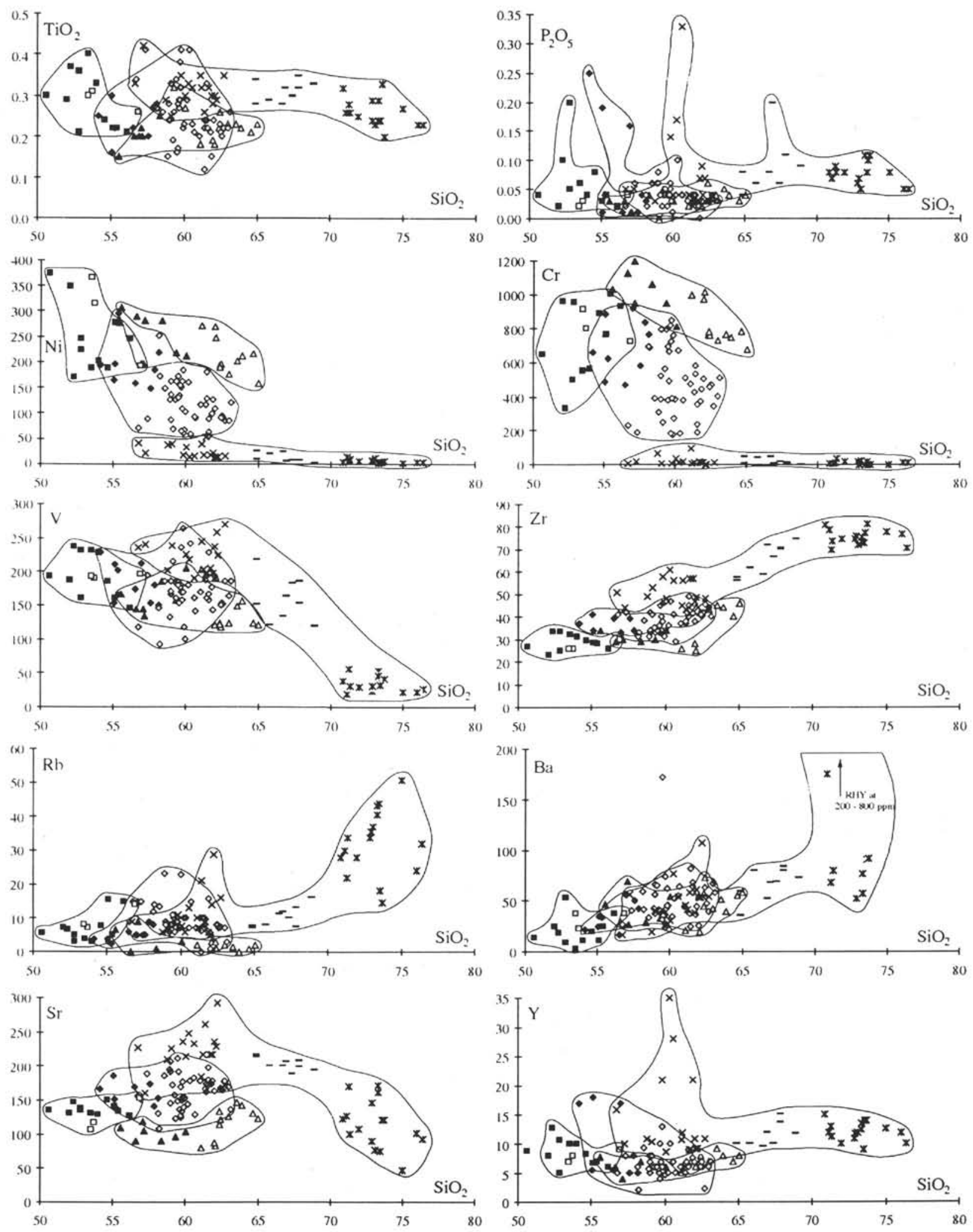

$\triangle \mathrm{LCB} \triangle \mathrm{LCBA}-\mathrm{KCB}$ O $\mathrm{ICBA} \times \mathrm{AND}-\mathrm{DAC} \times \mathrm{RHY}-\mathrm{HCB}$ 口 $\mathrm{KCB}$

Figure 6. Trace element vs. $\mathrm{SiO}_{2}$ variation diagrams. Samples are grouped together into four series (see text): LCB series (triangles), ICB series (diamonds), ADR series (crosses, dashes), late dikes (squares). Note anomalous alteration-related enrichment of $\mathrm{P}$ and $\mathrm{Y}$, and wide variation of Rb, $\mathrm{Ba}, \mathrm{Sr}$ contents in rhyolites. 


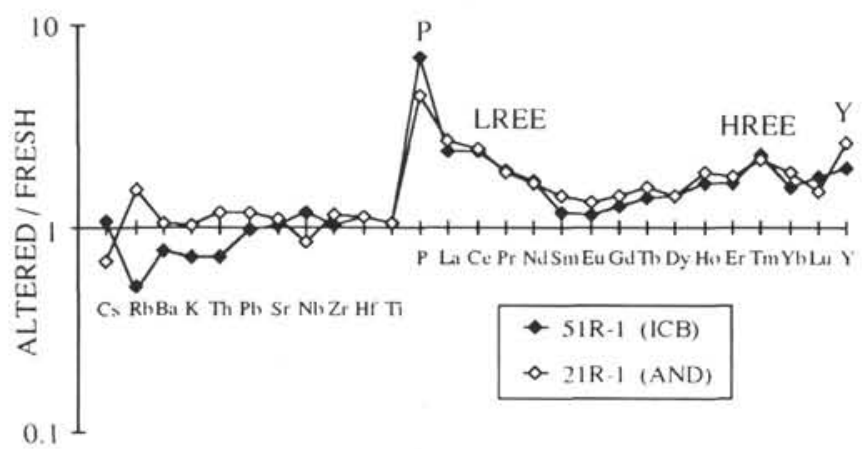

Figure 7. Trace element concentrations of "altered" sample normalized to "fresh" sample. Enrichment of $\mathrm{P}$ and $\mathrm{Y}$ is accompanied by enrichment of LREE and HREE during alteration, with $\mathrm{HFS}$ elements $(\mathrm{Zr}, \mathrm{Hf}, \mathrm{Ti}, \mathrm{Nb}, \mathrm{Ta})$ and $\mathrm{Sr}$ remaining immobile. Solid diamonds $=$ intermediate-Ca boninite $(51 \mathrm{R}-1$, 51-55 cm vs. $51 \mathrm{R}-1,75-79 \mathrm{~cm})$, open diamonds = andesite $(21 \mathrm{R}-1,20-28$ cm, vs. 19R-1, 91-94 cm).

\section{LCB Series}

Low-Ca boninites have a close spatial association with low-Ca bronzite-andesites and are found locally interspersed with low-Ca bronzite-andesite pillow lavas. On the $\mathrm{MgO}$ vs. $\mathrm{SiO}_{2}$ diagram (Fig.
3), the low-Ca bronzite-andesites appear to lie on an olivine- and orthopyroxene-controlled liquid line of descent from the low-Ca boninites, which would be in keeping with the olivine-orthopyroxene dominated petrography of these two groups. However, Arculus et al. (this volume) found that these two groups are not related by simple fractionation vectors on Pearce-type plots of $(\mathrm{Mg}+\mathrm{Fe}) /(\mathrm{Ti}$ or $\mathrm{K})$ vs. $\mathrm{Si} /(\mathrm{Ti}$ or K) (see Pearce, 1968); instead they form two subparallel trends. In detail, the low-Ca bronzite-andesites are found in two lithological subunits, 27 and 33 (Fig. 2). The Unit 27 samples have similar $\mathrm{Cr}(980-1020 \mathrm{ppm})$ and $\mathrm{Zr}(25-28 \mathrm{ppm})$ to the low-Ca boninites (which are only found in Unit 27), but are displaced to higher $\mathrm{SiO}_{2}(61-62 \mathrm{wt} \%)$. However, the bronzite-andesites of unit 27 might actually be low-Ca boninites that have experienced silicification (60R-3, 102-108 cm, contains small quartz veins) as their elevated $\mathrm{SiO}_{2}$ content is the only major compositional feature that distinguishes them from low-Ca boninites. Samples from Unit 33, on the other hand, are more evolved than those from the low-Ca boninite group, having higher $\mathrm{SiO}_{2}(63-65 \mathrm{wt} \%)$ and $\mathrm{Zr}(40-46 \mathrm{ppm})$ and lower $\mathrm{Cr}$ (670-790 ppm), cf. low-Ca boninites (55- 60 wt\%, 29-41 ppm, 810-1200 ppm, respectively). However, they are also characterized by low $\mathrm{K}_{2} \mathrm{O}$ contents $(<0.3 \mathrm{wt} \%)$ and, since the majority of the low-Ca bronzite-andesites show strong alteration, they may have suffered variable potassium loss.

A further complication is the crystal-rich nature of the low-Ca bronzite andesites which contain $20 \%-30 \%$ phenocrysts (dominantly orthopyroxene \pm olivine \pm chromium-spinel). Thus geochemical
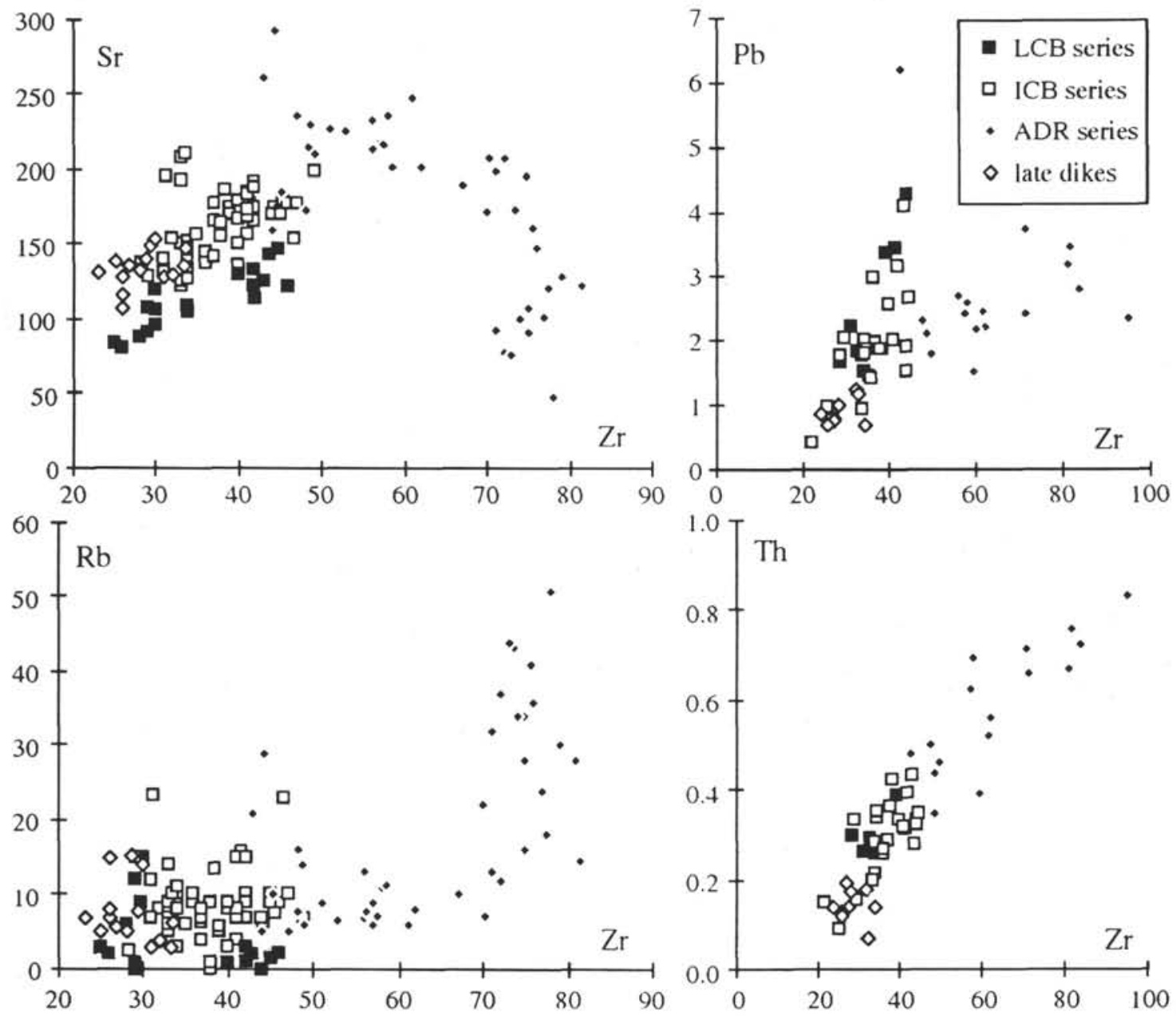

Figure 8. Trace element co-variation diagrams to highlight the effects of alteration on $\mathrm{Sr}, \mathrm{Rb}, \mathrm{Th}, \mathrm{Pb}$ relative to $\mathrm{Zr}$ (immobile). 


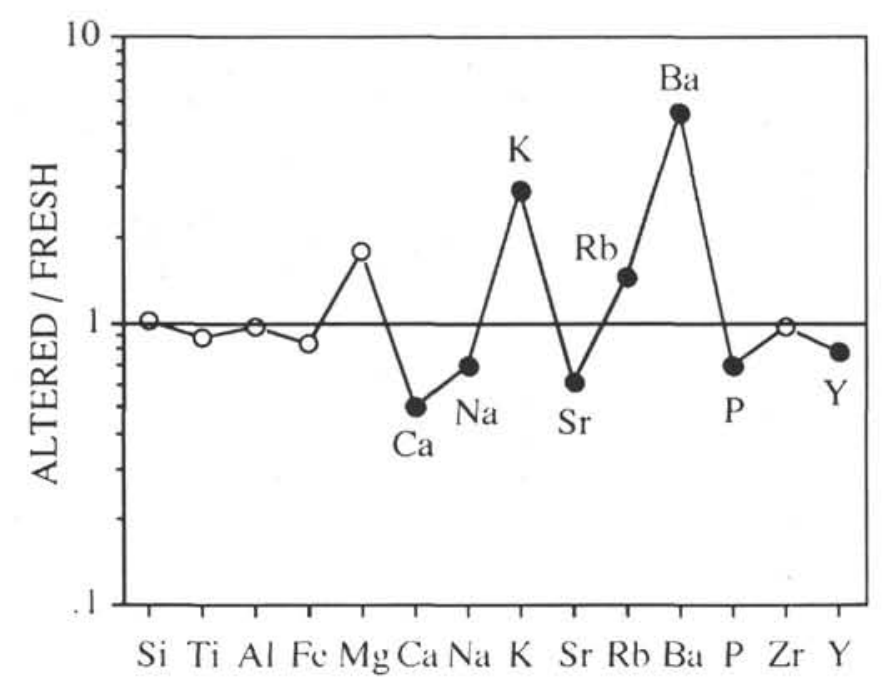

Figure 9. Average altered rhyolite (unit 29) composition normalized to average composition of less altered rhyolite (units 16, 20, 28). Alteration of unit 29 involved marked addition of $\mathrm{K}, \mathrm{Rb}, \mathrm{Ba}$ and loss of $\mathrm{Ca}, \mathrm{Na}, \mathrm{Sr}, \mathrm{P}, \mathrm{Y}$.
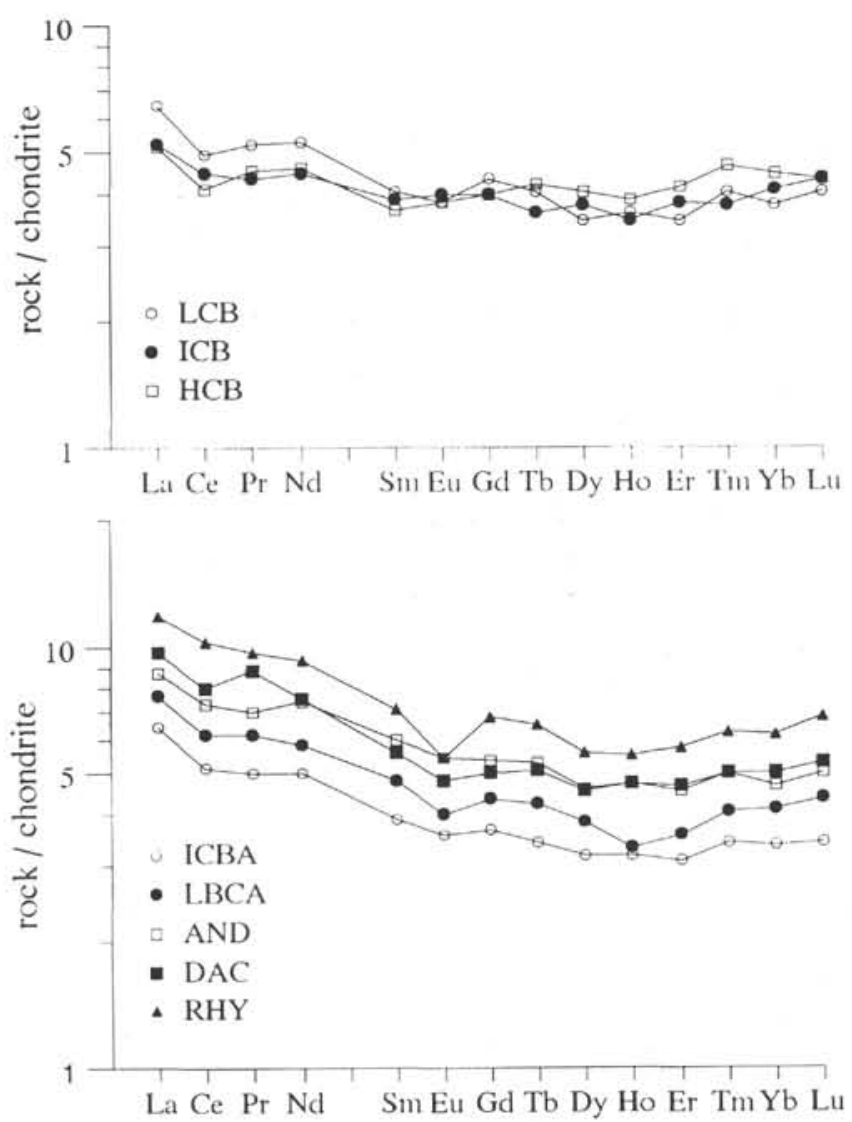

Figure 10. Chondrite-normalized REE patterns for averages of the eight lithologic groups from Site 786. Note the general similarities in patterns from the Eocene groups with shallow dish-shaped profiles caused by light-REE enrichment of otherwise heavy-REE and middle-REE element depleted abundances. This is not the case, however, for the late-dike series. Normalizing values are taken from Boynton (1984). analyses of these samples are unlikely to represent liquid compositions since their highly phyric nature effectively acts to dilute the content of incompatible trace elements and to elevate the contents of elements compatible with the cumulate phase(s). The low-Ca bronzite-andesites have similar HREE contents to the low-Ca boninites but with higher LREE contents (Ce/Yb: LCB 4.5-5.5, LCBA 5.5-7.0). On most trace-element variation diagrams (Figs. 6 and 8) both groups tend to form a coherent group. Furthermore, ratios of immobile incompatible elements such as $\mathrm{Th} / \mathrm{Zr}$ that are unaffected by crystallization, are the same in both groups. The fact that the $\mathrm{Sr} / \mathrm{Zr}$ ratio is also constant indicates that $\mathrm{Sr}$ is behaving incompatibly, and therefore that there is no significant role for plagioclase in the crystallizing assemblage. This is consistent with the general absence of plagioclase phenocrysts in the low-Ca boninites and bronzite-andesites. However, a negative Eu anomaly is present in some LCB samples (Fig. 10) which would be at odds with the petrographical evidence for fractionation of mafic phases only. This may be explained either by experimental error or, more probably, by the differential mobility of divalent and trivalent REE's during alteration. Thus the trace element data further support major element and isotopic evidence (Pearce et al., this volume) that the low-Ca boninites and bronzite-andesites form a cogenetic series linked by a complex combination of fractionation and accumulation dominated by orthopyroxene, olivine, and chromium-spinel.

\section{ICB Series}

The intermediate-Ca boninites and bronzite-andesites of the Eocene-age edifice form the dominant lithologies of Hole 786B. Samples from both groups are invariably crystal rich, with the boninites having $15 \%-25 \%$ phenocrysts and the bronzite-andesite having at least $15 \%$, but in general $20 \%-35 \%$, of phenocrysts. Phenocrysts in both groups include orthopyroxene $(2 \%-20 \%)$, clinopyroxene $(3 \%-10 \%)$, plagioclase $(0 \%-15 \%)$, olivine $(0 \%-5 \%)$, plus traces of chromium spinel. Arculus et al. (this volume) used Pearce-type major element diagrams to investigate the relationships within and between these two groups. They show that the trend of the intermediate- $\mathrm{Ca}$ boninite data leading to the field of intermediate-Ca bronzite-andesite analyses is controlled largely by crystallization and/or accumulation of olivine and pyroxene, whereas the variation within the bronzite-andesite samples is the result of crystallization/accumulation of plagioclase and pyroxene. Plagioclase involvement in the bronzite-andesite is reflected in the wide variation in $\mathrm{Sr} / \mathrm{Zr}$ ratio (3 to 6). Four samples, all from subunit 13 , are noticeably displaced to high $\mathrm{Sr}$ and low $\mathrm{Zr}$ on Figure 8A consistent with petrographic evidence for plagioclase accumulation. The bronzite-andesites show a wide compositional range for many trace-elements (e.g., Fig. 6). In the case of HFS elements such as $\mathrm{Zr}$, this encompasses the range displayed by the intermediate-Ca boninites; for example, the boninites contain 28-41 ppm $\mathrm{Zr}$ (average $35 \mathrm{ppm}$ ) and the bronziteandesites contain 29-49 ppm $\mathrm{Zr}$ (average $40 \mathrm{ppm}$ ). Because the bronzite-andesites are more evolved, they might be expected to have higher $\mathrm{Zr}$ contents. This can be illustrated further with the REE data (Fig. 10). The intermediate-Ca bronzite-andesites are more LREE enriched than the boninites (Ce/Yb: ICBA 4-8, ICB 3-5) but tend to have lower $\mathrm{Yb}$ contents $(0.5-0.8 \mathrm{ppm}$ vs. $0.7-1.1 \mathrm{ppm})$. Thus the apparent dilution of incompatible element abundances in the porphyritic bronzite andesites emphasizes the importance of crystal accumulation processes in their evolution. From petrographic observations and mineral composition data, van der Laan et al. (this volume) suggest that a range of melt compositions had been involved in the evolution of the ICB group and that the presence of a magma chamber was required to allow repeated replenishment by more primitive magmas during the crystallization process. The wide variability in the trace element abundances of the ICB series support their model. 


\section{ADR Series}

The andesite, dacite, and rhyolite groups lie on a pyroxene and plagioclase control line on the $\mathrm{MgO}-\mathrm{SiO}_{2}$ diagram (Fig. 3), and the trace-element variations observed between these groups are consistent with such a fractionation scheme. $\mathrm{Zr}$ contents show an increase between the groups from andesites $(40-60 \mathrm{ppm})$ through dacites $(55-75 \mathrm{ppm})$ to rhyolites $(70-80 \mathrm{ppm})$. The involvement of plagioclase in the crystallizing mineral assemblage is confirmed by the progressive decrease in $\mathrm{Sr}$ contents from andesite to dacite to rhyolite, and by the presence of pronounced negative Eu-anomalies in the dacite and rhyolite units (Fig. 10). The andesites and dacites generally contain abundant ( $3 \%-20 \%)$ plagioclase phenocrysts, and several of the less evolved andesite samples (e.g., 37R-3, 45-49 cm, and $38 \mathrm{R}-1,76-83 \mathrm{~cm})$ have high $\mathrm{Sr}$ contents $(>250 \mathrm{ppm})$ at low $\mathrm{Zr}$ contents $(<45 \mathrm{ppm})$ as a result of plagioclase accumulation (see Fig. $8 \mathrm{~A})$. Magnetite phenocrysts are found in all three groups, and the effects of magnetite fractionation can be seen in the progressive decrease in V content from andesite (190-270 ppm) through dacite $(120-220 \mathrm{ppm})$ to rhyolite $(<50 \mathrm{ppm})$ and in the decrease in $\mathrm{TiO}_{2}$ contents between the dacite and rhyolite groups (Fig. 6). The similarity of chondrite-normalized REE patterns between these three evolved groups also supports a simple cogenetic relationship (Fig. 10). There is a large variation in REE abundances within each group although this is primarily an alteration feature. The significantly greater HREE abundances for the rhyolite group, compared with the andesite and dacite groups, is consistent with the rhyolite group being the most evolved of all the lithologic groups.

The differentiated nature of the ADR series is illustrated by their higher HREE abundances relative to the bronzite-andesite or boninite groups. Although the andesites potentially could have been derived from a high-Ca boninite precursor via fractional crystallization of an olivine + pyroxene assemblage (see Fig. 3), several factors preclude such a relationship; (1) the age difference between the groups: the ADR sequence forms part of the $41 \mathrm{Ma}$ Eocene edifice whereas the high- $\mathrm{Ca}$ boninites occur exclusively as late-stage $35 \mathrm{Ma}$ intrusives, (2) the significant trace-element (e.g., $\mathrm{Th} / \mathrm{Zr}, \mathrm{Nb} / \mathrm{Zr}$ ) and isotopic differences (Pearce et al., this volume) between the ADR series and the high-Ca boninites. Instead, it is more likely that they are related to the intermediate-Ca bronzite andesites with which they share many trace-element and isotopic characteristics, and that the $\mathrm{SiO}_{2}$-poor andesite samples have undergone crystal accumulation. The major element Pearce-type plots used in Arculus et al. (this volume) illustrate the plausibility of a simple crystal fractionation relationship between the intermediate-Ca bronzite-andesites and the andesites. van der Laan et al. (this volume) envisage a scheme where the erupted andesites, dacites, and rhyolites represent filter-pressed extracts from the intermediate-Ca bronzite-andesites.

\section{Late Dike Series}

The younger igneous episode(s) produced only primitive boninitic magmas of high-Ca and intermediate-Ca affinities. These form a geochemically distinct group and, notwithstanding the age differences, do not appear to be cogenetic with any of the chemical groups of the main 41 Ma edifice-building volcanism. Pearce et al. (this volume) emphasize the distinctive isotopic composition of these late dikes, and important trace-element differences also exist between them and the Eocene-age magmatism. The late dikes have the lowest contents of $\mathrm{Th}$, and of $\mathrm{Nb}$ and $\mathrm{Ta}$, of all the Site 786 chemical groups and they are also characterized by the lowest $\mathrm{Ta} / \mathrm{Zr}$ ratios. The late dikes are displaced to higher $\mathrm{Y}$ and HREE contents relative to the other boninite groups and are characterized by low $\mathrm{Zr} / \mathrm{Y}(3-4)$ relative to the ICB and LCB series $(\mathrm{Zr} / \mathrm{Y} 4-8)$. In spite of the documented susceptibility of $\mathrm{Y}$ and the HREE to mobility during alteration in these rocks and the fact that one or two samples (e.g., 29R-1, 63-66 cm) have clearly experienced the "P-Y enrichment" style of alteration, most of the late-dike samples do not show the LREE enrichment which is also characteristic of the ICB series of the main edifice. This indicates not only that the parent magma(s) to the late dikes was compositionally distinct from the Eocene ICB parental magma, but that it is also probable that the elevated levels of $\mathrm{Y}$ and the HREE are a primary feature of the late-stage intrusive magmas. van der Laan et al. (this volume) note that the mineral assemblages of these boninites are unique to each dike or sill. This suggests that the magmas did not experience magma chamber processes but were produced as individual melt batches and directly emplaced as primitive intrusives.

\section{ORIGIN OF PARENTAL MAGMAS}

\section{Mantle Source Depletion and Degree of Melting}

From the previous section, it appears that all of the evolved chemical groups at Site 786 can be related by fractionation or accumulation of the observed mineral phases back to the three boninite groups, which represent distinct parental magmas. A similar conclusion is reached by Arculus et al. (this volume) from the major element data. The different contents of $\mathrm{MgO}, \mathrm{CaO}, \mathrm{TiO}_{2}, \mathrm{Ni}$, and $\mathrm{Cr}$ between the three boninite groups, at a given $\mathrm{SiO}_{2}$ content, cannot be explained by crystal fractionation processes and instead are a consequence of variations in the composition and/or degree of partial melting of their source(s). This view is also confirmed by the isotopic differences highlighted by Pearce et al. (this volume). The underlying petrogenetic causes that gave rise to the three distinct parent magmas in the Site 786 region of the Izu-Bonin forearc must now be addressed, i.e., whether they can be derived either by variable degrees of melting of a single mantle source or from several compositionally distinct source regions, and also what inferences can be made about the nature of the source mantle and the melting regime.

The nature and degree of depletion of the mantle source(s) involved in the boninite generation can be investigated by plotting a compatible element (e.g., $\mathrm{Cr}$ ) against an incompatible element (e.g., Ti or Y) that is not added to the source in the 'subduction' component. Figures $11 \mathrm{~A}$ and $\mathrm{B}$ show the variation of $\mathrm{Cr}$ with $\mathrm{TiO}_{2}$ and $\mathrm{Y}$ for the two boninite/bronzite-andesite series and the late boninite dikes. Vectors indicating the effects of $20 \%$ crystallization of olivine, orthopyroxene, clinopyroxene, plagioclase, and $0.1 \%$ crystallization of $\mathrm{Cr}$-spinel are also shown. The ICB series and late-dike group both show a typical fractionation trend of rapidly decreasing $\mathrm{Cr}$ as $\mathrm{Y}$ and $\mathrm{TiO}_{2}$ contents increase, consistent with crystallization of olivine + pyroxene \pm chromium-spinel. The trends are subparallel but with the late dikes displaced to higher $\mathrm{Y}$ and $\mathrm{TiO}_{2}$ relative to the ICB series. The effects of orthopyroxene-spinel \pm olivine accumulation in the crystal-rich low-Ca bronzite-andesites are clearly demonstrated in Figure 11, the LCB series forming a relatively shallow trend with only minor decreases in $\mathrm{Cr}$ with increasing $\mathrm{Y}$ and $\mathrm{TiO}_{2}$ contents. The low-Ca boninites have similar $\mathrm{TiO}_{2}$ contents to the lower- $\mathrm{TiO}_{2}$ group of late dikes but lower $\mathrm{TiO}_{2}$ than the more primitive ICB series magmas. The wide range in Y contents in the low-Ca boninites is partly an analytical artifact of the XRF technique at these low abundances: ICP-MS analyses of these samples give a narrow range of 7.0 to $7.4 \mathrm{ppm} \mathrm{Y} \mathrm{(cf.} \mathrm{4-8} \mathrm{ppm} \mathrm{Y} \mathrm{by} \mathrm{XRF)} \mathrm{which} \mathrm{is} \mathrm{similar} \mathrm{to} \mathrm{the} \mathrm{Y} \mathrm{content}$ of the late-dike parental magma.

The low $\mathrm{Y}$ and $\mathrm{TiO}_{2}$ contents of the Site 786 boninites $(<10$ $\mathrm{ppm}$ and $<0.4 \mathrm{wt} \%)$ relative to arc-related volcanics $(>10 \mathrm{ppm}$ and $>0.5 \mathrm{wt} \%$ ) and MORB (>20 ppm and $>0.9 \mathrm{wt} \%)$ are apparent from Figure 11. Because fractional crystallization vectors are subvertical on these projections, this difference must be attributed to a number of possible source related features: (1) greater extent of source depletion, (2) larger degrees of melting, and (3) presence of residual Y- and Ti-bearing phases during partial melting (Pearce, 1982). However, the very low $\mathrm{Ti} / \mathrm{Sc}(35-70)$ and $\mathrm{Ti} / \mathrm{V}$ (5-12) ratios of the Site 786 boninites relative to MORB $(\mathrm{Ti} / \mathrm{Sc}>$ $100 ; \mathrm{Ti} / \mathrm{V}>18$ ) argue against the presence of residual clinopyroxene, garnet and amphibole (the most likely Y- and 

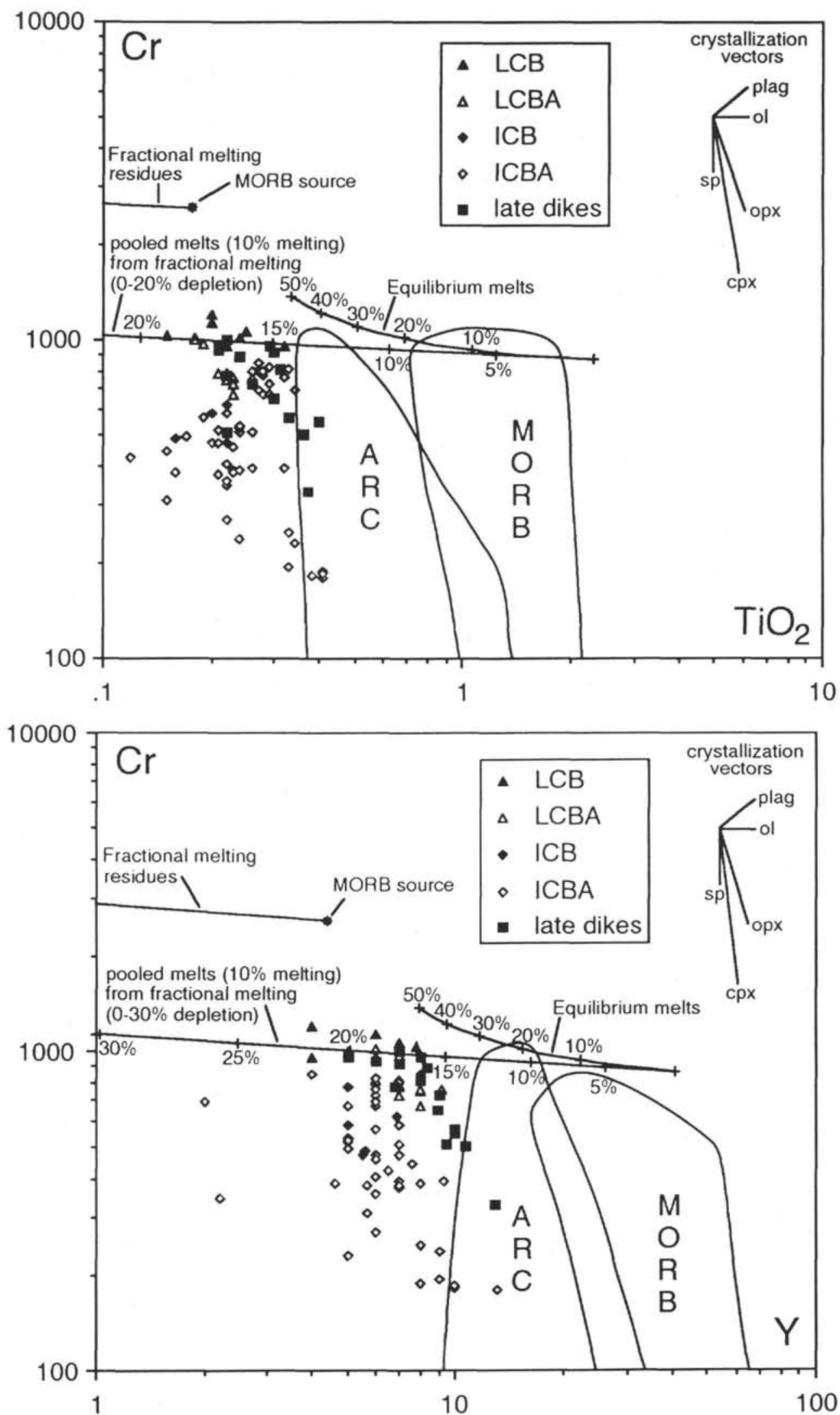

Figure 11. Melting trends in Cr, $\mathrm{Y}$ and Cr, Ti space, based on Pearce (1983). Trends for equilibirum and fractional melting of a MORB source are shown (see Parkinson et al., this volume, for details of melting models). Note the extremely low abundances of $\mathrm{Ti}$ and $\mathrm{Y}$ in the Site 786 boninites and bronzite-andesites relative to MORB and typical island arc rocks (fields for MORB and arcs from Pearce, 1975, 1982). 
Ti-bearing mantle phases) during melting of a MORB source mantle to explain the low $\mathrm{Y}$ and $\mathrm{Ti}$ contents of the boninites because $\mathrm{Sc}$ and $\mathrm{V}$ are retained preferentially with respect to $\mathrm{Ti}$ by these minerals (Hickey and Frey, 1982). Moreover, the high overall degree of melting involved and the mineralogy of the forearc peridotites similarly indicate that no residual $\mathrm{Y}$ - and Ti-bearing phases were present.

Melting trends for a MORB mantle source $\left(\mathrm{Cr}=2600 \mathrm{ppm}, \mathrm{TiO}_{2}\right.$ $=0.18 \mathrm{wt} \%, \mathrm{Y}=4 \mathrm{ppm}$ ) are superimposed on this figure using the equations, distribution coefficients, and parameters summarized in Parkinson et al. (this volume). The equilibrium melting trend clearly fails to explain the depletion and $\mathrm{Y}$ observed in the boninites as total melting would be required to reduce $\mathrm{Y}$ and $\mathrm{Ti}$ to the abundances observed in the most depleted samples. As Parkinson et al. (this volume) demonstrate using peridotite data, fractional melting (or incremental melting with very small increments) best approximates the melting process. On these diagrams, the fractional melting trend has been plotted together with the composition of pooled melts derived from $10 \%$ melting from mantle that has undergone varying degrees of depletion. On this basis, both diagrams indicate a degree of source depletion in the order of $15 \%-20 \%$. This value, however, is dependent on the distribution coefficients chosen and also on the degree of melting: smaller degrees of melting require less depleted sources. Nonetheless, significant variations in these two parameters are needed to affect greatly the extent of source depletion.

Differences in the $\mathrm{CaO}$ content (for similar wt $\% \mathrm{MgO}$ ) in primitive rocks can give an indication of the fertility or clinopyroxene content of the mantle source since it reflects the ratio of clinopyroxene to olivine + orthopyroxene entering the liquid during partial melting of a lherzolitic source. The low $\mathrm{CaO}$ content of the boninites therefore lends support to a depleted mantle source. Moreover, the presence of very $\mathrm{Cr}$-rich spinels in the boninites (Cr\# 65-84, van der Laan et al., this volume; cf. MORB Cr\# $<60$ ) is further evidence of an origin from a source more refractory than that for MORB. This conclusion agrees with the consensus view (see papers in Crawford, 1989) that boninites are generated from a peridotite source that has already experienced one or more episodes of partial melting. Pearce et al. (this volume) use major element data and arguments based on experimental petrology in an attempt to place further constraints on the degrees of melting involved in boninite genesis at Site 786 and, together with the above results on source depletion, conclude that: (1) the low-Ca boninites were probably derived from a low $(<10 \%)$ degree of melting of the most depleted (cpx-poor lherzolite) mantle source (15\%-20\% depletion of a MORB source), (2) intermediate-Ca boninites were probably derived from higher degrees of melting of a comparable or less depleted source, and (3) the high-Ca boninites were probably produced by higher degrees of melting of the least depleted source $(10 \%-15 \%$ depletion of a MORB source). Note that the trace element data are in broad agreement with these conclusions.

\section{Mantle Source Enrichments}

The general trace element characteristics of the Site 786 volcanics can be illustrated by plotting their compositions normalized to an N-MORB-type composition (Fig. 12). Elements are arranged from right to left on this diagram in order of increasing incompatibility, but with elements mobile in aqueous fluids $(\mathrm{Sr}, \mathrm{K}, \mathrm{Rb}, \mathrm{Ba}$ ) on the left hand side (Pearce, 1983). The Site 786 boninites contain very low abundances of $\mathrm{TiO}_{2}, \mathrm{Y}$, and HREE, compared to N-type MORB, which reflects their origin from a mantle source already depleted by at least one episode of basaltic melt extraction (as discussed above). However, the boninites are variably enriched, relative to these elements, in LIL elements ( $\mathrm{Sr}, \mathrm{Rb}, \mathrm{Ba}$, and Th), LREE's ( $\mathrm{La}$ and $\mathrm{Ce}$ ) and selected HFS elements ( $\mathrm{Zr}$ and $\mathrm{Hf}$ ), and these enrichments are interpreted as one or more components added to the depleted mantle source prior to or during boninite genesis. We now need to investigate the nature and origin of these enriched components.

The most striking feature of the trace-element patterns in Figure 12 is the strong enrichment of LIL elements relative to the REE and HFS elements that affects all of the Site 786 chemical groups. All the volcanics have absolute abundances of the LIL elements which are generally greater than in MORB. The degree of LIL element enrichment can be seen using the Th/Tb vs. Ta/Yb diagram of Pearce (1982) (Fig. 13A). The normalizing factor used (Yb) is effective in largely eliminating variations due to partial melting and fractional crystallization while having minimal participation in the various enrichment processes. Th is used as a representative LIL element because of its relatively immobile behaviour during the alteration of the Site 786 volcanics compared to the other LIL elements. Any melt-related enrichment processes will affect Th and Ta equally because of their similar Kd's during mantle melting, and thus will lie within the "within-plate" trend on Fig. 13A. However, the Site 786 volcanics are all displaced above this "within-plate" trend to higher $\mathrm{Th} / \mathrm{Yb}$. $\mathrm{Th} / \mathrm{Yb}$ is higher in the LCB $(0.3-0.4)$ compared to the HCB and ICB $(0.15-0.4)$. This enrichment of LIL elements has long been recognized as an important feature of subduction-related magmatism (e.g., Gill, 1981; Pearce, 1982). Experimental studies (Tatsumi et al., 1986) have demonstrated the enhanced mobility of LIL elements in hydrous fluids compared with REE and HFS elements, and thus the LIL enrichment is generally attributed to the presence of a fluid component, derived from dehydration of the descending slab, that metasomatized the source (e.g., Gill, 1981; Arculus and Powell, 1986).

The three boninite groups have broadly dish-shaped chondritenormalized REE patterns (Fig. 10), with decreasing abundances from the HREE's to the MREE's (from Lu to Sm), and progressively increasing abundances of the LREE from the MREE's (from Sm to $\mathrm{La}$ ). These patterns are incompatible with either calculated variations in crystal fractionation or degrees of partial melting from a simple spinel lherzolite or harzburgite source. Because the bulk Kd's of the REE's in a spinel lherzolite decrease from $\mathrm{Lu}$ to $\mathrm{La}$, increased degrees of mantle melting or melting of an increasingly more refractory mantle source will result in a lower $\mathrm{La} / \mathrm{Lu}$ ratio in the derived melts. The REE patterns for Hole 786B, however, show the reverse of this: the high-Ca boninites have $\mathrm{La} / \mathrm{Yb}<2$ and the $\mathrm{LCB}$ have $\mathrm{La} / \mathrm{Yb}>2$. This may be the result of either magma mixing, for which no enriched end-member has been found at Site 786, or enrichment in the mantle source prior to or at the onset of melting. Although LREE enrichment in primitive arc-tholeiites has been ascribed to the recycling of pelagic sediment in to the mantle-wedge from the down-going slab (e.g., Hole et al., 1984), Pearce et al. (this volume) reject the involvement of pelagic sediment in the genesis of the Site 786 boninites on the basis of their $\mathrm{Sr}$ and $\mathrm{Pb}$ isotope ratios.

A notable trace element feature of all three boninite groups at Site 786 is their elevated $\mathrm{Zr}$ and $\mathrm{Hf}$ contents relative to $\mathrm{Ti}, \mathrm{Y}$, and M-HREE ( $\mathrm{Sm}$ to $\mathrm{Yb}$ ). The boninites have lower $\mathrm{Sm} / \mathrm{Zr}$ and $\mathrm{Ti} / \mathrm{Zr}$ ratios than MORB. In general, this enrichment is most marked in the LCB group (average $\mathrm{Zr} / \mathrm{Y}=4.7$, average $\mathrm{Ti} / \mathrm{Zr}=41$, average $\mathrm{Zr} / \mathrm{Sm}=43$ ) and least apparent in the late-dike series $\mathrm{HCB}$ group (average $\mathrm{Zr} / \mathrm{Y}=3.5$, average $\mathrm{Ti} / \mathrm{Zr}=57$, average $\mathrm{Zr} / \mathrm{Sm}=40$ ). There is a weak, positive correlation between $\mathrm{Zr} / \mathrm{Yb}$ and $\mathrm{Ta} / \mathrm{Yb}$ for the three boninite groups (Fig. 13B), which suggests that $\mathrm{Ta}$ (and $\mathrm{Nb}$ ) are also carried with the $\mathrm{Zr}$ - and Hf-rich component. Note, however, that $\mathrm{Zr} / \mathrm{Yb}$ and $\mathrm{Ta} / \mathrm{Yb}$ are highly variable in the late dike suite. This may reflect the mobility of $\mathrm{Yb}$ during alteration of these rocks and errors in the determination of $\mathrm{Ta}$ at these low abundances $(0.01-0.05 \mathrm{ppm})$, rather than being a primary magmatic feature. $\mathrm{Ta} / \mathrm{Yb}$ in the boninites is similar to, or less than, N-type MORB, and increases progressively from the late dikes $(0.015-0.04)$, through the intermediate-Ca boninites $(0.035-0.05)$ to 

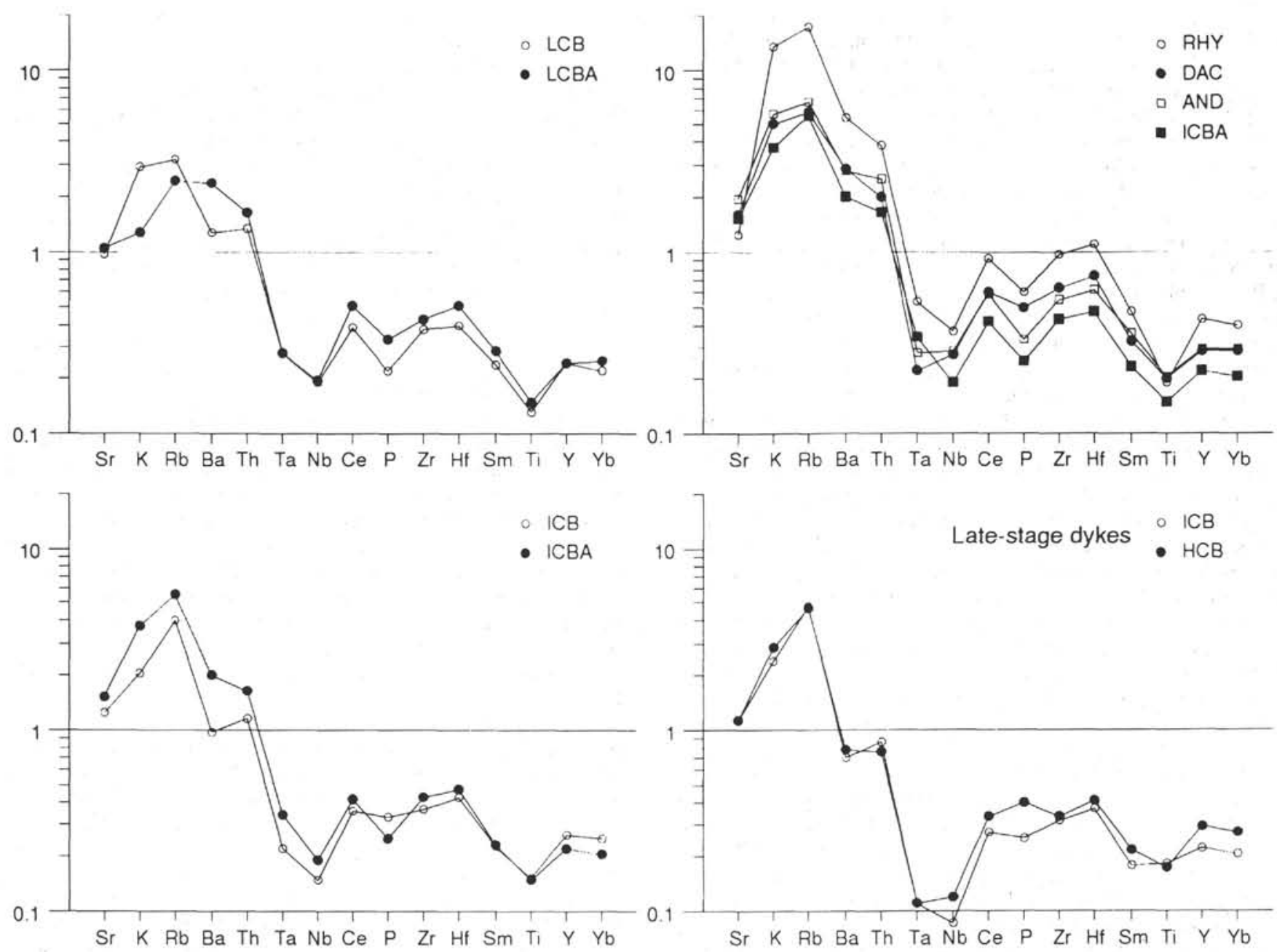

Figure 12. N-type MORB normalized, multi-element patterns (after Pearce, 1983) showing ubiquitous large-ion-lithophile element, light rare-earth element, and $\mathrm{Zr}$ and $\mathrm{Hf}$ enrichment, and low abundance of $\mathrm{Ti}, \mathrm{Y}$, HREE relative to MORB.

the low-Ca boninites $(0.055-0.075)$, despite the major and trace element evidence outlined earlier which suggested that the low-Ca boninite source was more depleted than that of the high-Ca boninites. Thus the origin of the differences in $\mathrm{Ta} / \mathrm{Yb}$ between the three boninite groups may be a complex combination of variable source depletion, differences in the degree of melting, and the addition of a Ta- and $\mathrm{Zr}$-rich component.

Figure 13B shows a plot of $\mathrm{Ta} / \mathrm{Yb}$ vs. $\mathrm{Ce} / \mathrm{Yb}$, on which the Site 786 data trend toward higher $\mathrm{Ta} / \mathrm{Yb}$ with increasing $\mathrm{Ce} / \mathrm{Yb}$. Such a projection has been used by Pearce (1982) to discriminate between hydrous subduction zone components (only affecting $\mathrm{Ce} / \mathrm{Yb}$ ) and silicate melt "within-plate" components, such as ocean island basalts (affecting both $\mathrm{Ta} / \mathrm{Yb}$ and $\mathrm{Ce} / \mathrm{Yb}$ ). The data on Figure 13B clearly have a "within-plate" trend although they are displaced toward higher $\mathrm{Ce} / \mathrm{Yb}$ ratios compared with the mantle array. The relationship between LREE enrichment and $\mathrm{Zr}$, Hf enrichment in the Site 786 boninites can be further assessed using a diagram of $\mathrm{Zr} / \mathrm{Sm}$ vs. $\mathrm{La} / \mathrm{Sm}$ (Fig. 14). The weak, but positive, correlation between $\mathrm{Zr} / \mathrm{Sm}$ and $\mathrm{La} / \mathrm{Sm}$ for the boninites suggests that the enriched component responsible contains both $\mathrm{Zr}$, Hf, and LREE. The $\mathrm{Zr} / \mathrm{Sm}$ ratio in MORB and OIB is 25-28 (Sun and McDonough, 1989) but in the Site 786 boninites, the $\mathrm{Zr} / \mathrm{Sm}$ ratio is $32-70$.

This selective enrichment in $\mathrm{Zr}$ and $\mathrm{Hf}$ has been recognized in many boninite volcanic suites and ophiolitic peridotites (Menzies, 1984; Frey et al., 1985; Frey, 1984; Hickey and Frey, 1982; Coish et al., 1982; Sun and Nesbitt, 1978; Reagan and Meijer, 1984; Cameron, 1985; Hickey-Vargas, 1989; Murton, 1989; Rogers et al., 1989). However, it is not a feature of arc volcanics in general (Pearce, 1983; White and Patchett, 1984). The three main possibilities to develop a LREE-, Zr-rich signature in the Site 786 volcanics, given their forearc setting, are: (1) partial melting processes in the mantle wedge, (2) a subduction component, (3) an asthenospheric component. Although many of the above authors generally attribute the LREE and $\mathrm{Zr}$ enrichments to metasomatism of the lithosphere by the introduction of a LREE, $\mathrm{Zr}$, Hf enriched melt, they do not address the important fractionation of $\mathrm{Zr}$ from $\mathrm{Sm}$. This fractionation is significant since both elements usually exhibit similar petrogenetic behavior (hence their adjacent positions on multi-element patterns: e.g., Pearce, 1983; Sun and McDonough, 1989).

If $\mathrm{Sm} / \mathrm{Zr}$ and $\mathrm{Ti} / \mathrm{Zr}$ are to be fractionated during partial melting, then a mineral phase capable of retaining $\mathrm{Ti}$ and $\mathrm{Sm}$ relative to $\mathrm{Zr}$ must be residual throughout the melting episode. The most promising candidate for this phase is amphibole, as minor phases such as zircon and REE-phases are soluble in basic melts at mantle temperatures (Watson and Harrison, 1984). However this would not be consistent with the scarcity of amphibole in the residual peridotites recovered from the Izu-Bonin forearc during Leg 125 (Ishii et al., this volume).

Unlike the LIL-elements, which are mobile in both hydrous fluids and silicate melts, the HFS-elements are only mobilized by fluorinerich fluids (for which there is no evidence at Site 786) and silicate 

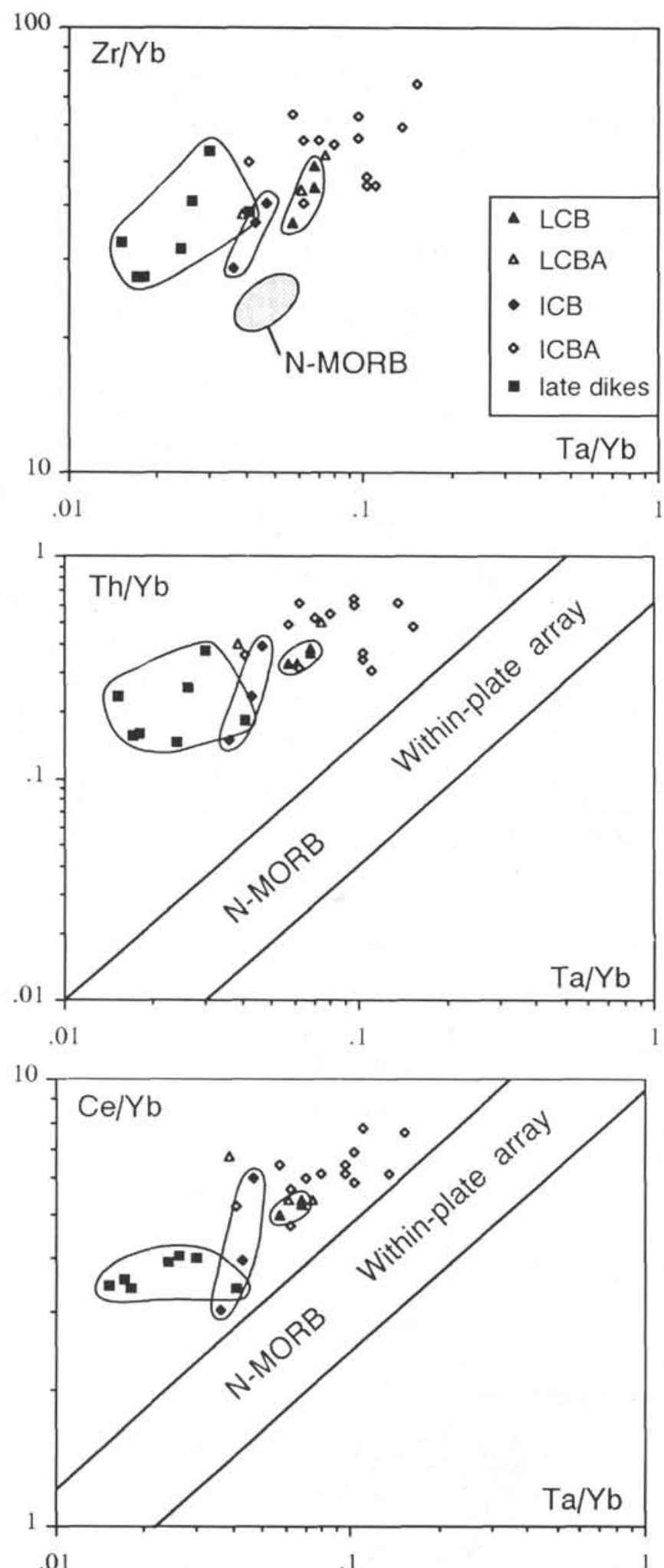

Figure 13. $\mathrm{Ta} / \mathrm{Yb}$ plotted against $\mathrm{Zr} / \mathrm{Yb}, \mathrm{Th} / \mathrm{Yb}$, and $\mathrm{Ce} / \mathrm{Yb}$. Site 786 volcanics all show displacements to high $\mathrm{Th} / \mathrm{Ta}, \mathrm{Zr} / \mathrm{Ta}$, and $\mathrm{Ce} / \mathrm{Ta}$ relative to MORB.

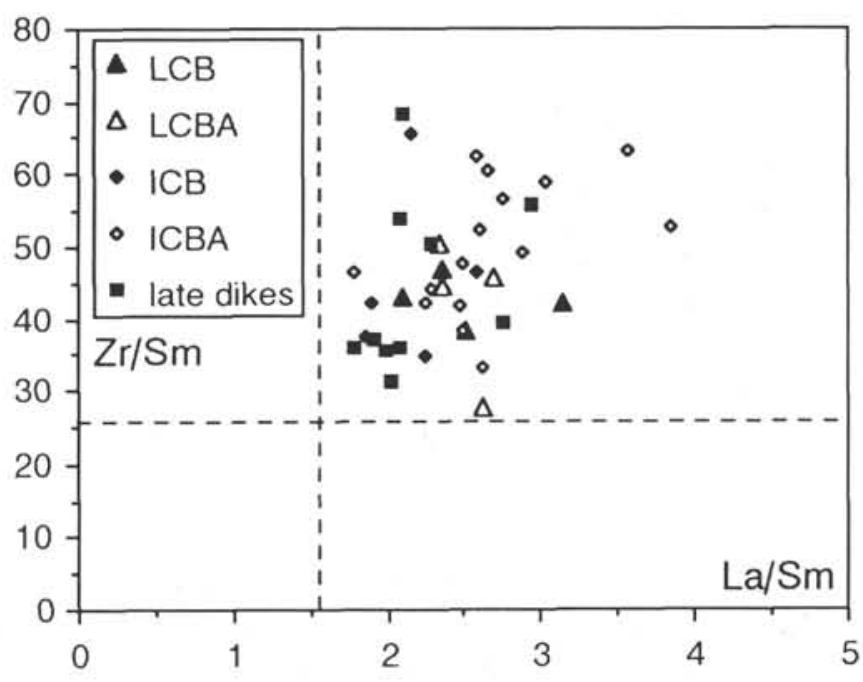

Figure $14 . \mathrm{Zr} / \mathrm{Sm}$ vs. $\mathrm{La} / \mathrm{Sm}$ showing the positive relationship between selective high field strength element enrichment (which is melt controlled) and the light rare earth element enrichment (which can be either melt or volatile fluid controlled). Dashed lines denote chondrite ratios (Sun and McDonough, 1989).

melts (Schneider and Eggler, 1986). Therefore, if the LREE and $\mathrm{Zr}$ enrichment is attributed to a subduction component, then this must be in the form of a silicate melt. However, a total melt from the downgoing oceanic lithospheric slab is precluded as a possible source for the enrichment because ocean floor basalts have near chondritic values for $\mathrm{Zr} / \mathrm{Sm}$ and $\mathrm{Hf} / \mathrm{Sm}$ (White and Patchett, 1984). Moreover, small degrees of melting of a lherzolitic source are unable to fractionate substantially $\mathrm{Zr}$ or $\mathrm{Hf}$ from $\mathrm{Sm}$. Instead, melting of hydrated ocean crust in amphibolite facies, in which amphibole remains in the residue, would release a suitable melt component with elevated $\mathrm{Zr} / \mathrm{Sm}$ and $\mathrm{Hf} / \mathrm{Sm}$ into the overlying mantle wedge. This model is dealt with in more detail in Pearce et al., (this volume). However, Pearce et al., (this volume) show that there is a positive correlation between $\mathrm{Sm} / \mathrm{Zr}$ (or $\mathrm{Ti} / \mathrm{Zr}$ ) and $\mathrm{eNd}$. The HCB and ICB late dikes have higher $\mathrm{Ti} / \mathrm{Zr}$, $\mathrm{Sm} / \mathrm{Zr}$ and $\varepsilon N d(+7.8$ to +7.9$)$ than LCB and ICB of the ca. $41 \mathrm{Ma}$ edifice $(\varepsilon \mathrm{Nd}+6.2$ to +6.8$)$, which indicates that the $\mathrm{Zr}$-rich component has lower $\mathrm{eNd}(<+6)$ than typical MORB. Therefore, instead of melting normal ocean crust, fusion of volcanogenic sediment (derived from OIB-like lavas) or of transitional oceanic crust is required.

A third option for the origin of this enrichment style is by metasomatism of depleted mantle by very small melt fractions from an OIB-like source (Sun and Nesbitt, 1978; Jenner, 1981; Hickey and Frey, 1982; Cameron et al., 1983; Nelson et al., 1984; Murton, 1989; Rogers et al., 1989; Kostopoulos and Murton, in press). Explanations for such a component include: (1) melting of pre-existing mantle heterogeneities (i.e., the plum pudding model of Morris and Hart, 1983); and (2) migration of small-degree melt fractions from the asthenosphere (Frey and Green, 1974; Zindler et al., 1984). Although small degree melt fractions of an OIB-like source would have the requisite isotope characteristics, viz. low $\mathrm{ENd}$, and as well as elevated $\mathrm{Zr} / \mathrm{Yb}$ and $\mathrm{Ta} / \mathrm{Yb}$, they cannot simply explain the unusual $\mathrm{Zr} / \mathrm{Sm}$ fractionation required in the enriched component. One possible solution might come from the interaction between an asthenospheric melt and a mantle wedge containing amphibole-bearing peridotite. The chromatographic effect described by Navon and Stolper (1987), and recently applied to the genesis of boninites by Rogers et al. (1989) and Stern et al. (1991), might allow equilibration of the percolating 


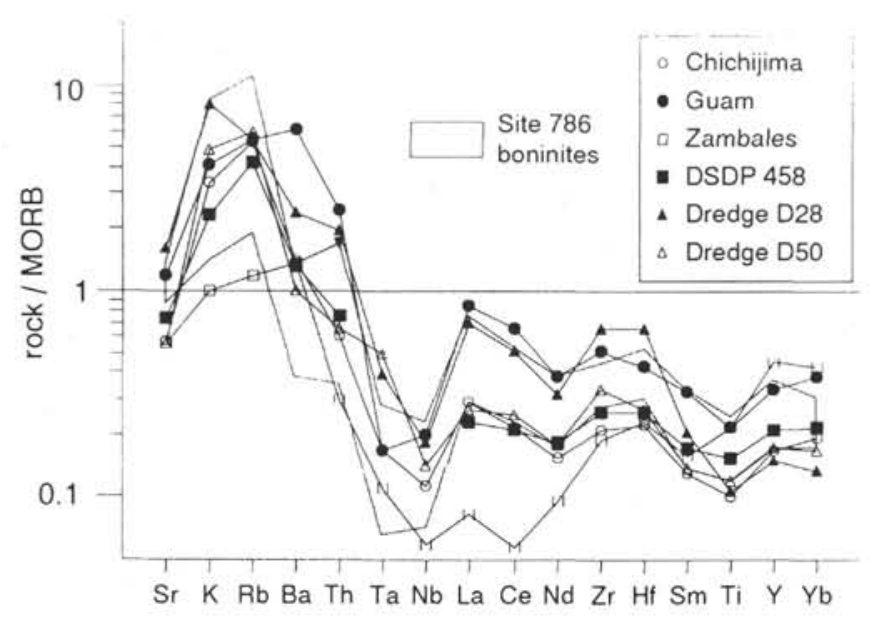

Figure 15. MORB-normalized trace element patterns for samples from other parts of the Western Pacific Eocene boninite province showing the comparison with Site 786 volcanics.

melt with amphibole such that it acquires the high $\mathrm{Zr} / \mathrm{Sm}$ and $\mathrm{Zr} / \mathrm{Ti}$ characteristics. Alternatively, a pre-existing OIB-like enrichment may exist in the mantle wedge. Amphibole is stabilized within the mantle wedge as a result of hydration by fluids released from the subducting slab. Melting of this material, in the presence of residual amphibole, will release the OIB-like component and fractionate $\mathrm{Zr}$ from $\mathrm{Sm}$. These melts could migrate to shallower lithospheric levels and be added to the boninite source region. What is clear from this discussion, therefore, is the problematic nature of the HFS-element enriched component. Only further, detailed studies of boninites will reveal its true nature and provide tighter constraints on its origin.

\section{REGIONAL VARIATIONS}

Eocene-Oligocene boninites and their differentiates have been found throughout the Izu-Bonin-Mariana forearc region over a lateral distance of about $2500 \mathrm{~km}$. Lavas are exposed on the Bonin Islands (Hickey and Frey, 1982; Cameron et al., 1983) and on Guam (HickeyVargas and Reagan, 1987) and Saipan (Meijer, 1983), and boninitic samples have also been dredged from the Mariana inner trench wall (Dietrich et al., 1978; Stern et al., 1991). In addition to ODP Site 7876 in the Izu-Bonin forearc, boninitic and tholeiitic rocks were drilled during DSDP Leg 60 at Site 458 in the Mariana forearc (Wood et al., 1981; Bougault et al., 1981; Sheraskin, 1981; Hickey and Frey, 1981, HickeyVargas, 1989). The Eocene Zambales ophiolite in the Philippines also contains lavas and related plutonic rocks of boninitic affinity (Hawkins and Evans, 1987).

The compositional variability of these lavas (Stern et al., 1991; Pearce et al., this volume) demonstrates that there were considerable differences throughout the Western Pacific region both in the extent of source depletion and in the degree of partial melting involved in boninite generation. Representative trace element analyses for some of these boninite suites are plotted on a MORB-normalized diagram in Figure 15. Like the Site 786 boninites, all of the Western Pacific boninite suites display strong LIL-enrichments that have been interpreted as metasomatism of the sub-forearc mantle by hydrous fluids derived from the subducting Pacific Plate (Wood et al., 1981; Bougault et al., 1981; Sheraskin, 1981; Hickey and Frey, 1981). These boninites have also been variably affected by $\mathrm{Zr}$, Hf enrichment, and some of the regional differences in trace element enrichment are summarized on a $\mathrm{Zr} / \mathrm{Sm}$ vs. $\mathrm{La} / \mathrm{Sm}$ diagram (Fig. 16). Also included on Figure 16 is a field for the active Mariana arc basalts and shoshonites (Lin et al., 1989; Woodhead, 1989). These have chondritic or lower $\mathrm{Zr} / \mathrm{Sm}$ ratios and a wide range of LREE enrichment (La/Yb 1.5-6.5). Samples from Chichijima (Bonin Islands), the

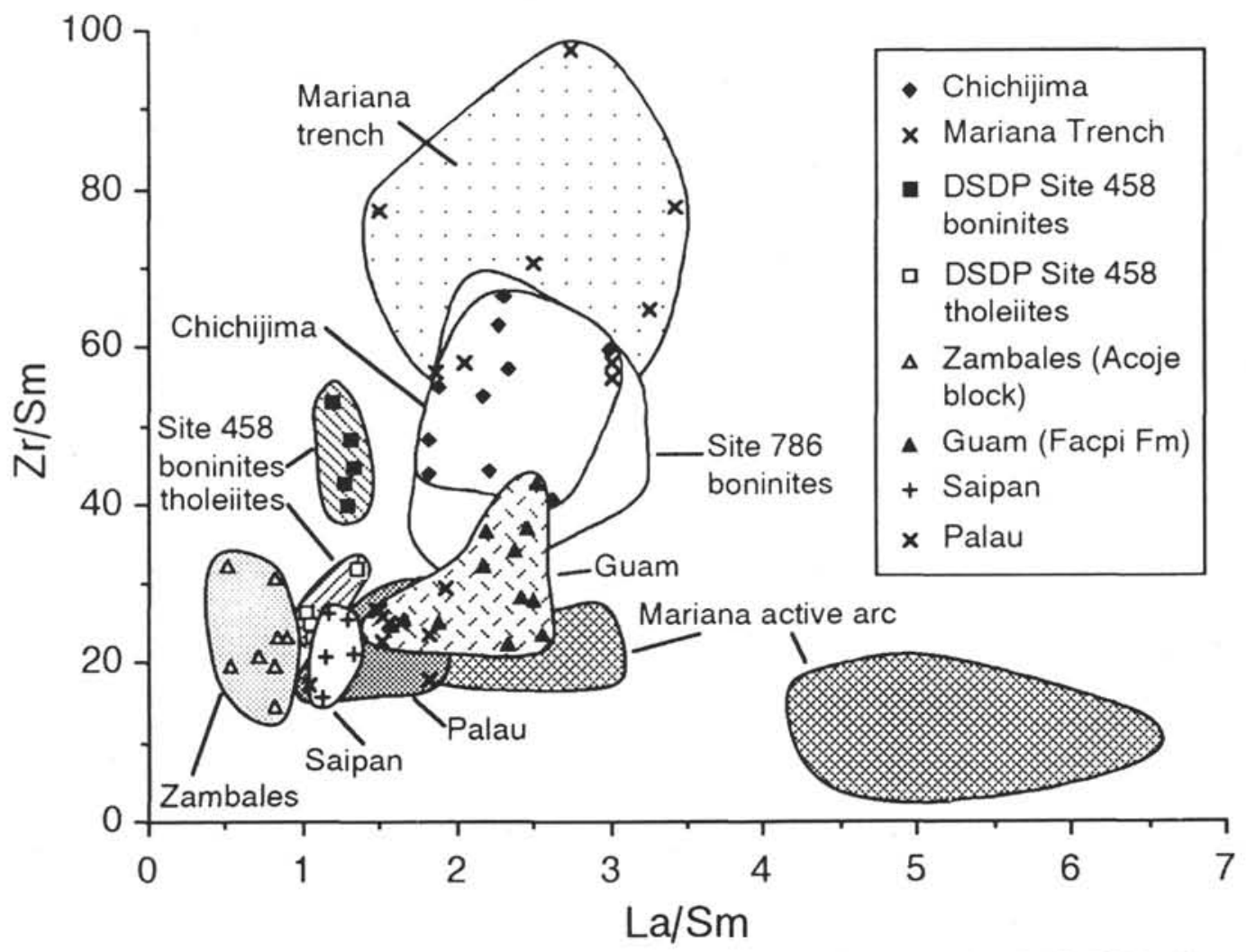

Figure 16. Variations in $\mathrm{Zr} / \mathrm{Sm}$ vs. $\mathrm{La} / \mathrm{Sm}$ for the Western Pacific Eocene boninite suites compared to the Site 786 boninites. 
Mariana Trench dredges and, to a lesser extent, Guam, show a similar enrichment pattern to the Site 786 samples, with LREE-enriched patterns $(\mathrm{La} / \mathrm{Sm}>2)$ and positive $\mathrm{Zr}$ anomalies $(\mathrm{Zr} / \mathrm{Sm}>30)$. Boninites and bronzite-andesites from Site 458 are distinctive, relative to the other Western Pacific boninites, in having elevated $\mathrm{Zr} / \mathrm{Sm}$ (40 to 50 ) but with LREE depleted patterns ( $\mathrm{La} / \mathrm{Sm} \mathrm{1.3-1.4).} \mathrm{In}$ contrast, the Site 458 tholeiitic andesites are similar to boninites from Saipan and Palau in having values of $\mathrm{Zr} / \mathrm{Sm}$ and $\mathrm{La} / \mathrm{Sm}$ similar to or less than chondritic ratios, and plot close to N-Type MORB. The Zambales ophiolite lithologies are strongly LREE depleted $(\mathrm{La} / \mathrm{Sm}<$ 1), but some samples do show a relative enrichment of $\mathrm{Zr}$ and $\mathrm{Hf}$ over $\mathrm{Sm}$ and $\mathrm{Ti}$ (Figure 15).

How is it possible to have sources of different composition, that yield melts simultaneously or within a short time interval within the Izu-Bonin and Mariana forearcs? If we assume that the intraoceanic mantle-wedge beneath both the Izu-Bonin and Mariana forearc regions was produced by spreading at an oceanic ridge, then the lithospheric mantle in the wedge should preserve a vertical compositional zonation, with the most depleted peridotites lying near the top $35 \mathrm{~km}$ ), and mantle fertility increasing with increasing depth (Scott and Stevenson, 1989; Kostopoulos and Murton, in press). Simultaneous melting at different depths within this vertically zoned lithospheric mantle-wedge should yield the type of compositional variation encountered, for example, at Sites 458 and 786. Melting experiments demonstrate that shallow melting $(35 \mathrm{~km})$ of spinelharzburgite under hydrous conditions should yield incompatible trace-element depleted melt compositions (Duncan and Green, 1987; Tatsumi, 1982) similar to the LCB series at Site 786 and the boninites at Site 458 . Hydrous melts from increasing depths in a vertically zoned mantle, could yield a range in melt compositions similar to the variation observed. The Site $786 \mathrm{LCB}$ series and the Site 458 bronzite andesites could originate from the shallowest depths, the parental melts for the Site 786 ICB series originating from intermediate depths, and the parental melts for the Site 458 tholeiitic andesites originating from the greatest depths. We conclude from this that the depth of melting in the Western Pacific forearc lithospheric mantle-wedge spanned a large depth range. The predominance of depleted primary melts in the Izu-Bonin forearc indicates that there was a high geothermal gradient at the onset of subduction; in comparison, the greater range and less depleted primary melt compositions in the Mariana forearc indicates a generally lower geothermal gradient at this time.

Differences in enrichment compositions between Site 458 and Site 786 , are the result of genuine variations either in the type of, or in the transport mechanisms available to, the enriched components. The high $\mathrm{La} / \mathrm{Sm}$ with low $\mathrm{Zr} / \mathrm{Sm}$, characteristic of the Mariana active arc, is evidence of a dominantly fluid controlled process, which also elevated the LIL-elements in these rocks. However, the HFS-element enrichment, which is concomitant with high $\mathrm{La} / \mathrm{Sm}$ at Site 786 , and present to a lesser extent in the Site 458 boninites and bronzite andesites, indicates the predominance of a silicate melt controlled process.

If the HFS-element enrichment is related to a metasomatic event before subduction initiated, then it must have been concentrated within the shallow mantle beneath the Izu-Bonin and Mariana forearc compared to the deeper mantle beneath the active arc. Alternatively, if the HFS-element enriched component originates from partial melts of amphibolitized oceanic basalt during the onset of subduction, then the variations in $\mathrm{Zr} / \mathrm{Sm}$ can be interpreted in terms of the amount of melt from the downgoing slab, the extent of depletion of the source, and the P-T conditions prevailing during subduction. In either case the silicate melt dominated enrichment in much of the Izu-Bonin and Mariana forearcs, indicates a high geothermal gradient for the mantlewedge during the onset of subduction.

\section{SUMMARY}

The Izu-Bonin forearc basement, sampled at Site 786, contains Eocene-age volcanic rocks that include part of the oceanic crust and a volcanic edifice built upon it. It is unlike either the MORB-like Philippine Sea Plate crust (generated at a mid-ocean ridge), or the Mariana active arc (represented by mature arc volcanics), but is instead of boninitic composition.

A remarkably diverse spectrum of rock compositions was recovered at Site 786 , ranging from boninites and bronzite-andesites, through andesites and dacites to rhyolites.Trace element data back-up the classification scheme of Arculus et al. (this volume) for the Site 786 volcanics. The lithological groups can be gathered into three cogenetic series; (1) LCB series (low-Ca boninites and bronzite-andesites), (2) ICB and ADR series (intermediate- $\mathrm{Ca}$ boninites and bronzite-andesites, andesites, dacites, and rhyolites) and, (3) late dike series (high-Ca and intermediate-Ca boninite dikes). Within each series, samples can be related to a distinct parental magma via a complex combination of crystal fractionation and accumulation, although for the late dike series a wider range in parental magmas is involved.

The mobility of many elements during alteration, especially the alkali and alkali-earth elements, $\mathrm{Pb}, \mathrm{U}, \mathrm{REE}$ 's, $\mathrm{Y}$, and $\mathrm{P}$, have conspired to cloud any detailed petrogenetic interpretations. However, the following conclusions have been reached. The boninites have very low abundances of $\mathrm{Ti}$, Y, and HREE relative to MORB, consistent with an origin from a depleted source, and consideration of $\mathrm{Cr}$-Y-Ti melting systematics and major element data indicates that the low-Ca boninites came from a source more depleted than the high-Ca boninite source. The boninites show enrichment in LIL elements, LREE and selected HFS ( $\mathrm{Zr}, \mathrm{Hf})$ relative to $\mathrm{Ti}$, Y, HREE which reflect the addition of a "subduction" component to the boninite source region. The distinctive enrichment of $\mathrm{Zr}$ is a feature not found in typical arc-related volcanics, but it has been recognized in several other boninite suites. The fractionation of $\mathrm{Zr}$ from $\mathrm{Sm}$ and Ti suggests an important role for amphibole in any petrogenetic model to explain the genesis of these boninites. Possibilities include the addition of a melt derived from subducted amphibolitized ocean crust and the integration of an OIB-like melt with amphibole stabilized in the mantle wedge.

The forearc basement was generated from shallow melting of a variably depleted but hydrous mantle-wedge, which was enriched prior to melting by hydrous fluid and silicate melt components of uncertain origin. The presence of HFS-element enriched boninites at Site 786 is evidence of high geothermal gradients within the IzuBonin forearc region during the onset of subduction in the early-middle Eocene. However, the existence of Oligocene-age boninitic volcanism at Site 786 refutes the idea that boninite genesis is exclusive to the onset of subduction.

\section{ACKNOWLEDGMENTS}

The authors wish to thank Sherman Bloomer and Nick Rogers for their thorough reviews of an earlier version of the manuscript, and to Nick Rogers once more, for providing the INAA analyses. Thanks also to Annie Williams and Yvonne Baker for typing, and Dimitri Kostopolous for helpful discussions. B.J.M., D.W.P., and J.A.P. were supported in this work by NERC through an Ocean Drilling Program Special Topics research grant (GR3/416).

\section{REFERENCES}

Arculus, R. J., and Powell, R., 1986. Source component mixing in the region of arc magma generation. J. Geophys. Res., 91:5913-5926. 
Ben-Avraham, Z., and Uyeda, S., 1983. Entrapment of oceanic crust as a backarc basin mode of formation in the western Pacific. In Hilde, T.W.C. (Ed.), Geodynamics of the Western Pacific and Indonesian Region. Int. Geodyn. AGU IGSA Publ., 11:91-104.

Bougault, H., Maury, R. C., Azzouzi, M.E.R., Joron, J.-L., Cotten, J., and Treuil, M., 1982. Tholeiites, basaltic andesites and andesites from Leg 60 sites: geochemistry, mineralogy and low partition coefficient elements. In Hussong, D. M., Uyeda, S., et al., Init. Repts. DSDP, 60: Washington (U.S. Govt. Printing Office), 657-678.

Boynton, W. V., 1984. Cosmochemistry of the rare earth elements; meteorite studies. In Henderson, P. (Ed.), Rare Earth Element Geochemistry: Elsevier (Amsterdam), 63-114.

Cameron, W. E., 1985. Petrology and origin of primitive lavas from The Troodos ophiolite, Cyprus. Contrib. Mineral. Petrol., 89:239-255.

Cameron, W. E., McCulloch, M. T., and Walker, D. A., 1983. Boninite petrogenesis: chemical and $\mathrm{Nd}-\mathrm{Sr}$ isotopic constraints. Earth Planet Sci. Lett., 65:75-89.

Coish, R. A., Hickey, R. L., and Frey, F. A., 1982. Rare earth element geochemistry of the Betts Cove ophiolite, Newfoundland. Geochim. Cosmochim. Acta., 46:2117-2134.

Crawford, A. J., 1989. Boninites and Related Rocks: London (Unwin Hyman),

Dietrich, V., Emmerman, R., Oberhansli, R., and Puchelt, H., 1978 Geochemistry of basaltic and gabbroic rocks from the West Mariana Basin and Mariana Trench. Earth Planet. Sci. Lett., 39:127-144.

Duncan, R. A., and Green, D. H., 1987. The genesis of refractory melts in the formation of the oceanic crust. Contrib. Mineral. Petrol., 96:326-342.

Frey, F. A., 1984. Rare earth element abundances in upper mantle rocks. In Henderson, P. (Ed.), Rare Earth Element Geochemistry: Amsterdam (Elsevier), 153-203.

Frey, F. A., and Green, D. H., 1974. The mineralogy, geochemistry and origin of Lherzolitic inclusions in Victorian basanites. Geochim. Cosmochim. Acta., 38:1023-1059.

Frey, F. A., Suen, C. J., and Stockman, H. W., 1985. The Ronda high temperature peridotite: geochemistry and petrogenesis. Geochim. Cosmochim. Acta., 49:2469-2491.

Gill, J. B., 1981. Orogenic Andesites and Plate Tectonic: New York (Springer Verlag).

Hawkins, J. W., and Evans, C. A., 1983. Geology of the Zambales Range, Luzon, Philippine Islands: ophiolite derived from and island arc- back arc basin pair. In The Tectonic and Geologic Evolution of Southeast Asian Seas and Islands (Pt. 2), Am. Geophys. Union, AGU Monogr. Ser., $27: 95-123$

Hickey, R. L., and Frey, F. A., 1982. Geochemical characteristics of boninite series volcanics: implications for their source. Geochim. Cosmochim. Acta., 46:2099-2115.

, 1982. Rare earth element geochemistry of Mariana forearc volcanics: Deep Sea Drilling Project Sites 458 and Hole 459B. In Hussong, D. M., Uyeda, S., et al., Init. Repts. DSDP, 60: Washington (U.S. Govt. Printing Office), 735-742.

Hickey-Vargas, R., 1989. Boninites and tholeiites from DSDP Site 458, Mariana Forearc. In Crawford, A. J. (Ed.), Boninites and Related Rocks: London (Unwin Hyman), 288-313.

Hole, M. J., Saunders, A. D., Marriner, G. F., and Tarney, J., 1984. Subduction of pelagic sediments: implications for the origin of $\mathrm{Ce}$-anomalous basalts from the Mariana Islands. J. Geol. Soc. London, 141:453-472.

Honza, E., and Tamaki, K., 1985. The Bonin Arc. In Nairn, A.E.M., and Uyeda, S. (Eds.), The Ocean Basins and Margins (Vol. 7): The Pacific Ocean. New York (Plenum), 459-499.

Jenner, G. A., 1981. Geochemistry of high-Mg andesites from Cape Vogel, Papua New Guinea. Chem. Geol., 33:307-332.

Karig, D. E., 1975. Basin genesis in the Philippines Sea. In Karig, D. E., Ingle, J. C., Jr., et al., Init. Repts. DSDP, 31: Washington (U.S. Govt. Printing Office), 857-879.

Kostopoulos, D. K., and Murton, B. J., in press. Origin and distribution of components in Boninite genesis. I. Significance of the OIB component. In Parson, L. M., et al. (Eds.), Ophiolites and Their Modern Oceanic Analogues. Geol. Soc. Spec. Publ. London.

Lin, P.-N., Stern, R. J., and Bloomer, S. H., 1989. Shoshonitic volcanism in the Northern Mariana Arcs. 2. Large-ion lithophile and rare-earth element abundances: evidence for the source of incompatible element enrichments in intraoceanic arcs. J. Geophys. Res., 94:4497-4514.
Meijer, A., 1983. The origin of low-K rhyolites from the Mariana frontal arc. Contrib. Mineral. Petrol., 83:45-51.

Menzies, M. A., 1984. Chemical and isotopic heterogeneities in orogenic and ophiolitic peridotites. In Gass, I. G., et al. (Eds.), Ophiolites and Oceanic Lithosphere. Geol. Soc. Spec. Publ. London, 13:231-240.

Morris, J. D., and Hart, S. R., 1983. Isotopic and incompatible element constraints on the genesis of arc volcanics from Cold Bay and Amak Island, Aleutians, and implications for mantle structure. Geochim. Cosmochim. Acta., 47:2015-2030.

Murton, B. J., 1989. Tectonic controls on boninite genesis. In Saunders, A. D., and Norry, M. J. (Eds.), Magmatism in the Ocean Basins. Geol. Soc. Spec. Publ. London, 42:347-377.

Navon, O., and Stolper, E., 1987. Geochemical consequences of melt percolation: the upper mantle as a chromatographic column. J. Geol., 95:285-307.

Nelson, D. R., Crawford, A. J., and McCulloch, M. T., 1984. Nd-Sr isotope and geochemical systematics in Cambrian boninites and tholeiites from Victoria, Australia. Contrib. Mineral. Petrol., 88:167-172.

Ogawa, Y., and Naka, J., 1984. Emplacement of Ophiolitic rocks in forearc areas: examples from central Japan and Izu-Mariana-Yap island arc systems. In Gass, I. G., Lippard, S. J., and Shelton, A. W. (Eds.), Ophiolites and Oceanic Lithosphere. Geol. Soc. Spec. Publ. London, 13:191-302.

Pearce, J. A., 1975. Basalt geochemistry used to investigate past tectonic environments on Cyprus. Tectonophysics, 25:41-67.

- 1982. Trace element characteristics of lavas from destructive plate boundaries. In Thorpe, R. S. (Ed.), Andesites: New York (Wiley), 525-548.

1983. Role of the sub-continental lithosphere in magma genesis at active continental margins. In Hawkesworth, C. J., and Norry, M. J. (Eds.), Continental Basalts and Mantle Xenoliths: Shiva Press, 230-249.

Pearce, T. H., 1968. A contribution to the theory of variation diagrams. Contrib. Mineral. Petrol., 19:142-157.

Potts, P. J., Thorpe, O. W., Isaacs, M. C., and Wright, D. W., 1985. High precision instrumental neutron-activation analysis of geological samples employing simultaneous counting with both planar and coaxial detectors. Chem. Geol., 48:145-155.

Reagan, M. K., and Meijer, A., 1984. Geology and geochemistry of early arc volcanic rocks from Guam. Geol. Soc. Am. Bull., 95:701-713.

Roeder, P. L., and Emslie, R. F., 1970. Olivine-liquid equilibrium. Contrib. Mineral. Petrol., 29:275-289.

Rogers, N. W., MacLeod, C. J., and Murton, B. J., 1989. Petrogenesis of boninitic lavas from the Limassol Forest Complex, Cyprus. In Crawford, A. J. (Ed.), Boninites and Related Rocks: London (Unwin Hyman), 288-313.

Schneider, M. E., and Eggler, D. H., 1986. Fluids in equilibrium with peridotite minerals: implications for mantle metasomatism. Geochim. Cosmochim. Acta., 50:711-724.

Scott, D. R., and Stevenson, D. J., 1989. A self-consistent model of melting, magma migration and bouyancy driven circulation beneath Mid-Ocean Ridges. J. Geophys. Res., 94:2973-2988.

Sheraskin, A. Y., 1982. Petrology and geochemistry of basement rocks from five Leg 60 sites. In Hussong, D. M., Uyeda, S., et al., Init. Repts. DSDP, 60: Washington (U.S. Govt. Printing Office), 647-656.

Stern, R. J., Morris, J., Bloomer, S. H., and Hawkins, J. W., 1991. The source of the subduction component in convergent margin magmas: trace element and radiogenic isotope evidence from Eocene boninites, Mariana forearc. Geochim. Cosmochim. Acta., 55:1467-1481.

Sun, S.-S., and McDonough, W. F., 1989. Chemical and isotopic systematics of oceanic basalts: implications for mantle composition and processes. In Saunders, A. D., and Norry, M. J. (Eds.), Magmatism in the Ocean Basins. Geol. Soc. Spec. Publ. London, 42:313-345.

Sun, S.-S., and Nesbitt, R. W., 1978. Geochemical regularities and genetic significance of ophiolite basalts. Geology, 6:689-693.

Tatsumi, Y., 1982. Origin of high Magnesian andesites in the Setouchi volcanic belt, southwest Japan, II. Melting phase relations at high pressures. Earth Planet. Sci. Lett., 60:305-317.

Tatsumi, Y., Hamilton, D. L., and Nesbitt, R. W., 1986. Chemical characteristics of fluid phase released from a subducted lithosphere and origin of arc magmas: evidence from high pressure experiments and natural rocks. J. Volcanol. Geotherm. Res., 29:293-309.

Taylor, R. N., Lapierre, H., Vidal, P., Nesbitt, R. W., and Croudace, I. W., in press. Igneous geochemistry and petrogenesis of the Izu-Bonin forearc 
basin. In Taylor, B., Fujioka, K., Janecek, T., et al.,Proc. ODP, Sci. Results, 126: College Station, TX (Ocean Drilling Program).

Watson, E. B., and Harrison, T. M., 1984. Accessory minerals and the geochemical evolution of crustal magmatic systems: a summary and prospectus of experimental approaches. Phys. Earth Planet. Int., 35:19-30.

White, W. M., and Patchett, P. J., 1984. Hf-Nd-Sr isotopes and incompatible element abundances in island arcs: implications for origins and crustmantle evolution. Earth Planet. Sci. Lett., 67:167-185.

Wood, D. A., Marsh, N. G., Tarney, J., Joron, J.-L., Fryer, P., and Treuil, M., 1981. Geochemistry of igneous rocks recovered from a transect across the Mariana Trough, arc, forearc and trench, Site 453 through 461, DSDP Leg 60. In Hussong, D. M., Uyeda, S., et al., Init. Repts. DSDP, 60: Washington (U.S. Govt. Printing Office), 611-646.
Woodhead, J. D., 1989. Geochemistry of the Mariana Arc (western Pacific): source composition and processes. Chem. Geol., 76:1-24.

Zindler, A., Staudigel, H., and Batiza, R., 1984. Isotope and trace element geochemistry of young Pacific seamounts: implications for the scale of upper mantle heterogeneity. Earth Planet. Sci. Lett., 70:175-182.

Date of initial receipt: 25 September 1990

Date of acceptance: 6 November 1991

Ms 125B-133 